\title{
Obscured AGB stars in the Magellanic Clouds
}

\section{IRAS candidates}

\author{
C. Loup ${ }^{1,2}$, A.A. Zijlstra ${ }^{3}$, L.B.F.M. Waters ${ }^{4}$, and M.A.T. Groenewegen ${ }^{5}$ \\ 1 Institut d'Astrophysique de Paris, CNRS, 98 bis Bd. Arago, F-75014 Paris, France \\ 2 European Southern Observatory, Casilla 19001, Santiago 19, Chile \\ 3 European Southern Observatory, Karl-Schwarzschild Strasse 2, D-85748 Garching bei München, Germany \\ 4 Astronomical Institute Anton Pannekoek, University of Amsterdam, Kruislaan 403, NL-1098 SJ Amsterdam, The Netherlands \\ ${ }^{5}$ Max-Planck-Institut für Astrophysik, Karl-Schwarzschild 1, D-85740 Garching, Germany
}

Received March 30, 1996; accepted January 22, 1997

\begin{abstract}
We have selected 198 IRAS sources in the Large Magellanic Cloud, and 11 in the Small Magellanic Cloud, which are the best candidates to be mass-loosing AGB stars (or possibly post-AGB stars). We used the catalogues of Schwering \& Israel (1990) and Reid et al. (1990). They are based on the IRAS pointed observations and have lower detection limits than the Point Source Catalogue. We also made cross-identifications between IRAS sources and optical catalogues.
\end{abstract}

Our resulting catalogue is divided in 7 tables. Table 1 lists optically known red supergiants and AGB stars for which we found an IRAS counterpart ( 7 and 52 stars in the SMC and LMC, respectively). Table 2 lists "obscured" (or "cocoon") AGB stars or late-type supergiants which have been identified as such in previous works through their IRAS counterpart and $J H K L M$ photometry (2 SMC and 34 LMC sources; no optical counterparts). Table 3 lists known planetary nebulae with an IRAS counterpart (4 SMC and 19 LMC PNe). Table 4 lists unidentified IRAS sources that we believe to be good AGB or post-AGB or PNe candidates (11 SMC and 198 LMC sources). Table 5 lists unidentified IRAS sources which could be any type of object (23 SMC and 121 LMC sources). Table 6 lists IRAS sources associated with foreground stars (29 SMC and 135 LMC stars). Table 7 lists ruled out IRAS sources associated with HII regions, hot stars, etc ...

We show that the sample of IRAS AGB stars in the Magellanic Clouds is very incomplete. Only AGB stars more luminous than typically $10^{4} L_{\odot}$ and with a mass-loss rate larger than typically $510^{-6} M_{\odot} /$ yr could be detected by the IRAS satellite. As a consequence, one expects to find very few carbon stars in the IRAS sample. We also expect that most AGB stars with intermediate mass-loss

Send offprint requests to: loup at iap.fr rates have not been discovered yet, neither in optical surveys, nor in the IRAS survey. ${ }^{1}$

Key words: circumstellar matter - stars: latetype - stars: mass-loss — stars: AGB and post-AGB supergiants

\section{Introduction}

During the past 10 years, the observations of the IRAS satellite (Neugebauer et al. 1984) have led to the discovery of a few thousand mass-loosing stars on the Asymptotic Giant Branch (AGB). Some of them are heavily "obscured", in the sense that they lose mass at such a rate $\left(\sim 10^{-5} M_{\odot} /\right.$ yr or more) that their circumstellar envelope becomes optically thick to the stellar radiation. IRAS observations, as well as observations of the millimeter lines of $\mathrm{CO}$ and of the $\mathrm{OH}$ maser emission, have considerably improved our knowledge on their mass-loss rates. However, further studies are severely hampered by the lack of knowledge of the distances, and hence of the luminosities.

The Magellanic Clouds have reasonably well known distances, and they are far enough to consider that all the stars belonging to the same galaxy are at the same distance, with a small uncertainty. In the optical and NIR range, a huge amount of work has been performed in the Small and Large Magellanic Clouds (SMC and LMC) to search for M supergiants and AGB stars. In the LMC, the most complete works, spatially speaking, have been performed by Westerlund and co-workers (Westerlund 1960, 1961; Westerlund et al. 1978; Westerlund et al. 1981),

\footnotetext{
1 Tables 1 to 8 are also available in electronic form at the CDS via anonymous ftp to cdsarc.u-strasbg.fr (130.79.128.5) or via http://cdsweb.u-strasbg.fr/Abstract.html
} 
Sanduleak \& Philip (1977), and Rebeirot et al. (1983), leading to the discovery of several hundred $M$ stars and a few hundred $\mathrm{C}$ stars. These surveys were, however, limited in sensitivity and could only detect the brightest stars $(I<13.5)$. A deeper survey $(I<17)$, but spatially limited, has been performed by Blanco and co-workers (Blanco et al. 1980; Blanco \& McCarthy 1983; Frogel \& Blanco 1990). The SMC has been less studied. The work of Blanco et al. (1980) was the first objective prism survey of this galaxy. This, and the subsequent work of Blanco \& McCarthy (1983) turned up a few hundred carbon and M-type stars. Reid \& Mould (1990) selected AGB star candidates from $V$ and $I$-band photometry in a $0.8^{\circ 2}$ area. Recent works concentrated mainly on carbon stars (Westerlund et al. 1986; Rebeirot et al. 1993) and lead to the discovery of about 2000 of them.

Complementary to previous surveys, people started to search for long-period variables (LPVs) through $I J H K(L)$ photometry (see e.g. Feast et al. 1980; Glass \& Lloyds Evans 1981; Glass \& Feast 1982; Wood et al. 1983; Wood et al. 1985; Glass \& Reid 1985; Reid et al. 1988; Hughes 1989; Feast et al. 1989; Hughes \& Wood 1990). There are now about 1000 LPVs known in the LMC. The most important result of these works is certainly the relations between the luminosity and the period.

As previous studies were based on optical or nearinfrared observations, the resulting samples of stars do not contain optically very thick sources which would be hardly detectable at such wavelengths. In the following we will distinguish between optically identified stars, i.e. stars with optically thin dust shells (typically $J-K>2.5$ ) easily detectable in the optical range, and "obscured" stars, i.e. stars with optically thick dust shells (typically $J-K>2.5$ ) and without optical counterpart. Note that such a separation between optical and obscured stars is partly arbitrary as the transition between optically thin and thick dust shells is of course continuous. The separation is more historical as, complementary to optical surveys, people started to search for AGB stars with high mass-loss rates selecting candidates in the IRAS survey, and confirming (or not) the nature of the selected IRAS sources through $J H K L M$ photometry.

In 1986, Elias et al. selected IRAS sources from the Point Source Catalogue (PSC) with a $12 \mu \mathrm{m}$ flux density, $S_{12}$, larger than $2 \mathrm{Jy}$, and among them discovered two supergiants similar to Galactic OH/IR stars, PSC 04553 - 6825 and PSC 05346 - 6949. The same year, Wood et al. selected IRAS-PSC sources with $S_{25} \gtrsim 0.7 \mathrm{Jy}$ and a $S_{25} / S_{12}$ ratio similar to those of Galactic $\mathrm{OH} / \mathrm{IR}$ stars. They detected the maser emission of $\mathrm{OH}$ in PSC $04553-6825$. This star has an optical counterpart with spectral type M7.5 (Elias et al. 1986). Its optical counterpart was in fact previously known, one can find it as number 64 in Table II of Westerlund et al. (1981). Wood et al. (1992) extended the previous study and detected $\mathrm{OH}$ emission in 5 IRAS-PSC sources. They also deter- mined the period of 9 objects in the LMC. In total, they present a list of $3 \mathrm{SMC}$ and 16 LMC sources that they believe to be late-type stars with thick dust shells. However, in Sect. 4.2. we show that, among these 19 sources, only 9 are actually good candidates being obscured AGB stars or late-type supergiants, the others beeing associated with optically known M supergiants, or with blue supergiants, or even with an HII region or a galaxy. The work by Whitelock et al. (1989) in the SMC is also based on the Point Source Catalogue. They monitored in the $J H K(L)$ bands 5 sources with $S_{25} / S_{12}$ ratios corresponding to a colour temperature of a few $100 \mathrm{~K}$. Among these 5 sources, 2 are long-period variables without optical counterparts, 1 is associated with an M star, 1 is a peculiar carbon star, and 1 is associated with a blue supergiant.

In addition to the survey observations, IRAS also made pointed observations, with orthogonal scan directions, notably in the direction of the Magellanic Clouds. The corresponding detection limits are fainter than those of the PSC. The IRAS pointed observations cover the major part of the SMC and the LMC, except the outer regions. These data have been reduced and published in a catalogue by Schwering \& Israel (1990). Part of these data in the LMC has also been reduced by Reid et al. (1990) with the aim of searching for obscured AGB stars. They also made photographic I plates and give possible optical counterparts of some IRAS sources. With additional JHK observations, Reid (1981) discovered 10 "cocoon" stars, i.e. AGB stars with optically thick dust shells, associated with IRAS sources. He also showed that, for these "cocoon" stars, the optical counterpart proposed by Reid et al. was in fact not associated with the IRAS source as he found a much redder object close to the IRAS position. More recently, based on the source selections presented here, Zijlstra et al. (1996), in the second paper of this series (hereafter called Paper II), identified 16 additional AGB stars with optically thick dust shells and estimated their mass-loss rates.

In this paper, we will first adress the question: "What are the properties of AGB stars detected by IRAS in the LMC?" (Sect. 2). In Sect. 3, we present our selection of IRAS sources, from the catalogues of Schwering \& Israel (1990) and Reid et al. (1990) in order to find AGB stars candidates. We systematically searched for optical identifications of all the selected IRAS sources. In Sect. 4 we present final tables, optical stars with an IRAS counterpart in Table 1, obscured AGB stars or supergiants without optical counterpart in Table 2, planetary nebulae in Table 3, unidentified IRAS sources that we think to be good obscured AGB (or post-AGB) stars candidates in Table 4, and foreground stars in Table 6 . In Sect. 5 we discuss on the reliability of IRAS observations at flux levels close to the detection limits and compare both catalogues. 


\section{What are the properties of AGB stars detected by IRAS in the LMC?}

Previous works by Elias et al. (1986), Wood et al. (1986, 1992), Reid et al. (1990), and Reid (1991) have shown that some red supergiants and AGB stars have been detected by IRAS in the LMC, at least at $12 \mu \mathrm{m}$. As the distance of the LMC is about $50 \mathrm{kpc}$, one may however be surprised that IRAS could detect AGB stars so far away. A comparison with stars of our Galaxy could allow us to answer this question and to define the physical properties of such stars. Reid et al. give part of the answer based on the most optically thick OH/IR stars known in the Galaxy, and on the "prototype" of optically thick carbon stars, IRC +10216 . They conclude that AGB stars with similar physical properties should "be detected with ease" in the IRAS survey.

Lets consider as an example one of the most extreme carbon star AFGL 3068 (Price \& Walker 1976), and the well known O-rich star WX Psc. AFGL 3068 is particularly optically thick as $[K-L]=7$ (le Bertre 1992). WX Psc has a known optical counterpart but is not optically thin as the $10 \mu \mathrm{m}$ silicate feature is slightly self-absorbed. Assuming an intrinsic luminosity of $10^{4} L_{\odot}$, their distances would be 0.95 and $0.54 \mathrm{kpc}$ respectively (see e.g. Loup et al. 1993). At $50 \mathrm{kpc}$, such stars would have $12 \mu \mathrm{m}$ IRAS flux densities of 0.25 and $0.13 \mathrm{Jy}$, and $25 \mu \mathrm{m}$ IRAS flux densities of 0.28 and $0.11 \mathrm{Jy}$, respectively. The sensitivity limit of the IRAS-PSC is $0.25 \mathrm{Jy}$ at 12 and $25 \mu \mathrm{m}$, and 0.15 and $0.22 \mathrm{Jy}$ in the catalogue of Schwering \& Israel (1990). Therefore a source like AFGL 3068 could be detected in the LMC, but not easily, and WX Psc would not be detected (or by chance as Schwering \& Israel report a few 12 and $25 \mu \mathrm{m}$ detections at a level of $0.07 \mathrm{Jy}$ ). The intrinsic luminosity of these 2 stars could be larger than $10^{4} L_{\odot}$, but also smaller, and in addition their luminosity varies by more than a factor 2 .

In an attempt to get a more complete overview, we have used the sample of Galactic sources whose CO emission in the rotational transitions $J=1-0$ or/and $J=2-1$ has been detected (Loup et al. 1993). Though this sample is strongly observationally biased, it contains all the chemical types (O-rich, C-rich, and S stars), and covers the whole range of mass loss rates $\left(10^{-7}\right.$ to $\left.10^{-4} M_{\odot} \mathrm{yr}^{-1}\right)$. This sample contains about 400 AGB stars and a few M supergiants. Their bolometric luminosities have been calculated from optical, JHKL $(M)$, and IRAS photometry, when enough data were available; ortherwise they were estimated using the bolometric correction to IRAS data of van der Veen \& Rugers (1989). Distances have been estimated assuming an intrinsic luminosity of $10^{4} L_{\odot}$ for the AGB stars, and $10^{5} L_{\odot}$ for the supergiants, corresponding to bolometric luminosities of -5.25 and -7.75 , respectively.

Figure 1 shows their IRAS color $C_{21}=\log$ $\left(12 S_{25} / 25 S_{12}\right)$ as a function of their $12 \mu \mathrm{m}$ IRAS flux density scaled to $50 \mathrm{kpc}$. The dashed lines indicate the IRAS-PSC and Schwering \& Israel sensitivity limits, 0.25 and $0.15 \mathrm{Jy}$. Note that the faintest sources at $12 \mu \mathrm{m}$ in Schwering \& Israel have $S_{12}=0.07 \mathrm{Jy}$. The two correlations appearing in Fig. 1 for O-rich and C-rich stars only reflect the bolometric correction of van der Veen \& Rugers (1989) and are not real. It appears clearly that, whatever the value of $C_{21}$, very few AGB stars could be detected in the PSC if they are not more luminous than $10^{4} L_{\odot}$. The situation is a little better with the IRAS pointed observations, but we still expect that most AGB stars with $L \leq 10^{4} L_{\odot}$ have not been detected by IRAS. The faintest "obscured" AGB star discovered until now actually has a bolometric luminosity of $-5.1\left(8700 L_{\odot}\right)$, and a $12 \mu \mathrm{m}$ flux density of $0.13 \mathrm{Jy}$ (Reid 1991; Reid et al. 1990). The IRAS color $C_{21}$ can be considered as a rough estimator of the total dust opacity in the circumstellar shell, and hence as a rough estimator of the mass-loss rate (see e.g. RowanRobinson et al. 1986; Bedijn 1987; Chan \& Kwok 1990). As expected, one sees in Fig. 1 that only optically thick AGB stars could be detected by IRAS as the value of $S_{12}$ decreases drastically when $C_{21}$ decreases. Comparing Fig. 1 with Fig. 9b in Loup et al. (1993), we conclude that most AGB stars detected by IRAS in the LMC should have a mass-loss rate larger than $510^{-6} M_{\odot} \mathrm{yr}^{-1}$. Sources with intermediate mass-loss rates are probably still almost totally undiscovered in the MCs as they would already be too faint for optical surveys, but not optically thick enough to have been seen by IRAS. This is very well illustrated for carbon stars in the Fig. C1 of Groenewegen \& de Jong (1993) where they show the theoretical relation between $S_{12}$ and the $I$ magnitude for various bolometric luminosities and mass-loss rates.

The sample of sources detected in CO (Loup et al. 1993) contains only a few supergiants, which does not allow a statistical overview. We consider the example of $\alpha$ Ori, VY CMa, and VX Sgr. If their luminosity is $10^{5} L_{\odot}$, their mass-loss rates estimated from $\mathrm{CO}$ observations are $\sim 10^{-6}, 410^{-6}$, and $510^{-6} M_{\odot} \mathrm{yr}^{-1}$, respectively. Their $12 \mu \mathrm{m}$ IRAS flux densities scaled to $50 \mathrm{kpc}$ are $0.07,1.7$, and 0.18 Jy. So even LMC red supergiants should have a relatively optically thick dust envelope to be detected by IRAS.

From the previous considerations we expect the IRAS sample of LMC AGB stars to be very incomplete, strongly biased towards luminous (more than $10^{4} L_{\odot}$ ) and optically thick sources (without optical counterpart; mass-loss rate larger than $\left.510^{-6} M_{\odot} \mathrm{yr}^{-1}\right)$. Most AGB stars with a bolometric luminosity fainter than about -5.2 , even if very optically thick, have probably not been detected by IRAS. In particular, we therefore expect to find far fewer C-rich stars than O-rich stars in the IRAS sample, though carbon stars are more numerous than late $\mathrm{M}$ stars in the MCs (Blanco et al. 1980). Groenewegen \& de Jong (1993) reach the same conclusion through a theoretical approach. 


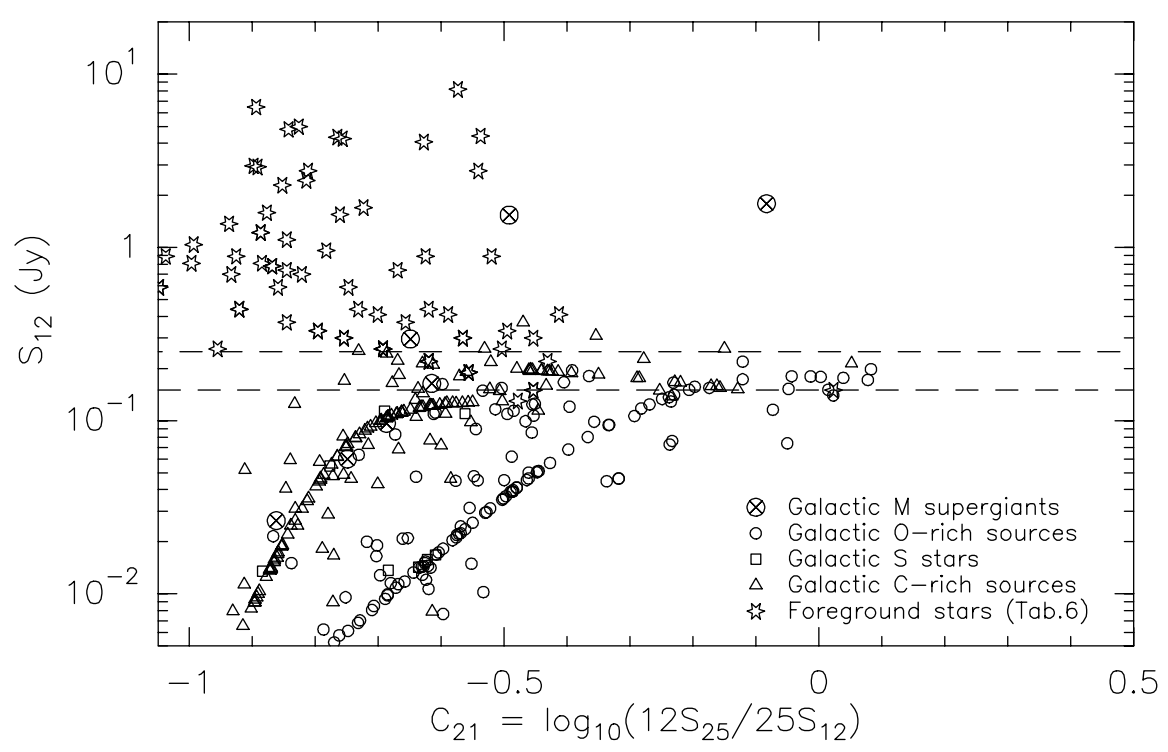

Fig. 1. Location of galactic AGB stars and M supergiants detected in the CO $(1-0)$ or CO (2-1) lines (Loup et al. 1993) in a $\left[C_{21}, S_{12}\right]$ diagram as if they were located in the LMC. Distances were calculated from bolometric fluxes and assuming a luminosity of $10^{4} L_{\odot}$ for AGB stars, $10^{5} L_{\odot}$ for M supergiants. $S_{12}$ was then scaled to $50 \mathrm{kpc}$. Symbols are defined in the figure. The two correlations seen for $\mathrm{M}$ and $\mathrm{C}$ stars come from the bolometric correction of van der Veen \& Rugers and are not real (see also Sect. 2). The two dashed lines correspond to the detection limit of the PSC (0.25 Jy at $12 \mu \mathrm{m})$ and of Schwering \& Israel $(0.15 \mathrm{Jy})$. Also plotted in Fig. 1 are the foreground stars listed in Table 6. One can see that, for most of them, their location in the diagram would be sufficient to determine that they do not belong to the LMC

\section{Selection criteria}

The location of AGB stars, post-AGB stars, planetary nebulae, HII regions, and galaxies, in the IRAS two-colour diagram (12-25-60) has been described by many authors (see e.g. van der Veen \& Habing 1988). In this paper we use the work by Pottasch et al. (1988, their Fig. 1) which gives an overview. We are not only interested in AGB stars and M supergiants, we also would like to include possible post-AGB objects and planetary nebulae. Therefore the problem is mainly to eliminate HII regions and galaxies. According to Fig. 1 in Pottasch et al., most HII regions have $\left(S_{12} / S_{25}\right)<0.4\left(C_{21}>+0.08\right)$ and $\left(S_{25} / S_{60}\right)<0.3$ $\left(C_{32}=\log \left(25 S_{60} / 60 S_{25}\right)>+0.14\right)$, and most galaxies have $\left(S_{12} / S_{25}\right)<1\left(C_{21}>-0.32\right)$ and $\left(S_{25} / S_{60}\right)<0.3$. The knowledge of the 12, 25, and $60 \mu \mathrm{m}$ IRAS flux densities would allow us to select AGB stars, post-AGB stars and planetary nebulae with great confidence.

The sensitivity limits given by Schwering \& Israel (1990) are 0.15, 0.22, and $0.41 \mathrm{Jy}$ at 12,25 , and $60 \mu \mathrm{m}$ respectively. From the discussion in section 2 , or from the work by Reid (1991), we expect that most AGB stars detected by IRAS in the LMC will not be detected at $60 \mu \mathrm{m}$, and often will be detected only in one band, at 12 or $25 \mu \mathrm{m}$. The selection of sources is then not so straightforward, and we have to use limits on the IRAS flux ratios. In practice, we have to consider two possiblities at $12 \mu \mathrm{m}$ : detected or not detected, and three possibilities at 25,60 , and $100 \mu \mathrm{m}$ : detected, not detected, or contaminated by the surroundings. Non detections provide us upper limits on the IRAS flux densities. Contaminated fluxes do not give us any information.

As a first selection, we have removed all the sources that Schwering \& Israel find extended (23 sources in the LMC, 49 sources in the SMC). Next we have removed all the sources with $S_{100}>2 S_{60}$ (945 sources in the LMC, 58 sources in the SMC); note that this eliminates all the sources detected at $100 \mu \mathrm{m}$ but not detected at $60 \mu \mathrm{m}$. This criterion removes many HII regions and galaxies which are "cold" objects, preferentially detected at long wavelengths. It could, however, also remove some planetary nebulae.

From these two first selections, we obtained a sample of 923 sources in the LMC, and 142 sources in the SMC. We also added 24 LMC sources, with $S_{100}<2 S_{60}$ or not detected at 60 and $100 \mu \mathrm{m}$, listed in Reid et al. (1990) which have not been found by Schwering \& Israel (conversely some sources mentioned in Schwering \& Israel have not been found by Reid et al.). Then we applied the following selection criteria:

$\left(S_{12} / S_{25}\right)>1$, whatever $\left(S_{25} / S_{60}\right)$

or $\left(S_{12} / S_{25}\right)<1$ and $\left(S_{25} / S_{60}\right)>0.3$.

There are several cases where we do not have any information on one of the flux ratios, or where the limit derived on one of the flux ratios is not significant, so we cannot conclude anything. We have divided the initial sample into 
three groups. Group 1 contains 256 LMC and 37 SMC sources following the selection criteria, and expected to be red supergiants, AGB stars, post-AGB stars, or PNe candidates. Group 3 contains 415 LMC and 62 SMC sources which do not follow the selection criteria, expected to be HII regions or galaxies. Group 2 contains 276 LMC and 43 SMC sources for which the available IRAS data are not conclusive, they can be a priori anything.

\section{Description of tables}

The previous selection based only on IRAS data is a first step to get a global list of candidates. However we expect to find in this list many foreground stars as well as some star clusters, and some IRAS candidates could be associated with optically known red supergiants. We also have to remove from this first list IRAS sources which have been identified in previous works as obscured AGB stars or late-type supergiants, through JHKLM photometry. We therefore tried to find counterparts to the IRAS sources of groups 1 and 2. For that purpose we have extensively used the Simbad database, as well as a compilation of most works on AGB stars and M supergiants in the Magellanic Clouds (see references), as some of them have not been entered in the Simbad database (in particular the surveys by Blanco et al. 1980; Westerlund et al. 1978, 1981). Cross-identifications are mainly based on the positions of the objects, but also on the consistency between the optical magnitudes and the IRAS fluxes and colours. For IRAS sources already listed in the PSC, we have searched around $30^{\prime \prime}$ from the IRASPSC position; for new IRAS sources not discovered in the $\mathrm{PSC}$ we have searched around $60^{\prime \prime}$ as the position given by Schwering \& Israel is not more accurate. The IRAS-PSC uncertainty is in principle smaller than $30^{\prime \prime}$. However, as the IRAS flux densities of our sources are often close to the sensitivity limit, the position might be less accurate than usual. In addition, we notice that positions given by Schwering \& Israel and Reid et al. sometimes disagree by more than $60^{\prime \prime}$. On the other hand, the JHKL counterpart of obscured AGB stars is generally close (within $20^{\prime \prime}$ ) to the IRAS-PSC position (see Reid 1991; Zijlstra et al. 1996, Paper II).

Finally, we have divided the IRAS sources into 7 tables which will be described below:

\subsection{Tables 1, 2, and 3: identified objects}

Optically known red supergiants and AGB stars are listed in Table 1, obscured AGB stars and late-type supergiants without optical counterparts in Table 2, and planetary nebulae in Table 3. Column 1 lists the LI number as given in Schwering \& Israel, and Col. 2 the TRM number as given in Reid et al. A " $\star$ " in front of the LI number means that the IRAS source was also found in the PSC. Columns 3 and 4 list the coordinates as found in Schwering
\& Israel or in Reid et al. (when the TRM source has not been found by Schwering \& Israel); note that Schwering $\&$ Israel provide coordinates more accurate than $60^{\prime \prime}$ only when the source is also in the PSC, and then they just give the IRAS-PSC coordinates. Columns 5, 6, 7, and 8 list the IRAS flux densities at 12, 25, 60, and $100 \mu \mathrm{m}$, respectively, as found in Schwering \& Israel (or in Reid et al.); "C" means contaminated, and ":" means that the value is uncertain. Column 9 and 10 list the IRAS colours, $C_{21}=\log \left(12 S_{25} / 25 S_{12}\right)$ and $C_{32}=\log \left(25 S_{60} / 60 S_{25}\right)$. Column 11 gives the group (1,2 or 3$)$ of the IRAS source as defined in Sect. 3. When we give two possibilities for the group, the second value corresponds to the group found by using the Reid et al.'s values if the group is different from the one derived from the Schwering \& Israel values (see also Table 8). The last column gives the identification: source name, some observational information (between bracket), and a code for references (between square brackets). The list of references is given at the end of the tables, as well as, for Table 1, an overview of the various optical identifications. Table 1 lists $52 \mathrm{LMC}$ and 7 SMC optical stars. Table 2 contains 34 LMC and 2 SMC sources. Among the 34 LMC obscured AGB stars (or late-type supergiants), 16 have been identified recently by Zijlstra et al. (1996, Paper II) on the basis of the present work (for selection) and infrared observations in the $J H K L^{\prime}$ bands and at $10 \mu \mathrm{m}$.

The reader will notice that Table 1 contains 2 sources known to be optically thick, in particular the famous PSC 04553 - 6825 (LI-LMC 181) discovered by Elias et al. (1986) and Wood et al. (1986). This source has an optical counterpart (WOH G064 in Westerlund et al. 1981, spectral type M7.5 in Elias et al. 1986) and is therefore listed in Table 1. PSC 00350-7436 (LI-SMC 5) has an optically thick dust shell as well. As Whitelock et al. (1989) could determine its spectral type, a peculiar carbon star, we list it in Table 1. Other sources in Table 1 do not have optically very thick dust envelopes.

\subsection{Comments on the Wood et al.'s (1992) list}

Wood et al. monitored in $J H K L 3$ sources in the SMC and 16 in the LMC. The results are presented in their Tables 3 and 5. Among the $16 \mathrm{LMC}$ sources, we found that PSC 04553 - 6825 (LI-LMC 181), possibly PSC 05247 - 6941 (LI-LMC 976), PSC 05261 - 6614 (LI-LMC 1033), and PSC 05389 - 6922 (LI-LMC 1470), have a known optical counterpart of spectral type M: WOH G064, WOH SG264, WOH SG281, and WOH SG455, respectively (Westerlund et al. 1981, see Table 1 caption). They are listed here in Table 1. PSC 04571-6954 (LI-LMC 225) can be identified with the well know S Dor variable HD 268835 of spectral type B8Ia and is listed in Table 7. PSC 05244-6832 (LILMC 961) and PSC 05325 - 6743 (LI-LMC 1274) have extremely red IRAS colours and fall in our group 3 . They can be identified with the HII regions LHA $120-\mathrm{N} 138 \mathrm{D}$ 
and LHA 120-N 57A, respectively (Henize 1956), and are listed in Table 7 . The 3 SMC sources all have very red IRAS colours and fall in group 3 too. There is a probable M supergiant close to PSC 00477 - 7343 (LI-SMC 57), so we list this source in Table 1 . However we think that the association between the IRAS source and the optical star is doubtful. PSC $00521-7054$ is identified with a galaxy and is listed in Table 7. Finally, for PSC 01039-7305 (LISMC 173) we could not find any identification. However, in addition to its unusual IRAS colours, it also has unusual $J H K L$ colours for an AGB star, so we again list it in Table 7.

Wood et al. also list 14 sources in their Table 4 that they could detect in $J H K$. Among them, PSC $05027-$ 7124 (LI-LMC 346), PSC 05198 - 6941 (LI-LMC 816), and PSC 05280 - 6910 (LI-LMC 1100), belong to groups 1 or 2 . They are however in Table 7 as associated with the blue supergiant HD 269006, two hot stars, and NGC 1984, respectively. The other objects all belong to group 3 and have very red IRAS colors. We list all of them in Table 7, either because they are associated with hot stars, or with HII regions, or because the NIR colors are much too blue compared to the IRAS colors. We do not exclude that the NIR colors actually correspond to an AGB star, however the association with the IRAS source is unlikely.

\subsection{Tables 4 and 5: unidentified IRAS sources}

Unidentified IRAS sources from group 1 are listed in Table 4 (AGB stars, post-AGB stars, and PNe candidates), and those from group 2 in Table 5 (IRAS data insufficient to conclude on their nature). Columns 1 to 10 are the same as in Tables 1, 2, 3. For some sources we found one or several objects close to the IRAS position, but we think that the association with the IRAS source is unlikely. In particular, we found some IRAS sources close to optical C stars or LPVs, but the IRAS flux densities are much too bright compared to what one expects from the optical and JHKL properties of the stars. Such cases are listed at the end of the Tables. Table 4 contains 198 LMC and 11 SMC sources, so about 6 times more new obscured AGB stars candidates than what is already known as listed in Table 2.

\subsection{Table 6: foreground stars}

It contains all the sources from groups 1 and 2 found or believed to be foreground stars. The columns are the same as in Tables 1, 2, and 3. Identifications of foreground stars are based on the $V$ (or $I$ ) magnitude of the star, and/or its spectral type, and/or its heliocentric velocity. Most of them have M, K, F, G giant or dwarf types. For three sources in the LMC, we did not find any optical star at the location of the IRAS source. However, their location in Fig. 1 shows that they are very likely foreground stars.
Table 6 contains 135 and 29 stars in the fields of the LMC and the SMC, respectively.

\subsection{Table \%: ruled out objects}

It contains all the sources, from groups 1 and 2 , that we have ruled out after the selection described in Sect. 3 . They are mostly associated with star clusters, or/and hot stars, Wolf-Rayet stars, or blue luminous variables; there are also a few HII regions and galaxies. Table 7 contains 76 LMC and 15 SMC sources, including the group 3 sources from Wood et al. (1992, see Sect. 4.2).

\section{Discussion}

Despite the selection criteria we use, there are 6 stars of group 3 in Table 1, and 3 in Table 2 . All these sources have $S_{100}$ larger than $S_{60}$, or a very large $S_{60} / S_{25}$ ratio, which is normally characteristic of HII regions and galaxies. A look at the IRAS maps show that these sources are located in regions with a high background at 100 and $60 \mu \mathrm{m}$, and that $S_{100}$ and/or $S_{60}$ are contaminated. Three sources, LI-LMC 932, 1107, and 528, are also found by Reid et al. (1990). They do not give any $100 \mu \mathrm{m}$ flux, and find no or a fainter $60 \mu \mathrm{m}$ flux. Using the fluxes determined by Reid et al., these sources would belong to group 1 or 2 (see Table 8). We expect that probably a few good AGB candidates have been ruled out of our list for similar reasons, rejected because of a high 100 or/and $60 \mu \mathrm{m}$ flux due to contamination. There is however no systematic way to pick up such sources, the only way would be a careful examination of IRAS maps for all of them. The case of planetary nebulae is more marginal. In Table 3, 9 sources belong to group 3 . However, they are normally very cold objects and their IRAS flux ratios are close to the limits of our selection criteria.

Conversely there are several rejected sources in Table 7 belonging to group 1. Most of them are, however, associated with star clusters, or hot stars, which is not contradictory with belonging to group 1 . There are a few very interesting sources, blue luminous variables and Wolf-Rayet stars which deserve more studies. The few sources of group 1 associated with HII regions all have very cold IRAS colors, close to the limits of our selection criteria.

In Fig. 2, we have plotted the location of optically known stars (Table 1, Fig. 2a), obscured AGB stars (Table 2, Fig. 2b), and planetary nebulae (Table 3, Fig. 2c), in the same kind of diagram as the one presented in Fig. 1 for galactic sources and foreground stars (Table 6). One would expect that optical stars have $C_{21}$ smaller than $-0.3\left(S_{12} \sim S_{25}\right)$, however in Fig. 2a $28 \%$ of the sources have $C_{21}>-0.3$. Most stars in Table 1 are $M$ supergiants. In our Galaxy, it is known that some M supergiants have a "cold" circumstellar envelope though not optically thick. To model their energy 

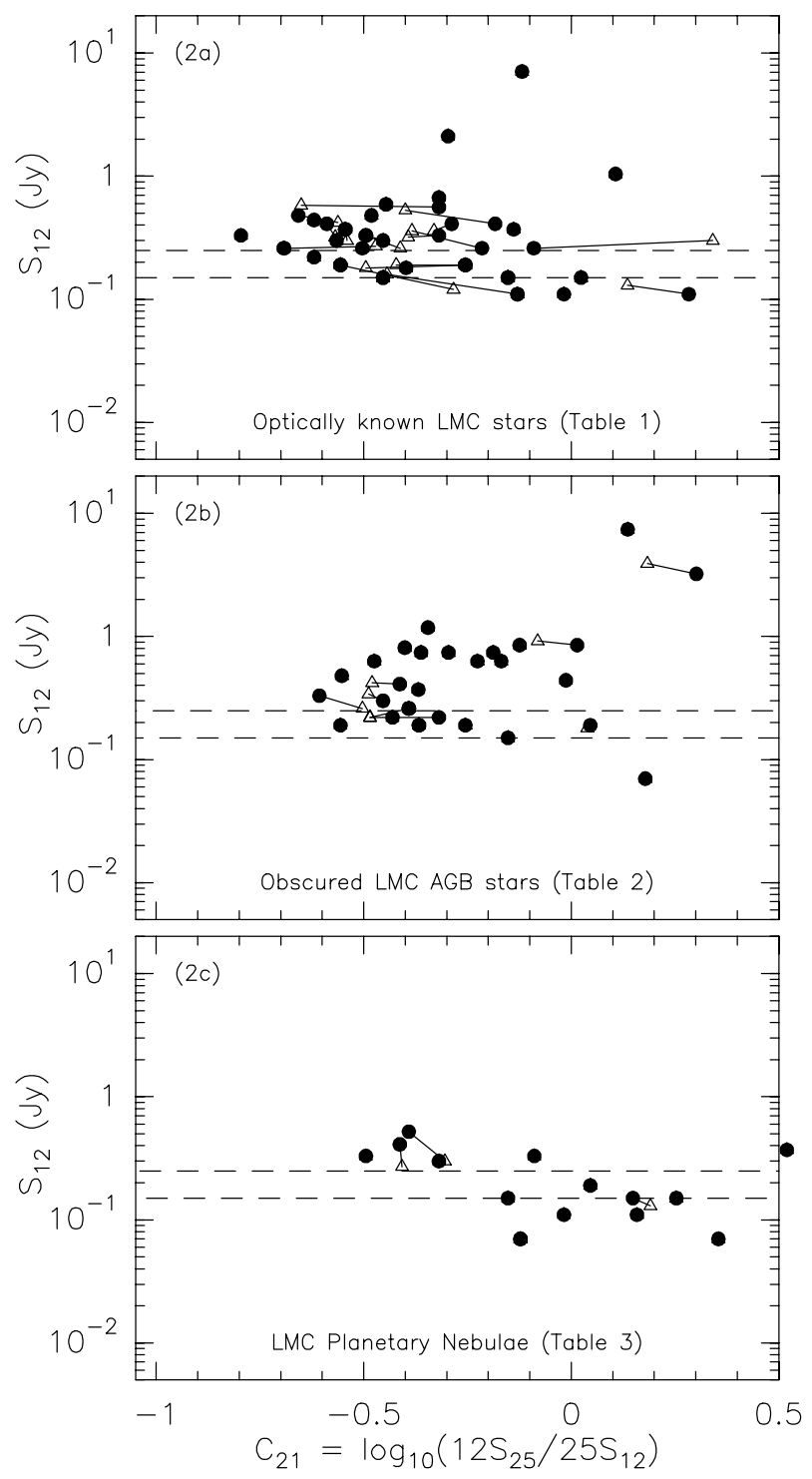

Fig. 2. Location of sources found in Table 1 (Fig. 2a), Table 2 (Fig. 2b), and Table 3 (Fig. 2c) in the $\left[C_{21}, S_{12}\right]$ diagram. Full dots correspond to the IRAS fluxes determined by Schwering \& Israel, open triangles correspond to IRAS fluxes determined by Reid et al. One can see that the disagreement between both determinations is often much larger than the "standard" adopted calibration uncertainty of $15 \%$ on IRAS fluxes (corresponding to \pm 0.13 on $C_{21}$ ). On average, taking into account the large uncertainty on IRAS fluxes (see Sect. 5), obscured AGB stars are colder than sources with optical counterparts, as expected

distribution, Rowan-Robinson \& Harris (1983) had to use a dust temperature at the inner radius of the dust shell of only typically $500 \mathrm{~K}$, far below the dust condensation temperature. This would reflect either a peculiar massloss history, or the fact that the dust condensates much further from the star than in $M$ giants. It might be that the same phenomenon occurs in some M supergiants of the LMC.
However, here we work with fluxes close to the detection limit and we should first invoke the uncertainty of these fluxes. Both Schwering \& Israel and Reid et al. give a typical calibration uncertainty of $15 \%$, leading to an uncertainty of \pm 0.13 on $C_{21}$, though Reid et al. note that this uncertainty can be much larger for some sources. In Table 8, for sources in common to Schwering \& Israel and Reid et al., we present a comparison of the various determinations of IRAS fluxes. Clearly, the disagreement between Schwering \& Israel and Reid et al. is often much larger than $15 \%$. In fact, as noted by Reid et al., there is a systematic disagreement for $S_{12}$ and $S_{25}$. Reid et al. underestimate $S_{12}$ and $S_{25}$ by typically $25 \%$ compared to Schwering \& Israel, and by $10 \%$ compared to the PSC. Reid et al. say that $10 \%$ is acceptable as it is inside the admitted $15 \%$ uncertainty. This is correct when one considers one source, but in a statistical sample they should not find any systematic deviations. Clearly, IRAS fluxes have not been determined with the same method by Schwering \& Israel and by Reid et al. As a consequence, there is also a systematic deviation in the value of $C_{21}$ which is generally underestimated by Reid et al. compared to Schwering \& Israel. The typical uncertainty on $C_{21}$ that we derive from Table 8 is \pm 0.25 , and hence only a few sources in Table 1 really have a cold $C_{21}$ colour (Fig. 2a). For them, one could also doubt that the IRAS source is actually associated with the optically identified star.

With such uncertainties in IRAS fluxes, the list of obscured AGB star candidates given in Table 4 probably misses a few objects whose 60 and/or $100 \mu \mathrm{m}$ fluxes are contaminated. Conversely, a few sources in Table 4 might be associated with HII regions. However, we think that this list is the most complete one can make in a systematic way, and is quite reliable as it can be seen in Table 1 and 2 for identified objects. This list contains 6 times more sources than known obscured AGB stars listed in Table 2. Therefore further studies should be performed to clearly identify them. We expect that many of them will be identified through the $I J K^{\prime}$ observations of the DEep Near Infrared Survey of the southern sky (DENIS).

Acknowledgements. We are very grateful to P. Whitelock and B.E Westerlund for their helpful comments. This research has made use of the Simbad database, operated at CDS, Strasbourg, France.

\section{References}

Blanco V.M., McCarthy M.F., Blanco B.M., 1980, ApJ 242, 938 (BMB)

Blanco V.M., McCarthy M.F., 1983, AJ 88, 1442

Blanco V.M., McCarthy M.F., 1990, AJ 100, 674 (BM)

Catchpole R.M., Feast M.W., 1981, MNRAS 197, 385

Elias J.H., Frogel J.A., Humphreys R.M., 1980, ApJ 242, L13

Elias J.H., Frogel J.A., Humphreys R.M., 1985, ApJS 57, 91

Elias J.H., Frogel J.A., Schwering P.B.W., 1986, ApJ 302, 675

Feast M.W., Catchpole R.M., Carter B.S., Roberts G., 1980, MNRAS 193, 377 
Frogel J.A., Blanco V.M., 1990, ApJ 365, 168 (BMB)

Frogel J.A., Richer H.B., 1983, ApJ 275, 84

Fouqué P., Le Bertre T., Epchtein N., Guglielmo F. Kerschbaum F., 1992, A\&AS 93, 151

Glass I.S., 1979, MNRAS 186, 317

Glass I.S., Reid N., 1985, MNRAS 214, 405

de Grijp M.H.K, Miley G.K., Lub J., 1987, A\&AS 70, 95

Groenewegen M.A.T., de Jong T., 1993, A\&A 267, 410

Henize K.G., 1956, ApJS 2, 315 (LHA)

Hughes S.M.G., 1989, AJ 97, 1634 (SHV)

Hughes S.M.G., Wood P.R., 1990, AJ 99, 784 (SHV)

Humphreys R.M., 1979, ApJS 39, 389

Israel F.P, Koorneef J., 1991, A\&A 248, 404

Jura M., Kleinmann S.G, 1990, ApJS 73, 769

Le Bertre T., 1992, A\&AS 94, 377

Loup C., Forveille T., Omont A., Paul J.F., 1993, A\&AS 99, 291

Lundgren K., 1988, A\&A 200, 85

Meatheringham S.J., Dopita M.A., 1991a, ApJS 75, 407

Meatheringham S.J., Dopita M.A., 1991b, ApJS 76, 1085

Morgan D.H., Good A.R., 1992, A\&AS 92, 571 (MG)

Neugebauer G., et al., 1984, ApJ 278, L1

Pottasch S.R., Bignell C., Olling R., Zijlstra A.A., 1988, A\&A 205, 248

Price S.D., Walker R.G., 1976, AFGL For Color Infrared Sky Survey, Environmental Research Papers, No. 576 (AFGL)

Rebeirot E., Azzopardi M., Westerlund B.E., 1993, A\&AS 97, 603 (RAW)

Rebeirot E., Martin N., Mianes P., Prévot L., Robin A., Rousseau J., Peyrin Y., 1983, A\&AS 51, 277 (RMMP)

Reid N., 1991, ApJ 382, 143

Reid N., Glass I.S., Catchpole R.M., 1988, MNRAS 232, 53 (GRV)
Reid N., Hughes S.M.G, Glass I.S., 1995, MNRAS 275, 331 (RHV)

Reid N., Tinney C., Mould J., 1990, ApJ 348, 98 (TRM)

Roche P.F., Aitken D.K., Smith C.H., 1993, MNRAS 262, 301

Sanduleak N., 1989, AJ 98, 825

Sanduleak N., MacConnell D.J., Philip A.G., 1978, PASP 90, 621 (SMP)

Sanduleak N., Philip A.G., 1977, Pub. Warner and Swasey Obs. 2, No. 5 (SP)

Schwering P.B.W., 1989, A\&AS 79, 105 (LI-LMC, LI-SMC)

Schwering P.B.W., Israel F.P., 1990, "Atlas and catalogue of infrared sources in the Magellanic Clouds". Kluwer Academic Publishers (LI-LMC, LI-SMC)

Stencel R.E., Pesce J.E., Bauer, W.H., 1989, AJ 97, 1120

Vassiliadis E., Dopita M.A., Morgan D.H., Bell F.J., 1992a, ApJS 83, 87

Vassiliadis E., Meatheringham S.J., Dopita M.A., 1992b, ApJ 394,489

Westerlund B.E., 1961, Uppsala Ann. Band 5 No. 1

Westerlund B.E., Olander N., Hedin B., 1981, A\&AS 43, 267 (WOH)

Westerlund B.E., Olander N., Richer A.B., Crabtree D.R., 1978, A\&AS 31, 61 (WORC)

Whitelock P.A., Feast M.W., Menzies J.W., Catchpole R.M., 1989, MNRAS 238, 769

Wood P.R., Bessell M.S., Fox M.W., 1983, ApJ 272, 99

Wood P.R., Bessell M.S., Whiteoack J.B., 1986, ApJ 306, L81

Wood P.R., Whiteoack J.B., Hughes S.M.G., Bessell M.S., Gardner F.F., Hyland A.R., 1992, ApJ 397, 552

Zijlstra A.A., Loup C., Waters L.B.F.M., Whitelock P.A., van Loon J.T., Guglielmo F., 1996, MNRAS 279, 32 (Paper II)

Zijlstra A.A., van Hoof P.A.M., Chapman J.M., Loup C., 1994, A\&A 290, 228 
Table 1. Optically known $\mathrm{M}$ and $\mathrm{C}$ stars

\begin{tabular}{|c|c|c|c|c|c|c|c|c|c|c|c|}
\hline LI & TRM & $\operatorname{RA}(1950)$ & $\operatorname{DEC}(1950)$ & $S_{12}$ & $S_{25}$ & $S_{60}$ & $S_{100}$ & $\mathrm{C}_{21}$ & $\mathrm{C}_{32}$ & group & identification \\
\hline$*_{5}$ & $\ldots$ & 003504.2 & .743617 & 0.30 & 0.22 & - & - & -0.5 & $<-0.1$ & 1 & JHKL, Ce(+?), nvar, V=14.6[3,7] \\
\hline$*_{44}$ & $\ldots$ & 004615.6 & -733956 & $0.11:$ & $0.11:$ & 4.1 & $\mathrm{C}$ & -0.3 & +1.2 & 1 & SkKM $25(\mathrm{~K} / \mathrm{M} ?, \mathrm{~V}=13.5)$ \\
\hline$*_{57}$ & $\ldots$ & 004742.8 & -734304 & 0.19 & 1.11 & 14.0 & 27.0 & +0.5 & +0.7 & 3 & SkKM $47 ? ? ?(\mathrm{~K} / \mathrm{M} ?)[5]$ \\
\hline 59 & $\ldots$ & 004757 & -7319 & $0.19:$ & 0.11 : & 2.5 & $\mathrm{C}$ & -0.6 & +1.0 & 1 & $\mathrm{SkKM} 50(\mathrm{~K} / \mathrm{M} ?, \mathrm{~V}=13.5)$ \\
\hline 139 & $\ldots$ & 005906 & -7307 & 0.19 & 0.11 : & 0.4 & $\mathrm{C}$ & -0.6 & +0.2 & 1 & HV $11417(\mathrm{M} 5 \mathrm{e})$ \\
\hline 140 & $\ldots$ & 005910 & .7155 & 0.19 & - & $\mathrm{C}$ & $\mathrm{C}$ & $<-0.3$ & - & 2 & HV 11423 (MOIab, $B=12.5)$ \\
\hline${ }^{*} 185$ & $\ldots$ & 010727.8 & -714006 & 0.41 & 0.44 & - & - & -0.3 & $<-0.4$ & 1 & HV $12956(\mathrm{M} 5 e)[3]$ \\
\hline 143 & $\ldots$ & 045358 & -6903 & 0.11 & 0.17 : & - & - & -0.1 & $<+0.0$ & 1 & WOH SG061 (M0, I=13.9, P=857) [i,w] \\
\hline${ }^{*} 153$ & $\ldots$ & 045424.6 & .684902 & 0.30 & 0.17 : & - & - & -0.6 & $<+0.0$ & 1 & SP $30-6(M 8, V=13.4, P=728)[i, L, w]$ \\
\hline${ }^{*} 181$ & $\ldots$ & 045518.4 & .682515 & 7.07 & 11.21 & 4.1 & 10.4: & -0.1 & -0.8 & 3 & WOH G064 (M7.5, I=12.4, P=930, OH) $[i, 1,2,5,6]$ \\
\hline${ }^{*} 183$ & $\ldots$ & 045520.5 & .693353 & 0.67 & 0.67 & 2.1: & $\mathrm{C}$ & -0.3 & +0.1 & 1 & WOH SG071 (M2, R=12.2) [i,7] \\
\hline${ }^{*} 253$ & $\ldots$ & 045808.7 & -701327 & 0.59 & 0.44 & - & - & -0.4 & $<-0.4$ & 1 & HV $2255(M 4, V=13.2, P=830)[d, g, i, j, 1, L, 7]$ \\
\hline${ }^{*} 383$ & 048 & 050415.9 & -672027 & 0.56 & 0.56 & . & - & -0.3 & $<-0.5$ & 1 & HV $888(\mathrm{M} 3 / 4 \mathrm{Ia}, \mathrm{V}=12.3, \mathrm{P}=850)[\mathrm{d}, \mathrm{e}, \mathrm{i}, \mathrm{l}, \mathrm{L}, \mathrm{o}, \mathrm{q}, \mathrm{v}]$ \\
\hline${ }^{*} 425$ & $\ldots$ & 050553.8 & -684304 & 0.26 & 0.17 ; & - & - & -0.5 & $<+0.0$ & 1 & HV $11977(M 1+, V=13.8)[i, L]$ \\
\hline 482 & $\ldots$ & 050812 & -6829 & 0.15 & 0.11 & 3.7 & 4.2 & -0.4 & +1.1 & 1 & WORC $90(\mathrm{C} ?, \mathrm{I}=12.8)[\mathrm{D}]$ \\
\hline$*_{517}$ & $\ldots$ & 050931.8 & -652535 & $0.15:$ & $0.22:$ & - & - & -0.2 & $<-0.1$ & 1 & $\mathrm{HV} 12185(\mathrm{M} 1, \mathrm{~V}=12.7)[\mathrm{i}, \mathrm{L}]$ \\
\hline${ }^{*} 612$ & 043 & 051249.6 & -672308 & 0.26 & 0.33 & - & - & -0.2 & $<-0.3$ & 1 & HV $2360(\mathrm{M} 2 / 4 \mathrm{Ia}, \mathrm{V}=1 \mathbf{3 . 8}, \mathrm{P}=409)[\mathrm{d}, e, \mathrm{i}, \mathrm{l}, \mathrm{L}, \mathrm{O}, \mathrm{q}, \mathrm{r}, \mathrm{v}]$ \\
\hline${ }^{*} 663$ & 036 & 051453.4 & -673036 & 0.26 & 0.44 & $0.8:$ & $\mathrm{C}$ & -0.1 & -0.1 & 1 & HV 916 (M3Ia, V=12.6, P=743) [d,e,i,l,L,q, v] \\
\hline${ }^{*} 728$ & $\ldots$ & 051703.4 & -692709 & 0.48 & 0.33 : & 8.3: & $\mathrm{C}$ & -0.5 & +1.0 & 1 & WOH G255 (M, I=12.1) [i] \\
\hline 833 & $\ldots$ & 052042 & -6636 & 0.22 & & - & - & $<-0.3$ & - & 1 & WOH G278 (M3S, V=15.6) [i,r] \\
\hline 869 & $\ldots$ & 052150 & .6933 & $0.15:$ & $0.11:$ & . & - & -0.5 & $<+0.2$ & 1 & $\mathrm{HV} 12017(\mathrm{M} 1, \mathrm{~V}=12.6)[\mathrm{i}, \mathrm{L}]$ \\
\hline${ }^{*} 932$ & 108 & 052335.7 & -654435 & 0.41 & 0.22 & $0.8:$ & 8.3: & -0.6 & +0.2 & 3,1 & HV $12793(\mathrm{M} 3 / 4, \mathrm{I}=11.2)[\mathrm{i}, \mathrm{v}, 4,7]$ \\
\hline${ }^{*} 976$ & $\ldots$ & 052445.0 & -694130 & 1.04 & 2.77 & $\mathrm{C}$ & $\mathrm{C}$ & +0.1 & - & 2 & WOH SG264 (M2, V=13.3) [i,L,5] \\
\hline$*_{1033}$ & 129 & 052608.4 & -661434 & 0.56 & 0.78 : & 8.3: & $\mathrm{C}$ & -0.2 & - & 3,1 & WOH SG281 $(M 3, V=11)[i, r, v]$ \\
\hline${ }^{*} 1038$ & $\ldots$ & 052611.5 & -660927 & $0.37:$ & 0.56 : & $\mathrm{C}$ & $\mathrm{C}$ & -0.1 & - & 2 & WOH SG282(M2, V=14.2) [i,L] \\
\hline${ }^{*} 1059$ & $\ldots$ & 052642.8 & .691317 & 0.33 & 0.22 : & - & - & -0.5 & $<-0.1$ & 1 & HV $2532(\mathrm{M} 4, \mathrm{~V}=13.4, \mathrm{P}=650)[\mathrm{i}, 1, \mathrm{~L}, \mathrm{o}]$ \\
\hline$\ldots .$. & 052 & 052733.5 & -671728 & 0.15 & & - & - & $<-0.2$ & - & 2 & HV $5845(\mathrm{M} 1 \mathrm{Ia}, \mathrm{V}=12.7)[\mathrm{d}, \mathrm{i}, \mathbf{L}, \mathrm{v}]$ \\
\hline$\ldots$. & 073 & 052736.5 & -665604 & 0.18 & 0.15 & - & - & -0.4 & $<+0.1$ & 1 & HV $963(\mathrm{M} 2 / 3 \mathrm{I}, \mathrm{V}=12.6)[\mathrm{i}, \mathrm{l}, \mathrm{L}, \mathrm{q}, \mathrm{v}, 7]$ \\
\hline$*_{1103}$ & & 052807.4 & -691545 & 0.30 & 0.22 & - & - & -0.5 & $<-0.1$ & 1 & SP $47-14(\mathrm{M} 1, \mathrm{~V}=12.9)[\mathrm{i}, \mathrm{L}, \mathrm{7}]$ \\
\hline 1107 & 065 & 052815 & -6702 & 0.19 & $0.11:$ & $0.4:$ & 2.1: & -0.6 & - & 3,2 & HV $5854(\mathrm{M} 3 / 4.5 \mathrm{I}, \mathrm{V}=12.7, \mathrm{P}=524)[\mathrm{g}, \mathrm{i}, \mathrm{l}, \mathrm{L}, \mathrm{n}, \mathrm{o}, \mathrm{q}, \mathrm{v}]$ \\
\hline 1121 & $\ldots$ & 052840 & -7014 & $0.19:$ & 0.11 & 4.1 & $6.2:$ & -0.6 & +1.2 & 1 & WOH G351 (M, I=12.4) [i] \\
\hline${ }^{*} 1125$ & $\ldots$ & 052840.6 & .680932 & 0.19 & 0.22 & . & - & -0.3 & $<-0.1$ & 1 & HV $2561($ MOIa, $\mathrm{V}=12.5)[\mathrm{d}, \mathrm{i}, \mathrm{L}, \mathrm{o}]$ \\
\hline 1145 & & 052920 & -6909 & 0.15 & $0.22:$ & $\mathrm{C}$ & $\mathrm{C}$ & -0.2 & - & 2 & HV $5870(\mathrm{M} 5, \mathrm{~V}=14.3, \mathrm{P}=627)[\mathrm{d}, \mathrm{g}, \mathrm{i}, \mathrm{j}, \mathrm{l}, \mathrm{L}, \mathrm{o}]$ \\
\hline 1155 & 069 & 052930 & -6658 & 0.19 & 0.22 & - & - & -0.3 & $<-0.1$ & 1 & HV $2586(\mathrm{M} 1 / 3.5 \mathrm{I}, \mathrm{V}=13.1, \mathrm{P}=487)[\mathrm{g}, \mathrm{i}, \mathrm{l}, \mathrm{L}, \mathrm{O}, \mathrm{v}]$ \\
\hline${ }^{*} 1163$ & $\ldots$ & 052950.4 & -691125 & 0.44 & 0.22 & 0.8 : & - & -0.6 & - & 1 & SP 46-39 (M1Ia, V $=10.6)[a, d, e, i, L, o]$ \\
\hline${ }^{*} 1170$ & 049 & 052959.9 & -672044 & 0.19 & 0.22 & & - & -0.3 & $<-0.1$ & 1 & SP 45-34 (M1Ia +B?, V=12.1) [a,d,e,i,L, $, 7,7]$ \\
\hline$*_{1172}$ & $\ldots$ & 053001.4 & -685932 & 0.26 & 0.11 & - & - & -0.7 & $<+0.2$ & 1 & $\mathrm{SP} 46-44(\mathrm{M} 1 \mathrm{Ia}, \mathrm{V}=11.7)[\mathrm{a}, \mathrm{d}, \mathrm{e}, \mathrm{i}, \mathrm{L}, \mathrm{o}, \mathrm{r}, 7]$ \\
\hline *1190 & 046 & 053025.8 & -672223 & 0.48 & 0.22 & 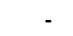 & . & -0.7 & $<-0.1$ & 1 & HV $2595(\mathrm{M} 1 \mathrm{la}, \mathrm{V}=12.4)[\mathrm{a}, \mathrm{d}, \mathrm{e}, \mathrm{i}, \mathrm{L}, \mathrm{o}, 7]$ \\
\hline *1212 & ... & 053106.4 & -691802 & 0.22 & $0.11:$ & $\mathrm{C}$ & $\mathrm{C}$ & -0.6 & - & 1 & HV $2604(\mathrm{M} 1, \mathrm{~V}=13.0, \mathrm{P}=664)[\mathrm{g}, \mathrm{i}, 1, \mathrm{~L}, \mathrm{o}]$ \\
\hline${ }^{*} 1223$ & $\ldots$ & 053123.5 & .692051 & 0.37 & 0.22 & 2.1 & 6.2 & -0.5 & +0.6 & 3 & HV $12998(\mathrm{M} 1, \mathrm{I}=10.5)[\mathrm{i}, \mathrm{L}, 7]$ \\
\hline${ }^{*} 1234$ & 089 & 053135.4 & -663152 & 0.33 & 0.22 : & - & - & -0.5 & $<-0.1$ & 1 & HV $990(\mathrm{M} 2 \mathrm{I}, \mathrm{V}=12.6)[\mathrm{i}, \mathrm{L}, \mathrm{v}, 7]$ \\
\hline${ }^{*} 1238$ & 101 & 053141.5 & -660453 & 0.41 & 0.44 & - & - & -0.3 & $<-0.4$ & 1 & WOH SG374 (M6, V=14.8) $[\mathrm{i}, \mathrm{L}, \nabla, 4,7]$ \\
\hline 1241 & 087 & 053151 & -6643 & $0.11:$ & 0.17 : & - & - & -0.1 & $<+0.0$ & 1 & SP $52-1(\mathrm{M} 3 / 4 \mathrm{I}, \mathrm{V}=12.9)[\mathrm{i}, \mathrm{L}, \mathrm{v}, 7]$ \\
\hline${ }^{*} 1281$ & 005 & 053245.1 & -675708 & 0.41 & 0.56 & - & . & -0.2 & $<-0.5$ & 1 & HV $996(\mathrm{M} 4 / 5 \mathrm{I}, \mathrm{V}=13.7, \mathrm{P}=760)[\mathrm{d}, \mathrm{i}, \mathrm{l}, \mathrm{L}, \mathrm{q}, \mathrm{v}]$ \\
\hline${ }^{*} 1294$ & 078 & 053308.3 & -665005 & 0.26 & $0.11:$ & - & - & -0.7 & $<+0.2$ & 1 & HV $12437(M 0.5 / 2, V=14.0, P=403)[i, 1, L, q, v]$ \\
\hline${ }^{*} 1304$ & 063 & 053329.8 & -670617 & 0.37 & 0.22 & - & - & -0.5 & $<-0.1$ & 1 & HV $5933(\mathrm{M} 4 \mathrm{I}, \mathrm{V}=11.9)[\mathrm{d}, \mathrm{i}, \mathrm{L}, \mathrm{o}, \nabla, 7]$ \\
\hline 1306 & $\ldots$ & 053330 & -6909 & 0.19 & - & - & - & $<-0.3$ & - & 2 & RMMP $600(M ?, V=13.9)[\mathrm{L}]$ \\
\hline 1360 & 062 & 053520 & -6705 & 0.19 & $0.11:$ & - & - & -0.6 & $<+0.2$ & 1 & HV $2700(\mathrm{M} 1 / 2 \mathrm{lab}, \mathrm{V}=13.3)[\mathrm{d}, \mathrm{i}, \mathrm{L}, \mathrm{q}, \mathrm{v}, 7]$ \\
\hline 1364 & & 053525 & .6746 & 0.11 & 0.22 & . & 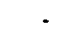 & +0.0 & $<-0.1$ & 1 & HV $1001(\mathrm{M} 4 / 5.5, \mathrm{~V}=14.7, \mathrm{P}=590)[\mathrm{d}, \mathrm{e}, \mathrm{i}, \mathrm{l}, \mathrm{Lq}]$ \\
\hline$* 1366$ & 068 & 053530.0 & -665753 & 0.33 & 0.22 & - & . & -0.5 & $<-0.1$ & 1 & WOH SG425(M3I, V=11.8) [i,v,7] \\
\hline${ }^{*} 1399$ & 067 & 053627.8 & -665725 & 0.33 & 0.33 & - & - & -0.3 & $<-0.3$ & 1 & HV $1004(\mathrm{M} 3 / 5.5 \mathrm{I}, \mathrm{V}=13.1, \mathrm{P}=555)[\mathrm{e}, \mathrm{i}, \mathrm{l}, \mathrm{L}, \mathrm{q}, \mathrm{v}]$ \\
\hline 1458 & $\ldots$ & 053830 & -6918 & $0.37:$ & $\mathrm{C}$ & $\mathrm{C}$ & $\mathrm{C}$ & - & - & 2 & RMMP $716(\mathrm{M}, \mathrm{V}=14.1)[\mathrm{L}]$ \\
\hline$*_{1470}$ & $\ldots$ & 053857.4 & -692208 & 2.11 & 2.22 & $\mathrm{C}$ & $\mathrm{C}$ & -0.3 & - & 2 & WOH SG453 (M0.5, V=13.3) [i,5] \\
\hline${ }^{*} 1543$ & $\ldots$ & 054110.4 & .692341 & 0.26 & - & - & - & $<-0.4$ & - & 1 & RMMP $749(\mathrm{M} ?, \mathrm{~V}=13.8)[\mathrm{L}]$ \\
\hline${ }^{*} 1553$ & $\ldots$ & 054122.9 & -691958 & 0.37 & $0.22:$ & $\mathrm{C}$ & $\mathrm{C}$ & -0.5 & - & 1 & HV $1017(\mathrm{M} 2 \mathrm{I}, \mathrm{V}=13.2)[\mathrm{d}, \mathrm{i}, \mathrm{L}]$ \\
\hline$*_{1559}$ & $\ldots$ & 054134.3 & -690013 & $0.15:$ & 0.33 & $\mathrm{C}$ & $\mathrm{C}$ & +0.0 & - & 2 & HV $5999(\mathrm{M} 1, \mathrm{~V}=13.4)[\mathrm{i}, \mathrm{L}]$ \\
\hline$*^{*} 1602$ & 135 & 054318.6 & .672856 & 0.15 & $0.22:$ & $0.8:$ & $\mathrm{C}$ & -0.1 & +0.2 & 3 & $(V=13.8)[v, 7]$ \\
\hline *1799 & $\ldots$ & 055707.3 & -682744 & 0.33 & $0.11:$ & - & - & -0.8 & +0.2 & 1 & WOH G639 (M, I=11.5) [i] \\
\hline${ }^{*} 1823$ & $\ldots$ & 060655.7 & -723826 & $0.26:$ & - & - & - & $<-0.4$ & - & 1 & WOH SG532(M4, I=12.1)[i] \\
\hline
\end{tabular}

Notes to Tables 1 and 2 : WOH G352 is also very close to LI-LMC 1125. LI-SMC 61 (PSC00483-7347) was not selected because it is mentioned as slightly extended in Schwering \& Israel; note that Whitelock et al. (1989) mention that this object could also be a pre-main-sequence star. LI-LMC 1341 is near a nebulosity.

Reference codes [] : a, Westerlund, 1961. D, Westerlund et al., 1978. d, Humphreys, 1979. e, Glass, 1979. g, Feast et al., 1980. i, Westerlund et al., 1981. j, Catchpole \& Feast, 1981. l, Wood et al., 1983. L, Rebeirot et al., 1983. n, Glass \& Reid, 1985. o, Elias et al., 1985. q, Reid et al., 1988. r, Lundgren, 1988. v, Reid et al., 1990. 1, Elias et al., 1986. 2, Wood et al., 1986. 3, Whitelock et al., 1989. 4, Reid, 1991. 5, Wood et al., 1992. 6, Roche et al., 1993. 7, Zijlstra et al., 1995. 
Source name codes : SkKM, Sanduleak, 1989. SP, Sanduleak \& Philip, 1977. WORC, Westerlund, Olander, Richer, \& Crabtree, 1978. WOH (SG for supergiants candidates in Table I, G for giants candidates in Table II), Westerlund, Olander, \& Hedin, 1981. RMMP, Rebeirot, Martin, Mianes, Prevot, et al., 1983. GRV, Reid, Glass, and Catchpole, 1988. SHV, Hughes, 1989; Hughes \& Wood, 1990

Additional names (Tab.1): LI-LMC 0143: SHV0453582-690242; LI-LMC 0153 : WOH SG066 = RMMP 045 = SHV0454257684856; LI-LMC 0253 : WOH SG097 = RMMP 087; LI-LMC 0383 : WOH SG140 = SP 29-33 = RMMP 151; LI-LMC 0425: WOH SG157 = RMMP 183; LI-LMC 0517: SP 35-1 = WOH SG179 = RMMP 215; LI-LMC 0612: SP 37-24 = WOH SG193 $=$ RMMP 239; LI-LMC 0663: SP 37-35 = WOH SG204 = RMMP 256; LI-LMC 0869: SP 47-6 = WOH SG241 = RMMP 308; LI-LMC 0932: WOH SG257; LI-LMC 0976: RMMP 339; LI-LMC 1038: RMMP 358; LI-LMC 1059: SP 46-16= WOH SG287 = RMMP 364: TRM 052: SP 45-16= WOH SG299 = RMMP 383; TRM 073: SP 45-18 = WOH SG301 = RMMP 384; LI-LMC 1103: WOH SG306 = RMMP 390; LI-LMC 1107: HV 5854 = SP 45-23 = WOH SG313 = RMMP 401; LI-LMC 1125: SP 46-32 = WOH SG319 = RMMP 408; LI-LMC 1145: SP 47-17 = WOH SG331 = RMMP 432; LI-LMC 1155: WOH SG337 = RMMP 444; LI-LMC 1163 : SP 46-39= WOH SG338 = RMMP 448; LI-LMC 1170 : WOH SG343= RMMP 468 = R 108; LI-LMC 1172 : WOH SG341 = RMMP 462; LI-LMC 1190: SP 45-38 = WOH SG349 = RMMP 482; LI-LMC 1212: SP 47-20= WOH SG358 = RMMP 505; LI-LMC 1223: SP 47-22 = WOH SG369 = RMMP 519; LI-LMC 1234: SP 51-6 = WOH SG371 = RMMP 531; LI-LMC 1238: RMMP 539; LI-LMC 1241: WOH SG375= RMMP 545; LI-LMC 1281: SP 46-59= WOH SG388= RMMP 575; LI-LMC 1294: WOH SG395 = RMMP 589 = GRV0533-6650; LI-LMC 1304: SP 52-18 = WOH SG401 = RMMP 606; LI-LMC 1360 : SP 52-29 = WOH SG422 = RMMP 656; LI-LMC 1364: WOH SG421 = RMMP 655; LI-LMC 1366: SP 52-32 ??; LI-LMC 1399: SP 52-35 = WOH SG432 = RMMP 683; LI-LMC $1553:$ SP 54-34 = WOH SG467 = RMMP 753; LI-LMC $1559:$ SP 54-40 = WOH SG473 = RMMP 761;

Table 2. Infrared AGB stars or SGs

\begin{tabular}{|c|c|c|c|c|c|c|c|c|c|c|c|}
\hline LI & TRM & $\mathrm{RA}(1950)$ & $\operatorname{DEC}(1950)$ & $\mathrm{S}_{12}$ & $S_{25}$ & $\mathrm{~S}_{60}$ & $\mathrm{~S}_{100}$ & $\mathrm{C}_{21}$ & $\mathrm{C}_{32}$ & group & identification \\
\hline *61 & $\ldots$ & 004822.1 & -734748 & 0.78 & 0.53 & $0.9:$ & $\mathrm{C}$ & -0.5 & -0.1 & 3 & JHKL [3] \\
\hline *119 & $\ldots$ & 005524.8 & -735129 & 0.44 & 0.22 & $\mathrm{C}$ & C & -0.6 & - & 1 & JHKL, $\mathrm{P}=800[3]$ \\
\hline *1825 & $\ldots$ & 042841.9 & .693715 & 0.22 & 0.17 & - & - & -0.4 & $<+0.0$ & 1 & JHKL [7] \\
\hline *1844 & $\ldots$ & 043727.3 & -683110 & 0.19 & 0.17 & - & - & -0.4 & $<+0.0$ & 1 & JHKL [7] \\
\hline$*_{4}$ & $\ldots$ & 044046.7 & .700054 & 0.81 & 0.67 & - & - & -0.4 & $<-0.6$ & 1 & JHKL [7] \\
\hline$*_{57}$ & $\ldots$ & 044938.4 & -695817 & 0.37 & 0.33 & - & - & -0.4 & $<-0.3$ & 1 & JHKL [7] \\
\hline$*_{60}$ & $\ldots$ & 044950.3 & -684253 & 1.18 & 1.11 & - & - & -0.3 & $<-0.8$ & 1 & JHKL [7] \\
\hline *77 & $\ldots$ & 045055.7 & -692232 & 0.74 & 1.00 & C & C & -0.2 & - & 2 & JHKL, $P=1290[5]$ \\
\hline$* 92$ & $\ldots$ & 045141.3 & -690249 & 0.63 & 0.78 & 1.2 & C & -0.2 & -0.2 & 1 & JHKL, $P=1090[5]$ \\
\hline${ }^{*} 121$ & $\ldots$ & 045300.4 & -691643 & 2.07 & 5.11 & 24.8 & 39.5 & +0.1 & +0.3 & $\mathbf{3}$ & JHKL, $P=1260:[5]$ \\
\hline${ }^{*} 141$ & $\ldots$ & 045354.2 & -682111 & 0.19 & 0.22 & - & - & -0.3 & $<-0.1$ & 1 & JHKL [7] \\
\hline *159 & $\ldots$ & 045432.0 & -700044 & 0.44 & 0.89 & 0.8 & 4.2: & -0.0 & -0.1 & 3 & JHKL, $P=1270$, OH [5] \\
\hline$* 198$ & $\ldots$ & 045542.1 & -675325 & 0.26 & 0.22 & - & - & -0.4 & $<-0.1$ & 1 & JHKL [7] \\
\hline$* 297$ & $\ldots$ & 050018.6 & -671221 & 0.44 & 0.44 & $0.8:$ & 2.1: & -0.3 & $<-0.1$ & 1 & JHKL [7] \\
\hline *310 & $\ldots$ & 050057.2 & -661658 & 0.26 & 0.22 & - & $\cdot$ & -0.4 & $<-0.1$ & 1 & JHKL [7] \\
\hline *528 & 023 & 050959.6 & -674019 & 0.19 & 0.44 & 0.8 & 4.2 & +0.0 & -0.1 & 3,1 & JHKL [7] \\
\hline$* 570$ & 004 & 051117.3 & -675549 & 0.41 & 0.33 & 1.2 & $2.1:$ & -0.4 & +0.2 & 1 & IJHKL $[4,7]$ \\
\hline${ }^{*} 571$ & 024 & 051118.4 & -673957 & 0.33 & 0.17 & - & • & -0.6 & $<+0.0$ & 1 & JHKL $[4,7]$ \\
\hline 578 & 072 & 051130 & -6656 & 0.19 & 0.11 : & $\mathrm{C}$ & C & -0.6 & - & 1,2 & IJHK $[4,7]$ \\
\hline${ }^{*} 1880$ & $\ldots$ & 051250.1 & -645503 & 0.15 & 0.22 & - & - & -0.2 & $<-0.1$ & 1 & JHKL [7] \\
\hline$* 793$ & 020 & 051903.5 & .674823 & 0.30 & 0.22 & - & - & -0.5 & $<-0.1$ & 1 & JHKL $[4,7]$ \\
\hline$\ldots$. & 088 & 052019.5 & -663853 & 0.16 & - & - & - & $<-0.2$ & - & 2 & IJHKL $[4,7]$ \\
\hline \multirow[t]{2}{*}{$* 861$} & 011 & 052137.4 & -675355 & 3.22 & 13.43 & 26.1 & 20.8: & +0.3 & -0.1 & 1 & JHKL [5] \\
\hline & 045 & 052821.3 & -672313 & 0.13 & - & - & - & $<-0.1$ & - & 2 & IJHK [4] \\
\hline *1137 & $\ldots$ & 052908.1 & -670003 & 0.07 & 0.22 & 1.2 & 4.2: & +0.2 & +0.4 & 3 & JHKL [7] \\
\hline *1153 & $\ldots$ & 052927.1 & -710444 & 0.74 & 0.78 & $\mathrm{C}$ & $\mathrm{C}$ & -0.3 & - & 2 & JHKL, $P=1040[5]$ \\
\hline *1157 & $\ldots$ & 052931.6 & -712141 & 0.19 & 0.17 & - & - & -0.4 & $<+0.0$ & 1 & JHKL [7] \\
\hline *1164 & $\ldots$ & 052952.2 & -695727 & 0.85 & 1.33 & $1.2:$ & $\mathrm{C}$ & -0.1 & -0.4 & 1 & JHKL, $P=1280$, OH $[5]$ \\
\hline *1177 & 079 & 053005.6 & -665115 & $0.26:$ & 0.22 & - & - & -0.4 & $<-0.1$ & 1 & JHKL $[4,7]$ \\
\hline *1286 & 060 & 053254.7 & -670854 & 0.85 & 1.83 & $0.4:$ & - & +0.0 & -1.0 & 1 & JHKL, $P=1260$, OH $[4,5,7]$ \\
\hline *1341 & $\ldots$ & 053441.0 & -694913 & 7.40 & 21.09 & 20.7 & 20.8 & +0.1 & -0.4 & 1 & $\operatorname{KLM}[1,2,6]$ \\
\hline *1345 & $\ldots$ & 053448.4 & -702448 & 0.48 & 0.28 & - & - & -0.6 & $<-0.2$ & 1 & $\mathrm{HK}[7]$ \\
\hline${ }^{*} 1382$ & 077 & 053600.8 & -664826 & 0.22 & 0.22 & $0.8:$ & - & -0.3 & +0.2 & 1 & $\mathrm{JHK}[4,7]$ \\
\hline *1506 & $\ldots$ & 054013.2 & -695646 & 0.63 & 0.89 & $\mathrm{C}$ & $\mathrm{C}$ & -0.2 & - & 2 & JHKL, $P=1390$, OH $[5]$ \\
\hline *1756 & $\ldots$ & 055036.1 & .705358 & 0.63 & 0.44 & - & - & -0.5 & $<-0.4$ & 1 & JHKL $[7]$ \\
\hline *1790 & $\ldots$ & 055550.7 & -700024 & 0.74 & 0.67 & - & - & -0.4 & $<-0.6$ & 1 & JHKL $[7]$ \\
\hline
\end{tabular}


Table 3. Planetary Nebulae

\begin{tabular}{|c|c|c|c|c|c|c|c|c|c|c|c|}
\hline LI & TRM & $\mathrm{RA}(1950)$ & $\operatorname{DEC}(1950)$ & $s_{12}$ & $\mathrm{~S}_{25}$ & $S_{60}$ & $S_{100}$ & $\mathrm{C}_{21}$ & $\mathrm{C}_{32}$ & group & identification \\
\hline$*_{229}$ & $\ldots$ & 002153.8 & .735400 & 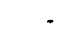 & 0.22 & & & $>-0.2$ & $<-0.1$ & 1 & SMP 2-1 \\
\hline$*_{12}$ & $\cdots$ & 003933.7 & -740345 & & 0.22 & $0.4:$ & C & $>-0.2$ & -0.1 & 1 & SMP 2-6 \\
\hline$*_{50}$ & $\cdots$ & 004647.2 & .731430 & 0.19 & 1.00 & 4.5 & 4.2 & +0.4 & +0.3 & 3 & SMP 2-11 \\
\hline 91 & $\ldots$ & 005124 & -7301 & - & 0.44 & $\mathrm{C}$ & $\mathrm{C}$ & +0.1 & - & & SMP 2-19 \\
\hline$*_{2}$ & $\ldots$ & 043849.4 & .704247 & $0.11:$ & 0.33 & 0.2 : & $1.0:$ & +0.2 & -0.6 & 3 & SMP 1-01 \\
\hline$* 72$ & $\ldots$ & 045030.0 & .693845 & 0.15 & 0.56 & 2.1: & C & +0.2 & +0.2 & 3 & SMP $1-08$ \\
\hline$* 89$ & $\ldots$ & 045135.4 & -671014 & 0.33 & 0.56 & $\mathrm{C}$ & C & -0.1 & - & 2 & SMP 1-11 \\
\hline 303 & $\ldots$ & 050030 & -7032 & 0.22 & & & & -0.3 & - & 1 & SMP $1-13$ \\
\hline$*_{438}$ & $\ldots$ & 050639.0 & -690312 & 0.07 : & $0.11:$ & 1.7 & 4.2 & -0.1 & +0.8 & 3 & SMP $1-24$ \\
\hline$*_{481}$ & $\ldots$ & $\begin{array}{lll}05 & 08 & 11.9\end{array}$ & -685533 & & 0.44 & 2.1: & $\mathrm{C}$ & $>+0.1$ & +0.3 & 3 & SMP $1-28$ \\
\hline${ }^{*} 483$ & & $\begin{array}{llll}05 & 08 & 15.5\end{array}$ & -684421 & 0.07 : & 0.11 & 2.1: & C & -0.1 & +0.9 & 3 & SMP 1-29 \\
\hline 513 & 012 & 050925.7 & -675103 & 0.15 : & 0.44 & & 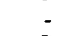 & +0.1 & $<-0.4$ & 1 & SMP 1-31 \\
\hline 557 & & 051052.9 & -6839 & 0.33 & 0.22 & 3.3: & $\mathrm{C}$ & -0.5 & +0.8 & 1 & SMP $1-36$ \\
\hline$*_{823}^{*}$ & $\ldots$ & 052016.4 & -665549 & 0.37 & 2.55 & 27.3 & 39.5 & +0.5 & +0.6 & 3 & MG 36 \\
\hline \multirow[t]{2}{*}{836} & & 052045 & -6958 & 0.07 : & 0.33 & & 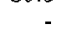 & +0.4 & $<-0.3$ & 1 & SMP $1-48$ \\
\hline & 143 & 052135.7 & .670248 & 0.08 & & & & +0.1 & - & 2 & SMP 1-53 \\
\hline 924 & . & 052320 & .7123 & 0.11 & $0.22:$ & 1.7 & 4.2 & -0.0 & +0.5 & 3 & SMP $1-55$ \\
\hline$* 982$ & ... & 052450.6 & -75 & 0.15 & 0.22 & & & -0.2 & -0.1 & 1 & SMP 1-58 \\
\hline$*_{1014}$ & & 052542.1 & .713545 & 0.19 : & 0.44 & $\mathrm{C}$ & $\mathrm{C}$ & +0.0 & & 2 & SMP 1-62 \\
\hline$*^{*} 1149$ & 054 & $\begin{array}{ll}05 & 29 \\
20.7\end{array}$ & -671544 & 0.41 & 0.33 & & - & -0.4 & $<-0.3$ & 1 & SMP 1-69 \\
\hline & 058 & 053240 & -6710 & 0.52 & 0.44 & - & - & -0.4 & $<-0.4$ & 1 & MG 58 \\
\hline$*_{1513}$ & 148 & 054028.1 & -661903 & & 0.44 & & - & +0.1 & $<-0.4$ & 1 & SMP $1-85$ \\
\hline 1707 & 140 & 054725 & -6928 & 0.30 & 0.30 & 8.3 & 29.1 & -0.3 & +1.1 & 3 & SMP 1-92 \\
\hline
\end{tabular}

Notes to Table 3 : "SMP" comes from Sanduleak, McConnell, and Philip (1978), and "MG" from Morgan \& Good (1992). We have systematically searched for IRAS counterparts of PNe listed in Meatheringham and Dopita (1991a, 1991b), Vassiliadis et al. (1992a, 1992b), and Morgan and Good (1992). Zijlstra et al. (1994) find in addition IRAS counterparts to the PNe SMP 1-06, SMP 1-61, SMP 1-98, MG 45 and MA 18; these sources are not listed in Table 3 because they are located in the outer parts of the LMC and are outside the area studied by Schwering \& Israel.

Table 4. Unidentified sources from group 1

\begin{tabular}{|c|c|c|c|c|c|c|c|c|}
\hline LI & TRM & $\mathrm{RA}(1950)$ & $\operatorname{DEC}(1950)$ & $\mathrm{S}_{12}$ & $S_{25}$ & $\mathrm{~S}_{60}$ & $C_{21}$ & $\mathrm{C}_{32}$ \\
\hline 223 & $\ldots$ & 001621 & .7328 & 0.22 & & & $<-0.3$ & \\
\hline 224 & $\ldots$ & 001621 & -7403 & & 0.33 & - & $>+0.0$ & $<-0.3$ \\
\hline$*_{233}$ & $\ldots$ & 002603.0 & $\begin{array}{llll}-73 & 15 & 18\end{array}$ & 0.22 & 2.20 & 6.6 & +0.7 & +0.1 \\
\hline 235 & $\cdots$ & 002637 & .7356 & & 0.22 & 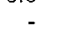 & $>-0.2$ & $<-0.1$ \\
\hline${ }^{*} 25$ & $\cdots$ & 004259.9 & .731358 & & 0.67 & 1.7 & $>0.3$ & +0.0 \\
\hline 34 & $\ldots$ & 004436 & .7408 & $0.33:$ & & 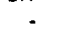 & $<-0.5$ & - \\
\hline 37 & $\ldots$ & 004451 & .7344 & 0.22 & 0.22 & 2.1 & -0.3 & +0.6 \\
\hline 40 & $\cdots$ & 004536 & -7257 & 0.30 & & & $<-0.5$ & - \\
\hline 48 & $\ldots$ & 004634 & -7301 & 0.19 & $0.11:$ & $\mathrm{C}$ & -0.6 & - \\
\hline$* 53$ & $\cdots$ & 004726.1 & -735007 & 0.11 : & 0.11 : & $0.4:$ & -0.3 & +0.2 \\
\hline 180 & $\ldots$ & 010545 & -7404 & 0.26 & - & - & $<-0.4$ & - \\
\hline 1 & $\ldots$ & 043836 & -7053 & 0.48 & - & . & $<-0.7$ & - \\
\hline 6 & $\cdots$ & 044136 & -7128 & 0.22 & - & - & $<-0.3$ & - \\
\hline${ }^{*} 31$ & $\ldots$ & 044722.0 & -682943 & 0.30 & $0.22:$ & & -0.5 & $<-0.1$ \\
\hline$*_{61}$ & $\ldots$ & 044952.2 & -712122 & $0.15:$ & $0.11:$ & 0.2 & -0.5 & -0.1 \\
\hline$*_{62}$ & $\cdots$ & 044953.0 & -692509 & 0.11 : & 0.78 & 2.1 & +0.5 & +0.0 \\
\hline$* 66$ & $\ldots$ & 045022.7 & -694532 & 0.26 & $0.22:$ & 0.8 & -0.4 & +0.2 \\
\hline 88 & $\ldots$ & 045130 & -6847 & 0.22 & $0.11:$ & - & -0.6 & $<+0.2$ \\
\hline 96 & $\ldots$ & 045150 & -6704 & 0.37 & 0.33 & $\mathrm{C}$ & -0.4 & - \\
\hline 97 & $\ldots$ & 045150 & -7030 & 0.26 & & . & $<-0.4$ & - \\
\hline$* 99$ & $\cdots$ & 045151.1 & .685223 & 0.37 & 0.22 & - & -0.5 & $<-0.1$ \\
\hline 100 & $\cdots$ & 045155 & -6715 & 0.26 & 0.11 : & $\mathrm{C}$ & -0.7 & \\
\hline 109 & $\ldots$ & 045220 & -6727 & 0.22 & $0.22:$ & - & -0.3 & $<-0.1$ \\
\hline 116 & $\ldots$ & 045245 & -6702 & 0.22 & 0.22 & 2.9 & -0.3 & +0.7 \\
\hline 129 & $\cdots$ & 045320 & -6809 & 0.33 & 0.22 & 0.8 & -0.5 & +0.2 \\
\hline *136 & $\ldots$ & 045335.3 & -661631 & 0.19 & 0.33 & $0.4:$ & -0.1 & -0.3 \\
\hline 155 & $\cdots$ & 045430 & -6640 & 0.22 & 0.22 & 1.2: & -0.3 & +0.4 \\
\hline$* 175$ & $\ldots$ & 045510.1 & .692841 & 0.33: & $0.22:$ & 1.2: & -0.5 & +0.4 \\
\hline$* 176$ & $\ldots$ & 045513.1 & -660558 & $0.15:$ & 0.33 & 0.8 & +0.0 & +0.0 \\
\hline 179 & $\ldots$ & 045515 & -6624 & 0.22 & 0.22 & 8.3 & -0.3 & +1.2 \\
\hline 197 & $\ldots$ & 045540 & -6837 & 0.33 & 0.11 & $\mathrm{C}$ & -0.8 & \\
\hline$* 203$ & $\ldots$ & 045557.3 & .693122 & 0.44 & 0.44 & 0.8 : & -0.3 & -0.1 \\
\hline 206 & $\ldots$ & 045620 & .6620 & 0.37 & 0.33 & 4.1 & -0.4 & +0.7 \\
\hline 207 & $\ldots$ & 045620 & -6938 & 0.19 & 0.17 & $\mathrm{C}$ & -0.4 & \\
\hline 212 & $\cdots$ & 045625 & -6911 & 0.15 & 0.11 & $0.8:$ & -0.4 & +0.5 \\
\hline$* 218$ & $\ldots$ & 045643.5 & -685719 & 0.19 & 0.11 & $\mathrm{C}$ & -0.6 & \\
\hline 220 & $\cdots$ & 045650 & -6650 & 0.19 & $0.11=$ & - & -0.6 & $<+0.2$ \\
\hline 237 & $\ldots$ & 045730 & -6913 & 0.30 & $0.11:$ & C & -0.8 & - \\
\hline
\end{tabular}

\begin{tabular}{|c|c|c|c|c|c|c|c|c|}
\hline LI & TRM & $\mathrm{RA}(1950)$ & $\operatorname{DEC}(1950)$ & $\mathrm{S}_{12}$ & $\mathrm{~S}_{25}$ & $S_{60}$ & $\mathrm{C}_{21}$ & $\mathrm{C}_{32}$ \\
\hline 256 & $\ldots$ & 045810 & -6909 & 0.15 & 0.11 & $0.8:$ & -0.4 & +0.5 \\
\hline 263 & $\ldots$ & 045830 & -6857 & 0.26 & 0.22 & 3.3 & -0.4 & +0.8 \\
\hline$*_{273}$ & $\ldots$ & 045848.1 & -681139 & 0.30 & 0.33 & & -0.3 & \\
\hline$*_{293}$ & $\ldots$ & 050002.0 & -692145 & 0.15 & 0.11 & C & -0.5 & \\
\hline$* 312$ & $\cdots$ & 050101.7 & -673920 & 0.22 & 0.17 : & 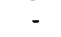 & -0.4 & $<+0.0$ \\
\hline 335 & $\ldots$ & 050212 & .7126 & $0.11:$ & 0.22 : & & +0.0 & $<-0.1$ \\
\hline$* 347$ & & 050245.2 & -690900 & 0.33 & 0.33 & 2.9: & -0.3 & +0.6 \\
\hline *372 & $\ldots$ & 050352.5 & -685715 & 0.37 & 0.33 & $\mathrm{C}$ & -0.4 & \\
\hline$* 382$ & & 050413.7 & -682945 & $0.15:$ & $0.11:$ & 1.7 & -0.4 & +0.8 \\
\hline *384 & & 050416.6 & -6827 & $0.15:$ & 0.33 & $0.8:$ & +0.0 & +0.0 \\
\hline$* 416$ & 134 & $05 \quad 0527.0$ & -6739 & & 0.33 & 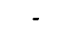 & +0.0 & $<-0$ \\
\hline${ }^{*} 436$ & $\ldots$ & 050620.6 & -690808 & $0.11:$ & 0.33 & - & +0.2 & $<-0.3$ \\
\hline 444 & & 050640 & -6941 & 0.22 & $0.17:$ & & -0.4 & $<+0.0$ \\
\hline$*_{463}$ & 009 & 050720.0 & -675243 & 0.22 & 0.44 & $0.8:$ & +0.0 & -0.1 \\
\hline & 133 & 0507 & -654227 & 0.17 & 0.22 & & -0.2 & $<-0.1$ \\
\hline$*_{478}$ & & 050 & -685 & 0.41 & 0.33 & 5.0 & & +0.8 \\
\hline$*_{484}$ & $\ldots$ & $0508 \quad 19.6$ & -70554 & 0.11 : & $0.11:$ & 0.8 & -0.3 & +0.5 \\
\hline 493 & $\ldots$ & 050840 & -6857 & $0.11:$ & $0.11:$ & C & -0.3 & \\
\hline 499 & $\ldots$ & 050850 & -70 & 15 & 0.11 & 1.2 & -0.4 & +o. \\
\hline 507 & $\cdots$ & 050910 & -6 & 26 & 0.22 & 1.7 : & -0.4 & +0 \\
\hline$*_{508}$ & $\ldots$ & 0509 & .690 & 0.2 & 0.22 & C & -0.4 & \\
\hline 512 & $\ldots$ & 05092 & -7010 & 0.1 & 0.17 & - & -0.4 & $<+0.0$ \\
\hline 530 & $\ldots$ & 051 & & 22 & 0.22 & 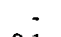 & -0.3 & $<-0.1$ \\
\hline$*_{535}$ & $\ldots$ & 051 & 58 & 11 & 0.11 : & 2.1: & & +0.9 \\
\hline 541 & & 0510 & .69 & 0.26 & 0.22 & 0.8 & -0.4 & +0.2 \\
\hline$*_{567}$ & 100 & 051105.5 & .661635 & 0.19 & 0.33 & & -0.1 & $<-0.3$ \\
\hline$*_{568}$ & $\ldots$ & 0511 & -68 & 0.22 & 0.22 & 5.0 & -0.3 & +1.0 \\
\hline 569 & $\ldots$ & 05111 & -69 & 0.26 & 0.17 & & & $<+0.0$ \\
\hline$*^{*} 575$ & $\ldots$ & 051120.1 & -693907 & 0.30 & 0.22 & $1.7:$ & -0.4 & +0.5 \\
\hline 576 & $\ldots$ & 051124 & .71 & 0.2 & & & -0.3 & \\
\hline$*^{*} 584$ & $\ldots$ & & 37 & 0. & 0.22 & 1.7: & -0.3 & +0.5 \\
\hline 595 & $\ldots$ & 05121 & -6753 & 0.15 & $0.11:$ & & & $<+0.2$ \\
\hline$*^{*} 603$ & $\ldots$ & 051232. & -703552 & 0.41 & 0.44 & $0.8:$ & -0.3 & -0.1 \\
\hline$*_{615}$ & $\ldots$ & 051 & -691927 & & & & -0.3 & \\
\hline 622 & $\ldots$ & & -7 & & 0.2 & 0.8 & -0.3 & +0.2 \\
\hline${ }^{*} 623$ & $\ldots$ & & & & & $\mathrm{C}$ & -0.4 & \\
\hline 633 & $\ldots$ & 05133 & -6944 & 0.22 & 0.22 & 1.2 & -0.3 & +0.4 \\
\hline 634 & $\ldots$ & 0513 & -69 & 0.4 & 0.44 & 2.1: & -0.4 & +0.3 \\
\hline 644 & $\ldots$ & 051400 & -6909 & 0.15 & 0.22 & & -0.2 & -0.1 \\
\hline
\end{tabular}


Table 4. continued

\begin{tabular}{|c|c|c|c|c|c|c|c|c|}
\hline $\mathbf{L I}$ & TRM & $\mathrm{RA}(1950)$ & $\operatorname{DEC}(1950)$ & $\mathrm{s}_{12}$ & $\mathrm{~S}_{25}$ & $\mathbf{S}_{60}$ & $\mathrm{C}_{21}$ & $\mathrm{C}_{32}$ \\
\hline & 156 & $0514 \quad 16.5$ & -662156 & & 0.31 & - & $>+0.0$ & $<-0.3$ \\
\hline${ }^{*} 671$ & $\ldots$ & $\begin{array}{lll}05 & 15 & 03.5\end{array}$ & -694236 & 0.30 & 0.44 & - & -0.2 & $<-0.4$ \\
\hline 686 & $\ldots$ & 051536 & .7104 & 0.26 & & . & $<-0.4$ & - \\
\hline$* 687$ & $\ldots$ & 051537.6 & .685224 & 0.11 & $0.11:$ & . & -0.3 & $<+0.2$ \\
\hline 694 & $\ldots$ & $05 \quad 1550$ & -6827 & 0.11 & 0.11 & - & -0.3 & $<+0.2$ \\
\hline 695 & $\ldots$ & 051550 & -6927 & 0.26 & 0.22 & C & -0.4 & - \\
\hline 696 & $\ldots$ & 051550 & -7031 & 0.19 & 0.11 : & . & -0.6 & $<+0.2$ \\
\hline 699 & $\ldots$ & 051600 & .6806 & 0.26 & 0.11 : & - & -0.7 & $<+0.2$ \\
\hline 700 & $\ldots$ & 051600 & -6811 & 0.11 & 0.11 : & - & -0.3 & $<+0.2$ \\
\hline 713 & $\ldots$ & 051630 & -6950 & 0.19 & $0.11:$ & C & -0.6 & - \\
\hline 721 & $\ldots$ & 051650 & -6803 & 0.22 & 0.22 & 1.7: & -0.3 & +0.5 \\
\hline 722 & $\ldots$ & 051650 & -6957 & 0.26 & & - & $<-0.4$ & 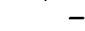 \\
\hline *726 & $\ldots$ & 051701.1 & .713711 & & 0.44 & - & $>+0.1$ & $<-0.4$ \\
\hline 730 & $\ldots$ & 051710 & -6822 & $0.19:$ & $0.22:$ & & -0.3 & $<-0.1$ \\
\hline \multirow[t]{2}{*}{$* 742$} & & 051738.5 & -692243 & 0.37 & 0.22 : & $\mathrm{C}$ & -0.5 & \\
\hline & 083 & 051741.0 & -664458 & 0.21 & 0.20 & - & -0.3 & $<-0.1$ \\
\hline 751 & 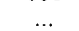 & 051750 & -6852 & 0.15 & 0.11 & $\mathrm{C}$ & -0.5 & \\
\hline 752 & $\ldots$ & 051750 & .7001 & 0.19 & 0.11 & 0.8 & -0.6 & +0.5 \\
\hline 767 & $\ldots$ & 051815 & .6948 & 0.15 & 0.11 & 0.8 & -0.4 & +0.5 \\
\hline 770 & $\ldots$ & 051820 & .6933 & 0.26 & 0.33 & & -0.2 & $<-0.3$ \\
\hline 799 & $\ldots$ & 051920 & .7022 & 0.15 & 0.11 : & 1.2 & -0.4 & +0.7 \\
\hline 802 & ... & 051930 & .6705 & 0.19 & $0.11:$ & 0.8 & -0.6 & +0.5 \\
\hline 805 & & 051930 & .6953 & 0.30 & 0.22 & $\mathrm{C}$ & -0.5 & - \\
\hline 812 & 006 & 051940 & -6757 & 0.15 & 0.11 & $\mathrm{C}$ & -0.5 & - \\
\hline$*_{825}$ & $\ldots$ & 052020 & .6913 & 0.33 & 0.33 & 4.1: & -0.3 & +0.7 \\
\hline 835 & $\ldots$ & 052045 & -6851 & 0.37 & 0.22 & $\mathrm{C}$ & -0.5 & - \\
\hline 849 & & 052120 & -7007 & 0.19 & $0.11:$ & $\mathrm{C}$ & -0.6 & - \\
\hline$* 855$ & 014 & 052129.1 & -674945 & 0.41 & 2.66 & 6.2 & +0.5 & +0.0 \\
\hline 868 & $\ldots$ & 052150 & -6841 & 0.22 & 0.22 & 1.7 & -0.3 & +0.5 \\
\hline 886 & $\ldots$ & 052220 & -7013 & $0.11:$ & $0.11:$ & $0.8:$ & -0.3 & +0.5 \\
\hline 890 & $\ldots$ & 052230 & .7009 & 0.11 : & $0.11:$ & $\mathrm{C}$ & -0.3 & \\
\hline 904 & & 052255 & -6721 & 0.19 & $0.11:$ & - & -0.6 & $<+0.2$ \\
\hline 914 & 059 & 052310 & -6710 & 0.37 & 0.33 & $\mathrm{C}$ & -0.4 & \\
\hline$* 918$ & $\ldots$ & $05 \quad 2313.8$ & -711125 & & 0.22 & 0.4 & -0.2 & -0.1 \\
\hline 935 & $\ldots$ & 052340 & -6958 & 0.30 & 0.22 & $0.8:$ & -0.4 & +0.2 \\
\hline$*_{937}$ & & 052342.8 & .700045 & $0.19:$ & 0.44 & $0.8:$ & +0.0 & -0.1 \\
\hline$* 938$ & 001 & 052343.8 & -675515 & $0.15:$ & 1.66 & 4.1: & +0.7 & +0.0 \\
\hline 939 & $\ldots$ & 052345 & -6850 & $0.15:$ & 0.11 : & 2.5 : & -0.4 & +1.0 \\
\hline 942 & $\ldots$ & 052350 & -6951 & 0.33 & $0.22:$ & $0.8:$ & -0.5 & +0.2 \\
\hline$* 948$ & & 052400.8 & -680948 & 0.11 : & 1.33 & 4.1 & +0.8 & +0.1 \\
\hline${ }^{*} 957$ & 016 & 052416.1 & -674818 & 0.19 & 0.22 & & -0.3 & $<-0.1$ \\
\hline 980 & & 052450 & -6852 & 0.30 : & & & $<-0.5$ & \\
\hline$* 986$ & $\ldots$ & 052456.6 & .695628 & 0.48 & 0.33 & 4.1: & -0.5 & +0.7 \\
\hline${ }^{*} 987$ & $\ldots$ & 052500 & -6918 & 0.44 & 0.22 & C & -0.6 & - \\
\hline 995 & $\ldots$ & 052512 & .7137 & 0.19 & 0.11 & $\mathrm{C}$ & -0.6 & 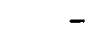 \\
\hline 997 & . & 052518 & .7153 & 0.19 & $0.11:$ & - & -0.6 & $<+0.2$ \\
\hline & $\ldots$ & 052530 & .7151 & 0.19 & 0.17 & - & -0.4 & $<+0.0$ \\
\hline \multirow[t]{2}{*}{1012} & & 052540 & -6823 & 0.26 & 0.22 & 2.1: & -0.4 & +0.6 \\
\hline & 158 & 052551.6 & .661313 & & 0.20 & & -0.2 & -0.1 \\
\hline 1024 & & 052600 & .6922 & 0.37 & 0.33 & 5.0 & -0.4 & +0.8 \\
\hline 1028 & $\ldots$ & 052600 & .7106 & 0.26 & 0.11 : & - & -0.7 & +0.2 \\
\hline$*^{*} 1029$ & $\ldots$ & 052602.2 & -671723 & $0.15:$ & $0.11:$ & $\mathrm{C}$ & -0.5 & \\
\hline$*^{*} 1031$ & $\cdots$ & 052605.1 & .701023 & & 0.17 & & -0.3 & $<+0.0$ \\
\hline 1055 & 040 & 052640 & .6726 & 0.19 & 0.11 : & $\mathrm{C}$ & -0.6 & \\
\hline 1061 & & 052645 & -6612 & 0.26 & 0.22 & $2.9:$ & -0.4 & +0.7 \\
\hline${ }^{*} 1082$ & 095 & 052718.1 & -662452 & 0.30 & 0.44 & 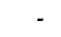 & -0.2 & $<-0.4$ \\
\hline${ }^{*} 1092$ & & 052748.2 & -694205 & 0.37 & 0.44 & & -0.2 & $<-0.4$ \\
\hline$*_{1101}$ & $\ldots$ & $\begin{array}{lll}05 & 28 & 03.8\end{array}$ & -703910 & 0.15 : & 0.11 & 1.7: & & +0.8 \\
\hline 1119 & $\ldots$ & 052840 & -7000 & 0.26 & 0.11 & & -0.7 & +0.2 \\
\hline 1123 & $\ldots$ & 052840 & .7057 & 0.11 & $0.11:$ & 0.8 : & -0.3 & +0.5 \\
\hline & & 052915 & .7007 & 0.26 & $0.11:$ & - & -0.7 & +0.2 \\
\hline \multirow[t]{2}{*}{1146} & & 052920 & -6945 & 0.26 & & & $<-0.4$ & \\
\hline & 103 & 052952.2 & -655223 & 0.14 & 0.23 & - & -0.1 & $<-0.1$ \\
\hline${ }^{*} 1179$ & & 053012.2 & .705653 & 0.22 : & $0.22:$ & 2.1 & -0.3 & +0.6 \\
\hline$*^{*} 1186$ & 075 & 053020.1 & -665504 & 0.22 & 0.22 & & -0.3 & $<-0.1$ \\
\hline 1196 & $\ldots$ & 053035 & -6942 & & 0.22 & - & $>-0.2$ & $<-0.1$ \\
\hline
\end{tabular}

Notes to Table 4 :

- LI-SMC 233 : Galaxy or PN ( 3 reference for each ...) ???

- LI-SMC 48 : near LHA 115-S 9 (B1, V=14.0), and RAW 479 (C;, V=16.3)

- LI-LMC 197: near the G4Ia supergiant HD 268759 but only very rough coordinates are available

- LI-LMC 203 : near SK -69 39a (A3Iab, B=12.5) and HV 12501 (M1.5, V=11.9)

- LI-LMC 493 : near BMB-BW49=BM 16.24 (C, I=14.0)

- LI-LMC 530 : near SHV0510004-692755 (M6, I=14.7, P=169)

- LI-LMC 568 : could be associated with IRAS-PSC 05112-6843

- LI-LMC 595 : neat BM 18-8 and 18-9 (C)

- LI-LMC 644 : near HV 2378

- LI-LMC 696: near BM 20-13 (C)

\begin{tabular}{|c|c|c|c|c|c|c|c|c|}
\hline LI & TRM & $\mathrm{RA}(1950)$ & $\operatorname{DEC}(1950)$ & $s_{12}$ & $S_{25}$ & $S_{60}$ & $\mathrm{C}_{21}$ & $\mathrm{C}_{32}$ \\
\hline 1197 & $\ldots$ & 053040 & .6645 & $0.11:$ & 0.22 & - & +0.0 & $<-0.1$ \\
\hline${ }^{*} 1198$ & $\ldots$ & 053040.3 & -703252 & 0.30 & 0.33 & - & -0.3 & \\
\hline$*_{1202}$ & $\ldots$ & 053059.5 & -680853 & $0.15:$ & $0.11:$ & C & -0.5 & - \\
\hline 1217 & $\ldots$ & 053115 & -6946 & 0.19 & 0.22 & 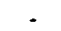 & -0.3 & $<-0.1$ \\
\hline 1227 & $\ldots$ & 053130 & $\begin{array}{l}-6759 \\
-6\end{array}$ & 0.15 & 0.11 : & 4.1 & -0.4 & +1.2 \\
\hline${ }^{*} 1243$ & $\ldots$ & 053152.3 & -724756 & $0.44:$ & & & $<-0.6$ & - \\
\hline 1245 & $\ldots$ & 053155 & -6806 & 0.22 & $0.11:$ & 2.5 & -0.6 & +1.0 \\
\hline$*_{1250}$ & $\ldots$ & 053201.6 & .702018 & 0.11 & 0.56 & $0.8:$ & +0.4 & -0.2 \\
\hline$*_{1278}$ & $\ldots$ & 053238.5 & .700419 & & 0.33 & & $>+0.0$ & $<-0.3$ \\
\hline 1283 & $\ldots$ & 053251 & -7113 & 0.26 & & $\mathrm{C}$ & $<-0.4$ & - \\
\hline$*_{1284}$ & $\ldots$ & 053252.5 & -682708 & 0.67 & 0.56 : & $\mathrm{C}$ & -0.4 & - \\
\hline 1313 & $\ldots$ & 053340 & .7033 & 0.26 & $0.11:$ & & -0.7 & $<+0.2$ \\
\hline 1316 & $\ldots$ & 053345 & -6944 & 0.19 & 0.22 & & -0.3 & $<-0.1$ \\
\hline 1339 & & 053440 & -7001 & 0.15 : & 0.11 : & - & -0.5 & $<+0.2$ \\
\hline & 031 & 053508.0 & .673222 & 0.21 & 0.24 & - & -0.3 & $<-0.1$ \\
\hline 1378 & $\ldots$ & 053550 & -7001 & 0.30 & 0.11 : & - & -0.8 & $<+0.2$ \\
\hline 1384 & & 053605 & .6904 & 0.22 & 0.22 & 4.1: & -0.3 & +0.9 \\
\hline 1386 & 145 & 053610 & -6637 & 0.26 & 0.22 & 5.0 & -0.4 & +1.0 \\
\hline$*^{*} 1424$ & & 0.53704 .5 & -701923 & 0.22 & 0.44 & 1.2 & +0.0 & +0.1 \\
\hline$*_{1435}$ & $\ldots$ & 053730.8 & -700221 & 0.33 & 0.33 & 4.1: & -0.3 & +0.7 \\
\hline 1454 & $\ldots$ & 053824 & -7116 & 0.19 & 0.17 & 1.7 & -0.4 & +0.6 \\
\hline${ }^{*} 1888$ & $\ldots$ & 053903.8 & -644913 & 0.37 & & & $<-0.5$ & - \\
\hline$*_{1502}$ & $\ldots$ & 054006.4 & .702006 & & 0.22 & & $>-0.2$ & $<-0.1$ \\
\hline 1505 & $\ldots$ & 054010 & -7110 & 0.22 & 0.22 & $\mathrm{C}$ & -0.3 & - \\
\hline${ }^{*} 1889$ & $\ldots$ & $05 \quad 4026.4$ & -645847 & 0.22 & & & -0.3 & - \\
\hline 1515 & $\ldots$ & 054030 & .6839 & 0.15 & 0.11 : & $\mathrm{C}$ & -0.5 & - \\
\hline 1526 & $\ldots$ & 054045 & .7034 & 0.15 & 0.44 & 1.2: & +0.1 & +0.1 \\
\hline$*_{1537}$ & $\cdots$ & 054101.3 & -652056 & 0.15 & 0.11 : & & -0.5 & $<+0.2$ \\
\hline$* 1540$ & $\ldots$ & 054105.1 & -695416 & 0.37 & & 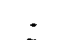 & -0.5 & - \\
\hline$*_{1570}$ & $\ldots$ & 054201.5 & .694333 & 0.37 & $0.33:$ & $\mathrm{C}$ & -0.4 & - \\
\hline 1604 & $\ldots$ & 054320 & .7032 & 0.11 & 0.11 : & - & -0.3 & $<+0.2$ \\
\hline$*^{*} 1630$ & $\ldots$ & 054411.7 & .685312 & 0.15 & $0.11:$ & 2.1: & -0.4 & +0.9 \\
\hline 1641 & $\ldots$ & 054430 & .7023 & 0.11 & 0.11 & 0.8 & -0.3 & +0.5 \\
\hline${ }^{*} 1646$ & $\ldots$ & $0544 \quad 40.6$ & -694525 & 0.37 & $0.33:$ & & -0.4 & $<-0.3$ \\
\hline 1652 & $\cdots$ & 054450 & -6840 & 0.11 & 0.11 : & 1.7 & -0.3 & +0.8 \\
\hline$*_{1654}$ & $\cdots$ & 054458.1 & -684915 & 0.22 & 0.22 & $\mathrm{C}$ & -0.3 & 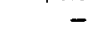 \\
\hline${ }^{*} 1657$ & $\ldots$ & $\begin{array}{lllll}05 & 45 & 03 & 0\end{array}$ & -703912 & 0.22 & $0.22:$ & 2.1 & -0.3 & +0.6 \\
\hline$*^{*} 1666$ & $\ldots$ & 054521.6 & -652205 & 0.15 & $0.56:$ & & +0.3 & $<-0.5$ \\
\hline 1692 & $\ldots$ & 054630 & -6940 & 0.48 & 0.22 & 8.3 & -0.7 & +1.2 \\
\hline 1700 & $\ldots$ & 054700 & -6926 & 0.19 & $0.11:$ & $\mathrm{C}$ & -0.6 & - \\
\hline$*_{1890}$ & $\cdots$ & 054712.7 & -643524 & 0.26 & & & -0.4 & . \\
\hline 1702 & $\ldots$ & 054714 & -7116 & 0.22 & $0.11:$ & - & -0.6 & $<+0.2$ \\
\hline 1703 & $\ldots$ & 054720 & .7015 & 0.11 & $0.06:$ & - & -0.6 & $<+0.5$ \\
\hline 1705 & $\ldots$ & 054724 & -7105 & 0.15 & $0.11:$ & $\mathrm{C}$ & -0.5 & - \\
\hline$*_{1712}$ & $\ldots$ & 054731.5 & -700414 & 0.22 & 0.22 : & 2.1 & -0.3 & +0.6 \\
\hline 1738 & $\ldots$ & 054850 & .6810 & 0.22 & 0.11 : & & -0.6 & $<+0.2$ \\
\hline$* 1745$ & $\ldots$ & 054934.3 & -703409 & 0.15 & 0.89 & 1.2 & +0.5 & -0.3 \\
\hline$*_{1757}$ & $\ldots$ & 055045.8 & -675108 & 0.15 & 0.22 & & -0.2 & $<-0.1$ \\
\hline$*_{1766}$ & $\cdots$ & $05 \quad 5215.3$ & .654523 & 0.11 : & 0.11 : & - & -0.3 & $<+0.2$ \\
\hline${ }^{*} 1768$ & $\ldots$ & 055215.9 & .712010 & 0.19 & 0.22 & & -0.3 & $<-0.1$ \\
\hline 1771 & $\ldots$ & 055310 & -6717 & 0.22 & $0.11:$ & $0.8:$ & -0.6 & +0.5 \\
\hline 1772 & $\ldots$ & 055315 & -68 & 0.22 : & 0.22 & 1.7 & -0.3 & +0.5 \\
\hline 1773 & $\ldots$ & 055340 & .7034 & 0.19 & $0.11:$ & & -0.6 & $<+0.2$ \\
\hline 1774 & $\ldots$ & 055342 & .6639 & & 0.22 & - & & $<-0.1$ \\
\hline 1775 & $\ldots$ & 055342 & -7137 & $0.44:$ & & & -0.6 & \\
\hline *1777 & $\ldots$ & 44.5 & -7 & 0.11 : & 0.56 & $0.4:$ & +0.4 & -0.5 \\
\hline *1780 & $\ldots$ & 055401.8 & -653337 & 0.3 & 0.22 & & -0.5 & $<-0.1$ \\
\hline$* 1784$ & $\ldots$ & 055443.2 & -651527 & 0.15 & 0.33 & - & +0.0 & $<-0.3$ \\
\hline$* 1785$ & $\ldots$ & 055506.2 & -652839 & & 0.22 & - & & \\
\hline *1795 & $\ldots$ & 055649.6 & -675354 & 0.33 & 0.44 & - & -0.2 & $<-0.4$ \\
\hline$*_{1803}$ & $\ldots$ & 055852.1 & & 0.1 & 0.56 & - & +0.2 & $<-0.5$ \\
\hline${ }^{*} 1807$ & $\ldots$ & 060108.9 & -663634 & 0.11 : & $0.22:$ & 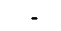 & +0.0 & $<-0.1$ \\
\hline$* 1813$ & $\ldots$ & 060235.3 & -671243 & $0.44:$ & $0.44:$ & - & -0.3 & $<-0.4$ \\
\hline & $\ldots$ & 060251.1 & -672215 & 0.67 & $0.33:$ & - & -0.6 & $<-0.3$ \\
\hline${ }^{*} 1818$ & $\ldots$ & 060307.4 & -72271 & 0.52 : & 0.5 & - & -0.3 & $<-0.5$ \\
\hline${ }^{*} 1821$ & 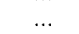 & 060432.6 & -672254 & 0.44 & $0.33:$ & - & -0.4 & $<-0.3$ \\
\hline
\end{tabular}

- LI-LMC 730: near BM 21-13 (C)

- LI-LMC 825 : could be associated with IRAS-PSC 05205-6913

- LI-LMC 987 ; near HD 269507 ( $K, B=11.5$ ) but only very rough coordinates are available. Could be associated with IRAS-PSC 05249-6916.

- LI-LMC 1028: near BM 23-21 (C)

- LI-LMC 1055: near SK -67 $109(\mathrm{~V}=13.1)$

- LI-LMC 1082: near SK - $6697(\mathrm{~B}=12.5)$ and SK $-6698(\mathrm{~B}=11.9)$

- LI-LMC 1378 : near BM 33-31 (C)

- LI-LMC 1657 : near BM 37-32 (C)

- LI-LMC 1775: near WORC 220 (C)

- LI-LMC 1785: AGN candidate in De Grijp et al. (1987)

- LI-LMC 1795: a bright $R$ counterpart is found by Zijlstra et al. (1995)

- LI-LMC 1807 = IRAS-PSC 06011-6636A 
Table 5. Unidentified sources from group 2

\begin{tabular}{|c|c|c|c|c|c|c|c|c|c|c|c|c|c|c|c|c|c|}
\hline LI & TRM & $\operatorname{RA}(1950)$ & $\operatorname{DEC}(1950)$ & $\mathrm{S}_{12}$ & $S_{25}$ & $S_{60}$ & $\mathrm{C}_{21}$ & $\mathrm{C}_{32}$ & LI & TRM & $\mathrm{RA}(1950)$ & $\operatorname{DEC}(1950)$ & $S_{12}$ & $\mathrm{~S}_{25}$ & $S_{60}$ & $\mathrm{C}_{21}$ & $\mathrm{C}_{32}$ \\
\hline$*_{221}$ & $\ldots$ & 001535.0 & .735610 & 0.19 & - & - & $<-0.3$ & - & $* 756$ & $\ldots$ & 051759.3 & -691837 & 0.41 & $\mathrm{C}$ & $\mathrm{C}$ & - & - \\
\hline$*_{226}$ & $\ldots$ & $00 \quad 18 \quad 05.8$ & -733730 & & - & 0.6 & - & $>+0.1$ & 791 & $\begin{array}{l}\cdots \\
\cdots\end{array}$ & 051900 & -7130 & 0.11 : & - & - & $<+0.0$ & - \\
\hline$* 227$ & $\cdots$ & 001849.3 & -745238 & $0.19:$ & - & 0.6 : & $<-0.3$ & $>+0.1$ & $* 794$ & $\ldots$ & 051910 & .6937 & - & 0.44 & $\mathrm{C}$ & $>+0.1$ & - \\
\hline$* 234$ & $\ldots$ & 002628.0 & -743747 & - & - & 0.6 & - & $>+0.1$ & $* 810$ & $\ldots$ & $05 \quad 1936.4$ & .692321 & 0.52 & 0.56 & $\mathrm{C}$ & -0.3 & - \\
\hline 238 & $\ldots$ & 002805 & -7429 & 0.19 & - & - & $<-0.3$ & - & 832 & $\cdots$ & 052040 & -6940 & 0.15 & 0.33 & $\mathrm{C}$ & +0.0 & - \\
\hline *19 & $\ldots$ & 004101.0 & .733945 & 0.11 : & . & $\mathrm{C}$ & $<+0.0$ & - & 858 & $\ldots$ & 052130 & -7117 & 0.15 & - & . & $<-0.2$ & - \\
\hline *20 & $\cdots$ & $0041 \quad 10.0$ & $-7336 \mathbf{3 5}$ & $0.11:$ & - & $\mathrm{C}$ & $<+0.0$ & - & 862 & $\ldots$ & 052140 & .6645 & $0.15:$ & 0.33 & $\mathrm{C}$ & +0.0 & - \\
\hline *31 & $\ldots$ & 004347.1 & .733433 & 0.11 & 0.22 & C & +0.0 & - & 863 & $\cdots$ & 052140 & -7016 & 0.15 & 0.22 & $\mathrm{C}$ & -0.2 & - \\
\hline 72 & $\ldots$ & 004918 & -7327 & 0.19 & 0.22 & $\mathrm{C}$ & -0.3 & - & 865 & $\ldots$ & 052145 & .7014 & 0.15 & 0.22 & $\mathrm{C}$ & -0.2 & - \\
\hline 78 & $\ldots$ & 005009 & -7257 & 0.11 : & 0.22 & $\mathrm{C}$ & +0.0 & - & 889 & $\cdots$ & 052230 & -6633 & 0.19 & 0.22 & $\mathrm{C}$ & -0.3 & - \\
\hline 96 & $\ldots$ & 005204 & -7259 & $0.19:$ & - & C & $<-0.3$ & - & $* 917$ & $\ldots$ & $\begin{array}{lll}05 & 23 & 12.4\end{array}$ & -694148 & $0.19:$ & 0.22 & $\mathrm{C}$ & -0.3 & - \\
\hline 97 & $\ldots$ & 005206 & -7300 & 0.19 & $0.22:$ & C & -0.3 & - & 975 & $\ldots$ & 052445 & -6903 & $0.15:$ & $0.22:$ & $\mathrm{C}$ & -0.2 & - \\
\hline 100 & $\ldots$ & 005221 & .7338 & $0.11:$ & 0.44 & C & +0.3 & - & 981 & $\ldots$ & 052450 & -7201 & 0.19 & - & - & $<-0.3$ & - \\
\hline 106 & $\ldots$ & 005346 & -7258 & 0.19 & 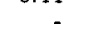 & $\mathrm{C}$ & $<-0.3$ & - & *993 & $\ldots$ & 052510.5 & -695301 & 0.15 & - & $\mathrm{C}$ & $<-0.2$ & - \\
\hline 112 & $\ldots$ & 005432 & -7323 & - & 0.22 & $\mathrm{C}$ & $>-0.2$ & - & *998 & $\ldots$ & $05 \quad 2518.4$ & -690816 & 0.15 & $\mathrm{C}$ & C & - & - \\
\hline 116 & $\ldots$ & 005503 & .7256 & . & 0.22 & $\mathrm{C}$ & $>-0.2$ & - & ${ }^{*} 1002$ & $\ldots$ & 052523.1 & -661857 & 0.93 & 1.78: & C & +0.0 & - \\
\hline 120 & $\ldots$ & 005530 & .7302 & $0.11:$ & 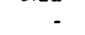 & $\mathrm{C}$ & $<0.0$ & - & ${ }^{*} 1010$ & $\ldots$ & 052540 & .6615 & 0.81 & 0.89 & $\mathrm{C}$ & -0.3 & - \\
\hline 122 & $\ldots$ & 005557 & -7310 & 0.19 & 0.22 & $\mathrm{C}$ & -0.3 & - & 1057 & $\begin{array}{l}\cdots \\
\ldots\end{array}$ & 052640 & .6924 & 0.22 & 0.33 & $\mathrm{C}$ & -0.1 & - \\
\hline 127 & $\ldots$ & 005640 & -7227 & $0.19:$ & 1.44: & C & +0.6 & - & $\ldots$ & 096 & 052652.2 & -662329 & 0.12 & 0.08 & - & -0.5 & $<+0.3$ \\
\hline 133 & $\ldots$ & 005742 & -7231 & 0.19 & $0.44:$ & $\mathrm{C}$ & +0.0 & - & ${ }^{*} 1094$ & $\ldots$ & 052750 & -7127 & 0.33 & 0.78 & $\mathrm{C}$ & +0.1 & - \\
\hline 143 & $\ldots$ & 005925 & -7204 & - & 0.22 & $\mathrm{C}$ & $>-0.2$ & - & 1116 & 114 & 052835 & .6730 & 0.15 & 0.22 & $\mathrm{C}$ & -0.2 & - \\
\hline 150 & $\ldots$ & 010027 & -7417 & $0.19:$ & 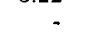 & - & $<-0.3$ & - & 1139 & $\ldots$ & 052910 & -6904 & $0.15:$ & 0.44 & C & +0.1 & - \\
\hline${ }^{*} 213$ & $\ldots$ & 012137.9 & -745050 & - & - & $0.4:$ & - & $>-0.1$ & 1147 & $\ldots$ & 052920 & -7016 & 0.11 & $\dot{0}$ & $\dot{s}$ & $<+0.0$ & - \\
\hline & & & & & & & & & $\begin{array}{l}{ }^{*} 1150 \\
* 1150\end{array}$ & $\cdots$ & $\begin{array}{l}052921.4 \\
05\end{array}$ & -691157 & $\begin{array}{l}0.15: \\
0.19\end{array}$ & 0.22 & $\mathrm{C}$ & $\begin{array}{l}-0.2 \\
-0.3\end{array}$ & - \\
\hline $\begin{array}{l}{ }^{*} 1830 \\
* 1832\end{array}$ & $\ldots$ & $\begin{array}{l}043142.0 \\
043229.5\end{array}$ & 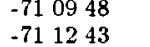 & $\dot{-}$ & $\dot{-}$ & $\begin{array}{l}0.4 \\
0.6\end{array}$ & - & $\begin{array}{l}>-0.1 \\
>+0.1\end{array}$ & $\begin{array}{l}{ }^{*} 1159 \\
{ }^{*} 1242\end{array}$ & $\begin{array}{l}\cdots \\
\cdots\end{array}$ & $\begin{array}{l}052942.9 \\
053151.7\end{array}$ & $\begin{array}{l}-651714 \\
-682436\end{array}$ & $\begin{array}{l}0.19: \\
0.22:\end{array}$ & $0 . \overline{33}$ & $\overline{\mathrm{C}}$ & $\begin{array}{l}-0.3 \\
-0.1\end{array}$ & $\overline{-}$ \\
\hline *1836 & $\begin{array}{l}\cdots \\
\cdots\end{array}$ & 043334.0 & -713706 & - & - & 0.4 & - & $>-0.1$ & 1246 & $\ldots$ & 053155 & -7104 & 0.37 & 0.44 & C & -0.2 & - \\
\hline *1839 & $\ldots$ & 043531.3 & -690958 & - & - & 0.4 & - & $>-0.1$ & 1257 & $\ldots$ & 053210 & -6900 & 0.15 & 0.33 & $\mathrm{C}$ & +0.0 & - \\
\hline${ }^{*} 1852$ & $\ldots$ & 044054.2 & -645455 & - & - & 0.6 & - & $>+0.1$ & $*^{*} 1292$ & 021 & 053300.9 & -674318 & 1.18 & 4.22 & $\mathrm{C}$ & +0.2 & - \\
\hline *1855 & $\ldots$ & 044218.3 & -650603 & - & . & 0.6 & - & $>+0.1$ & 1297 & $\ldots$ & 053315 & -7011 & 0.15 & - & - & $<-0.2$ & - \\
\hline 117 & $\ldots$ & 045245 & -6919 & 0.26 & 0.33 & $\mathrm{C}$ & -0.2 & - & ${ }^{*} 1315$ & 039 & 053345 & -6727 & 0.19 & 0.33 & $\mathrm{C}$ & -0.1 & - \\
\hline 120 & $\ldots$ & 045300 & -6812 & 0.19 & 0.22 & $\mathrm{C}$ & -0.3 & - & 1322 & $\ldots$ & 053355 & -6926 & 0.19 & 0.22 & $\mathrm{C}$ & -0.3 & - \\
\hline 125 & $\ldots$ & 045310 & -6932 & 0.19 & 0.22 & C & -0.3 & - & 1336 & $\ldots$ & 053435 & -6901 & 0.22 & 0.44 & $\mathrm{C}$ & +0.0 & - \\
\hline${ }^{*} 167$ & $\ldots$ & 045450.2 & .693114 & $0.07:$ & 0.11 : & C & -0.1 & - & 1350 & $\ldots$ & 053500 & -6824 & 0.19 & 0.22 & C & -0.3 & - \\
\hline 170 & $\ldots$ & 045500 & .7024 & 0.19 & 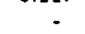 & . & $<-0.3$ & - & $* 1353$ & $\ldots$ & 053506.9 & -694348 & 0.19 & 0.22 & $\mathrm{C}$ & -0.3 & - \\
\hline 173 & $\ldots$ & 045505 & -6919 & 0.30 & 0.33 & C & -0.3 & - & ${ }^{*} 1886$ & $\ldots$ & 053536.8 & .650839 & - & - & 0.6 & - & $>+0.1$ \\
\hline$*_{177}$ & $\ldots$ & 045513.6 & -663614 & $0.11:$ & 0.78 & $\mathrm{C}$ & +0.5 & - & ${ }^{*} 1371$ & $\ldots$ & 053539.7 & -695440 & - & 0.11 : & $\mathrm{C}$ & $>-0.5$ & - \\
\hline 185 & $\ldots$ & 045525 & -6657 & 0.19 & & . & $<-0.3$ & - & 1375 & $\ldots$ & 053540 & -7013 & 0.15 & 0.22 & $\mathrm{C}$ & -0.2 & - \\
\hline 187 & $\ldots$ & 045530 & -6828 & 0.37 & 0.67 & $\mathrm{C}$ & -0.1 & - & 1390 & $\ldots$ & 053610 & -7003 & 0.15 & 0.33 & C & +0.0 & - \\
\hline$* 205$ & $\ldots$ & 045617.0 & -664140 & 0.19 & 0.56 & $\mathrm{C}$ & +0.2 & - & ${ }^{*} 1408$ & $\ldots$ & 053643.6 & -662609 & 0.22 & 0.56 & C & +0.1 & - \\
\hline$*_{210}$ & $\ldots$ & 045624.3 & -662948 & $1.48:$ & 10.55: & $\mathrm{C}$ & +0.5 & - & ${ }^{*} 1887$ & $\ldots$ & 053711.4 & -650549 & . & - & 0.4 & - & $>-0.1$ \\
\hline${ }^{*} 219$ & $\ldots$ & 045648.1 & .663534 & & 1.11: & $\mathrm{C}$ & $>+0.6$ & - & $* 1434$ & $\ldots$ & 053730 & -6950 & 0.56 & 0.78 & $\mathrm{C}$ & -0.2 & - \\
\hline$*_{229}$ & $\ldots$ & 045720.6 & -662352 & 0.37 & 0.56 & $\mathrm{C}$ & -0.1 & - & 1443 & $\ldots$ & 053750 & -6838 & 0.15 & 0.22 & $\mathrm{C}$ & -0.2 & - \\
\hline${ }^{*} 262$ & $\ldots$ & 045829.5 & .682837 & $0.11:$ & C & $\mathrm{C}$ & - & - & ${ }^{*} 1451$ & $\ldots$ & $0538 \quad 17.7$ & .693607 & 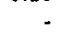 & 0.33 & $\mathrm{C}$ & $>+0.0$ & - \\
\hline${ }^{*} 266$ & $\ldots$ & 045839.1 & -661417 & 0.37 & 0.89 & $\mathrm{C}$ & +0.1 & - & 1461 & $\ldots$ & 053840 & -6933 & 0.44 & 1.11: & $\mathrm{C}$ & +0.1 & - \\
\hline$*_{271}$ & $\ldots$ & 045846.2 & -661137 & 0.30 & 0.33 & $\mathrm{C}$ & -0.3 & - & ${ }^{*} 1472$ & $\ldots$ & 053857.8 & -685459 & $0.19:$ & $0.22:$ & $\mathrm{C}$ & -0.3 & - \\
\hline$*_{281}$ & $\ldots$ & 045902.4 & -692148 & 0.15 & 0.33 & $\mathrm{C}$ & +0.0 & - & 1475 & $\ldots$ & 053900 & .7020 & 0.15 & 0.22 & $\mathrm{C}$ & -0.2 & - \\
\hline${ }^{*} 282$ & $\ldots$ & 045905.3 & -682937 & $0.15:$ & . & $\mathrm{C}$ & $<-0.2$ & - & *1485 & $\ldots$ & 053921.5 & .693616 & $0.37:$ & $\mathrm{C}$ & $\mathrm{C}$ & - & - \\
\hline$\ldots$ & 137 & 050309.6 & -671840 & - & 0.11 & $\mathrm{C}$ & $>-0.5$ & - & ${ }^{*} 1486$ & $\ldots$ & 053927.2 & -701514 & 0.26 & 0.33 & $\mathrm{C}$ & -0.2 & - \\
\hline 368 & $\ldots$ & 050340 & .6835 & 0.19 & 0.33 & $\mathrm{C}$ & -0.1 & - & $*_{1494}$ & $\ldots$ & 053950 & -6908 & 2.96 & 11.10: & $\mathrm{C}$ & +0.3 & - \\
\hline$* 375$ & 117 & 050357.1 & -672437 & 0.37 & 1.22 & $\mathrm{C}$ & +0.2 & - & 1516 & $\ldots$ & 054030 & -6908 & 0.93 & $2.77:$ & $\mathrm{C}$ & +0.2 & - \\
\hline *1875 & $\ldots$ & 050410.9 & .643324 & - & . & 0.6 & - & $>+0.1$ & $*^{*} 1520$ & $\ldots$ & 054035.2 & -693547 & $0.37:$ & 1.11: & $\mathrm{C}$ & +0.2 & - \\
\hline *385 & $\ldots$ & 050416.8 & -711108 & 0.07 : & 0.33 & $\mathrm{C}$ & +0.4 & - & 1535 & $\ldots$ & 054100 & -6854 & 0.30 & $0.56:$ & $\mathrm{C}$ & +0.0 & - \\
\hline 389 & $\cdots$ & 050430 & -6912 & 0.11 & 0.22 : & $\mathrm{C}$ & +0.0 & - & 1549 & $\ldots$ & 054115 & -6947 & 0.74 & 1.11 & $\mathrm{C}$ & -0.1 & - \\
\hline 391 & & 050440 & -6808 & 0.19 & 0.22 & $\mathrm{C}$ & -0.3 & - & ${ }^{*} 1552$ & $\ldots$ & 054122.2 & -693837 & 0.37 & $0.56:$ & $\mathrm{C}$ & -0.1 & - \\
\hline & 007 & $\begin{array}{llll}05 & 05 & 09.9\end{array}$ & -675137 & 0.15 & - & - & $<-0.2$ & - & 1564 & $\ldots$ & 054145 & -6933 & 0.15 & 0.33 & $\mathrm{C}$ & +0.0 & - \\
\hline$* 408$ & $\ldots$ & $\begin{array}{llll}05 & 05 & 10.1\end{array}$ & -675844 & 0.15 : & - & - & $<-0.2$ & - & 1569 & $\ldots$ & 054200 & -7039 & 0.19 & - & - & -0.3 & - \\
\hline 435 & $\ldots$ & 050615 & -6809 & 0.22 & 0.33 & $\mathrm{C}$ & -0.1 & - & 1573 & $\ldots$ & 054215 & .6859 & 0.44 & 1.00 & $\mathrm{C}$ & +0.0 & - \\
\hline 476 & $\ldots$ & 050800 & .7104 & $0.11:$ & & - & $<+0.0$ & - & 1603 & $\ldots$ & 054320 & .6917 & 0.22 & C & $\mathrm{C}$ & - & - \\
\hline 529 & $\ldots$ & 051000 & -6846 & 0.19 & 0.33 & $\mathrm{C}$ & -0.1 & - & 1617 & $\ldots$ & 054345 & -7113 & 0.15 & $0.06:$ & - & -0.7 & $<+0.5$ \\
\hline *531 & $\ldots$ & 051000.0 & -685004 & 0.33 & $0.67:$ & $\mathrm{C}$ & +0.0 & - & 1635 & $\ldots$ & 054418 & -6622 & 0.22 & $0.33:$ & $\mathrm{C}$ & -0.1 & - \\
\hline$* 551$ & $\ldots$ & 051044.2 & -693007 & 0.30 & 0.67 & $\mathrm{C}$ & +0.0 & - & 1642 & $\ldots$ & 054435 & -6849 & 0.19 & 0.33 & $C$ & -0.1 & - \\
\hline${ }^{*} 564$ & 140 & 051100.5 & -671125 & $0.07:$ & 0.28 & $\mathrm{C}$ & +0.3 & - & $*_{1671}$ & $\ldots$ & $05 \quad 45 \quad 44.2$ & -692227 & 0.15 & 0.33 & $\mathrm{C}$ & +0.0 & - \\
\hline 566 & $\ldots$ & 0.51105 & -6903 & 0.19 & 0.33 & $\mathrm{C}$ & -0.1 & - & 1681 & $\ldots$ & 054600 & -6932 & 0.19 & 0.22 : & C & -0.3 & - \\
\hline$*^{*} 611$ & $\ldots$ & $05 \quad 1245.8$ & -691123 & . & 0.33 & $\mathrm{C}$ & $>+0.0$ & - & ${ }^{*} 1740$ & $\ldots$ & 054857.6 & -700229 & 0.30 & 2.44 & $\mathrm{C}$ & +0.6 & - \\
\hline *1881 & $\ldots$ & $05 \quad 1258.2$ & -650328 & - & & 0.4 & - & $>-0.1$ & ${ }^{*} 1743$ & $\ldots$ & 054906.2 & -700624 & 0.78 & 2.22 & $\mathrm{C}$ & +0.1 & - \\
\hline 654 & $\ldots$ & 051415 & -6917 & 0.15 & 0.22 & $\mathrm{C}$ & -0.2 & -1 & ${ }^{*} 1758$ & $\ldots$ & 055053.8 & .714643 & $0.11:$ & - & - & $<+0.0$ & - \\
\hline 656 & $\ldots$ & 051420 & -6734 & 0.26 & 0.33 & $\mathrm{C}$ & -0.2 & - & 1765 & $\ldots$ & 0551.54 & .7107 & 0.15 & - & - & $<-0.2$ & - \\
\hline$*^{*} 679$ & $\ldots$ & 051525.8 & -692202 & 0.15 & 0.22 & $\mathrm{C}$ & -0.2 & - & $* 1770$ & $\ldots$ & 055238.8 & -652034 & $0.15:$ & - & - & $<-0.2$ & - \\
\hline 711 & $\ldots$ & 051630 & -6920 & 0.15 & 0.33 & $\mathrm{C}$ & +0.0 & - & *1798 & $\ldots$ & 055651.6 & -652839 & $0.15:$ & - & - & $<-0.2$ & - \\
\hline 718 & $\ldots$ & 051640 & -6818 & 0.11 & 0.22 & C & +0.0 & - & $* 1810$ & $\ldots$ & 060217.1 & -674303 & . & $0.11:$ & - & $>-0.5$ & $<+0.2$ \\
\hline *725 & $\ldots$ & 051700.0 & -693040 & 0.19 & - & - & -0.3 & - & *1822 & $\ldots$ & 060447.0 & -673659 & $0.15:$ & - & - & $<-0.2$ & - \\
\hline 740 & . & 051730 & -6942 & 0.15 & 0.22 & C & -0.2 & - & & & & & & & & & \\
\hline
\end{tabular}


Notes to Table 5 :

- LI-SMC 19 : near RAW 179 (C, V=16.8)

- LI-SMC 72 : near RAW 651 (C, V=17.3), RAW 658 (C, V=17.6), and the cepheid HV $1522(\mathrm{~V}=14.6)$

- LI-SMC 78 : in a cluster? Near RAW $706(\mathrm{C}, \mathrm{V}=17.0)$

- LI-SMC 96 : 96 and 97 are probably the same IRAS source. Near RAW 822 (C, V=17.6), AzV 148 (Bo, V=14.3), and the Cepheid HV 1598(V=15.9)

- LI-SMC 100: near RAW 832 (C, V=17.3)

- LI-SMC 106: near RAW 941 (C, V=17), the Cepheid HV $1649(\mathrm{~V}=15.5)$, and the foreground red variable $\mathrm{Z}$ Tuc $(\mathrm{B}=13)$

- LI-SMC 112 : near the foreground star HV 5627 (F7V, V=9.5)

- LI-SMC 133 : near the known or suspected KM supergiants SkKM 187, SkKM 190, HV 11402, and PMMR 100 (M0.5, V=12.8)

- LI-SMC 143 : near RAW 1258 (C, V=16.9) and RAW 1254 (C, V=17.1)

- TRM 137 : near HD $268931(G, B=12.2)$ and HD $268933(F, B=12.2)$ but only very rough coordinates are available

- LI-LMC 1839: AGN candidate in De Grijp et al. (1987)

- LI-LMC 478: According to Israel \& Koorneef (1991), there is a M giant or supergiant near the IRAS position. However the association is doubtful

- LI-LMC 435 : near the cepheid HV 893

- LI-LMC 531 : near the M supergiant HV $5625(\mathrm{~V}=12.6)$ and the B5lab supergiant HD $269101(\mathrm{~V}=12.0)$

- LI-LMC 541 : near BMB-BW097 (M6, I=14.0)

- LI-LMC 718 : near BM 21-10=SP 38-16(C)

- LI-LMC $794=$ IRAS.PSC 05191-6936. Near the C star SHV0518595-693653

- LI-LMC 832 : near the M6 star SHV0520342-693911

- LI-LMC 1010 : near the IRAS extended structure X0525-662

- LI-LMC 1094: near the IRAS extended structure X0527-714

- LI-LMC 1292 : near a nebulosity

- LI-LMC 1315 : could be associated with IRAS-PSC 05338-6725

- LI-LMC 1336: near HD 269762 (B9Ia, V=11.4), HV 2677(M3/5, V=13.6), and the PN SMP 1.78

- LI-LMC 1390 : near BM 33-37 and BM 33-43 (C)

- LI-LMC 1434 : could be associated with IRAS-PSC 05375-6949

- LI-LMC 1494 : could be associated with IRAS-PSC 05399-6906

- LI-LMC 1535 : near BM 36-7 (C) 
Table 6. Foreground stars

\begin{tabular}{|c|c|c|c|c|c|c|c|c|c|c|c|}
\hline LI & TRM & $\mathrm{RA}(1950)$ & $\operatorname{DEC}(1950)$ & $\mathrm{S}_{12}$ & $S_{25}$ & $S_{60}$ & $S_{100}$ & $\mathrm{C}_{2 \mathbf{1}}$ & $\mathrm{C}_{32}$ & group & identification \\
\hline 220 & $\ldots$ & 001458 & -7414 & $0.19:$ & 0.22 & - & - & -0.3 & $<-0.1$ & 1 & HD 1373 (KOIII, V=8.1) \\
\hline$*_{225}$ & $\ldots$ & 001635.2 & .741854 & 0.52 & 0.33 & - & - & -0.5 & $<-0.3$ & 1 & VV Tuc $(M 4, V=11)$ \\
\hline$*_{3}$ & $\ldots$ & 003347.8 & -740909 & 0.85 & 0.22 & 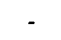 & $\mathrm{C}$ & -0.9 & $<-0.1$ & 1 & HD 3407 (M2III, V=8.7) \\
\hline$*_{4}$ & $\ldots$ & 003404.0 & .730803 & 0.44 & - & - & - & $<-0.6$ & - & 1 & HD $3439 ?(K 2 / 3, V=9.0)$ \\
\hline 7 & $\ldots$ & 003624 & .7414 & $0.11:$ & - & . & - & $<+0.0$ & - & 2 & $\mathrm{HD} 3689(\mathrm{~F} 6 \mathrm{~V}, \mathrm{~V}=7.4)$ \\
\hline 8 & $\ldots$ & 003644 & -7325 & $0.11:$ & . & - & $\mathrm{C}$ & $<+0.0$ & - & 2 & HD $3719(\mathrm{~A} 1 \mathrm{~m}, \mathrm{~V}=6.9)$ \\
\hline 13 & $\ldots$ & 004000 & -7358 & 0.19 & - & - & $\mathrm{c}$ & $<-0.3$ & - & 2 & HD $4090(\mathrm{~K} 1 / \mathrm{K} 2 \mathrm{III}, \mathrm{V}=9.2)$ \\
\hline$*_{22}$ & $\ldots$ & 004145.2 & .740029 & 0.44 & . & $\mathrm{C}$ & $\mathrm{C}$ & $<-0.6$ & - & 1 & HD $4252(\mathrm{~K} 3 / \mathrm{K} 4 \mathrm{III}, \mathrm{V}=8.8)$ \\
\hline 33 & $\ldots$ & 004432 & -7252 & 0.41 & - & - & - & $<-0.6$ & - & 1 & HD $4590(\mathrm{~K} 3 / \mathrm{K} 4 \mathrm{III}, \mathrm{V}=8.9)$ \\
\hline 56 & $\ldots$ & 004737 & -7345 & 0.44 & - & $\mathrm{C}$ & $\mathrm{c}$ & $<-0.6$ & - & 1 & HD $4893(\mathrm{~K} 2 \mathrm{III}, \mathrm{V}=8.4)$ \\
\hline 60 & $\ldots$ & 004803 & -7225 & $0.19:$ & - & - & - & $<-0.3$ & - & 2 & HD $4921(\mathrm{~K} 1 \mathrm{III}, \mathrm{V}=9.0)$ \\
\hline *69 & $\ldots$ & 004900.3 & -712536 & $0.11:$ & - & - & - & $<+0.0$ & - & 2 & HD $5028(\mathrm{~F} 5 \mathrm{~V}, \mathrm{~V}=6.9)$ \\
\hline 89 & $\ldots$ & Do 5123 & .7323 & 0.19 & - & $\mathrm{C}$ & $\mathrm{c}$ & $<-0.3$ & - & 2 & HD $5302(\mathrm{Ko}, \mathrm{V}=10)$ \\
\hline$* 90$ & $\ldots$ & 005124 & -7456 & 0.37 & - & - & - & $<-0.5$ & - & 1 & CF Tuc $(\mathrm{G} 3 \mathrm{~V}+?, \mathrm{~V}=7.6)$ \\
\hline$*_{101}$ & $\ldots$ & 005225.2 & -715326 & 0.48 & - & - & - & $<-0.7$ & - & 1 & PMMR $66(M 4, V=12.2)$ \\
\hline${ }^{*} 104$ & $\ldots$ & 005321.4 & -743435 & 0.67 & . & - & - & $<-0.8$ & - & 1 & HD $5499(\mathrm{~K} 1 \mathrm{IV}, \mathrm{V}=6.7)$ \\
\hline${ }^{*} 107$ & $\ldots$ & 005412.7 & -733443 & 1.15 & 0.56 & $\mathrm{C}$ & $\mathrm{C}$ & -0.6 & - & 1 & CM Tuc or PMMR $82(\mathrm{M} 4, \mathrm{~V}=12.5)$ \\
\hline$*^{*} 141$ & $\ldots$ & 005913.2 & .725817 & 0.67 & 0.17 & - & - & -0.9 & $<+0.0$ & 1 & HD $6172(\mathrm{~K} 2 / \mathrm{K} 3 \mathrm{III}, \mathrm{V}=7.7)$ \\
\hline$*_{145}$ & $\ldots$ & 005951.8 & .714903 & 0.33 & - & - & - & $<-0.5$ & - & 1 & HD 6222 (K1III, V=7.7) \\
\hline$*_{164}$ & $\ldots$ & 010227.8 & -734347 & 0.19 & - & - & - & $<-0.3$ & - & 2 & HD $6509(\mathrm{~K} 2 / \mathrm{K} 3 \mathrm{III}, \mathrm{V}=8.7)$ \\
\hline${ }^{*} 167$ & $\ldots$ & 010330.1 & -720008 & 0.44 & 0.17 & $\mathrm{C}$ & $\mathrm{C}$ & -0.7 & - & 1 & HD $6623(\mathrm{KOIII}, \mathrm{V}=7.4)$ \\
\hline${ }^{*} 171$ & $\ldots$ & 010348.5 & -711203 & $0.37:$ & & - & - & $<-0.5$ & - & 1 & HD $6662(\mathrm{G} 8 \mathrm{III}, \mathrm{V}=8.2)$ \\
\hline${ }^{*} 186$ & $\ldots$ & 010733.8 & .725439 & 2.59 & 0.78 & 0.4 & $\mathrm{C}$ & -0.8 & -0.7 & 1 & HD $7100(\mathrm{M} 3 / \mathrm{M} 4 \mathrm{III})$ \\
\hline${ }^{*} 191$ & $\ldots$ & 010927.6 & -715226 & 1.00 & $0.17:$ & - & & -1.1 & $<+0.0$ & 1 & PMMR $191(M 4, V=11.5)$ \\
\hline 196 & $\ldots$ & 011044 & -7411 & 0.19 : & - & - & - & $<-0.3$ & - & 2 & HD $7442(\mathrm{~F} 8 / \mathrm{GOV}, \mathrm{V}=7.2)$ \\
\hline$*_{198}$ & $\ldots$ & 011210.9 & .710807 & 0.30 & - & . & - & $<-0.5$ & - & 1 & $\mathrm{CPD}-7151(\mathrm{M} 0, \mathrm{~V}=9.4)$ \\
\hline 208 & $\ldots$ & 011606 & .7359 & 0.26 & $0.11:$ & $\mathrm{C}$ & $\mathrm{C}$ & -0.7 & - & 1 & PMMR $197(M 0, V=10.0)$ \\
\hline$*_{244}$ & $\ldots$ & 012907.7 & -732538 & 0.22 & 0.22 & - & - & -0.3 & $<-0.1$ & 1 & HD $9489(\mathrm{~K} 1 \mathrm{III}, \mathrm{V}=8.2)$ \\
\hline${ }^{*} 247$ & $\ldots$ & 013306.0 & .732100 & 0.19 & - & - & C & $<-0.3$ & - & 2 & PMMR $207(M 4, V=11.0)$ \\
\hline *1824 & $\ldots$ & 042723.0 & .710038 & 1.70 & 0.67 & - & - & -0.7 & $<-0.6$ & 1 & WOH G007 (M, I=6.1) \\
\hline 1828 & $\ldots$ & 043010 & -6759 & 0.19 & - & - & - & $<-0.3$ & - & 2 & HD $29137($ G5V,$V=7.7)$ \\
\hline$*_{1829}$ & $\ldots$ & 043135.2 & -722931 & 0.30 & 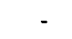 & - & - & $<-0.5$ & - & 1 & HD $29360($ KOIII, V=8.6) \\
\hline${ }^{*} 1831$ & $\ldots$ & 043216.6 & -650626 & 0.33 & $0.11:$ & - & - & -0.8 & $<+0.2$ & 1 & HD $29327($ K3III, V=8.4) \\
\hline 1833 & $\ldots$ & 043230 & -6521 & 0.15 & - & - & - & $<-0.2$ & - & 2 & HD $29339(\mathrm{G} 8, \mathrm{~V}=8.3)$ \\
\hline *1835 & $\ldots$ & 043303.7 & -672509 & 0.44 & $0.11:$ & . & - & -0.9 & $<+0.2$ & 1 & HD 29440 (K3III, V=8.7) \\
\hline${ }^{*} 1840$ & $\ldots$ & 043535.1 & -700803 & 0.81 & 0.22 & . & - & -0.9 & $<-0.1$ & 1 & WOH SG016 (M3, I=6.8) \\
\hline${ }^{*} 1842$ & $\ldots$ & 043708.5 & -702438 & 0.37 & 0.17 & - & - & -0.7 & $<+0.0$ & 1 & RT Men (Mira, $\mathrm{V}=12.2$ ) \\
\hline 1847 & $\ldots$ & 043815 & -6512 & 0.15 & - & - & - & $<-0.2$ & - & 2 & HD $29943(\mathrm{~K} 4 \mathrm{III}, \mathrm{V}=8.9)$ \\
\hline${ }^{*} 1848$ & $\ldots$ & 043903.3 & -693301 & 0.78 & 0.22 & - & - & -0.9 & $<-0.1$ & 1 & HD $30083(\mathrm{~K} 5 \mathrm{III}, \mathrm{V}=8.0)$ \\
\hline 1851 & $\ldots$ & 043930 & -6546 & 0.19 & - & - & - & $<-0.3$ & - & 2 & HD 30073 (K1III, V=8.1) \\
\hline${ }^{*} 1854$ & $\ldots$ & 044131.1 & -665946 & 0.37 & $0.11:$ & - & - & -0.8 & $<+0.2$ & 1 & HD $30325(\mathrm{~K} 2 / 3 \mathrm{III}, \mathrm{V}=8.1)$ \\
\hline${ }^{*} 7$ & $\ldots$ & 044141.6 & -684208 & 0.19 & - & - & - & $<-0.3$ & - & 2 & HD 30363 (K1III, V=7.8) \\
\hline 10 & $\ldots$ & 044300 & .7135 & $0.11:$ & - & - & - & $<+0.0$ & - & 2 & $\mathrm{HD} 30568$ (F6V, $\mathrm{V}=8.2)$ \\
\hline 14 & $\ldots$ & 044333 & .7101 & 0.15 & - & - & - & $<-0.2$ & - & 2 & HR 1541 (B8II/1II) \\
\hline$*_{17}$ & $\ldots$ & 044433.6 & -721335 & 0.33 & 0.11 : & . & - & -0.8 & $<+0.2$ & 1 & HD $30766(\mathrm{~K} 3 \mathrm{III}, \mathrm{V}=8.6)$ \\
\hline$*_{18} 18$ & $\ldots$ & 044503.0 & .704824 & 0.26 & $0.11:$ & - & - & -0.7 & $<+0.2$ & 1 & HV $12463(M, V=14.0)$ \\
\hline 19 & $\ldots$ & 044506 & .6829 & 0.19 & & - & - & $<-0.3$ & - & 2 & HD $270713(V=9.2)$ \\
\hline 20 & $\ldots$ & 044511 & .6807 & 0.19 & - & - & - & $<-0.3$ & - & 2 & HD $30759(\mathrm{~F} 6 / 7 \mathrm{~V}, \mathrm{~V}=8.1)$ \\
\hline 1864 & $\ldots$ & 044550 & .6610 & 0.19 & - & - & - & $<-0.3$ & - & 2 & HD $30805(F 3 V, V=7.4)$ \\
\hline 25 & $\ldots$ & 044646 & -6838 & 0.22 & . & - & . & $<-0.3$ & - & 1 & HD $30969(\mathrm{~F} 7 \mathrm{~V}, \mathrm{~V}=7.2)$ \\
\hline 30 & $\ldots$ & 044710 & -7112 & 0.19 & - & - & - & $<-0.3$ & - & 2 & $\mathrm{HD} 31080(\mathrm{G} 8 \mathrm{IV}, \mathrm{V}=8.1)$ \\
\hline 37 & $\ldots$ & 044808 & .6851 & 0.15 & - & 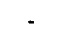 & . & $<-0.2$ & - & 2 & HD 31155 (F 3II/III, V=9.0) \\
\hline 1866 & $\ldots$ & 044830 & .6428 & 0.15 & - & - & - & $<-0.2$ & - & 2 & HD $31146(\mathrm{KOIII}, \mathrm{V}=8.6)$ \\
\hline$*_{50}$ & $\ldots$ & 044917.1 & .702035 & 0.26 & $0.11:$ & - & - & -0.7 & $<+0.2$ & 1 & HD $31335(\mathrm{KOIII}, \mathrm{V}=8.1)$ \\
\hline$*_{51}$ & $\ldots$ & 044920.3 & -665502 & 2.44 & 0.78 & - & - & -0.8 & $<-0.7$ & 1 & WY Dor (M4/5III, V=9.9) \\
\hline$* 78$ & $\ldots$ & 045104.0 & -695449 & 4.99 & 1.55 & $0.8:$ & - & -0.8 & -0.7 & 1 & HD $31518($ M3III, $\mathrm{V}=7.7)$ \\
\hline$* 87$ & $\ldots$ & 045128.0 & -680900 & 0.41 & 0.17 & - & - & -0.7 & $<+0.0$ & 1 & $\mathrm{HD} 31532(\mathrm{GOV}, \mathrm{V}=6.8)$ \\
\hline${ }^{*} 93$ & $\ldots$ & 045141.5 & -681038 & 0.44 & 0.17 & - & - & -0.7 & $<+0.0$ & 1 & HD $31576(\mathrm{~K} 4 \mathrm{II} / \mathrm{III}, \mathrm{V}=8.4)$ \\
\hline${ }^{*} 130$ & $\ldots$ & 045325.2 & -664522 & 4.33 & 1.55 & - & - & -0.8 & $<-1.0$ & 1 & HR 1598 (M0.5III, V=6.4) \\
\hline${ }^{*} 142$ & $\ldots$ & 045355.1 & .722920 & $0.30:$ & & - & - & $<-0.5$ & - & 1 & HR $1606(F 6 V, V=6.3)$ \\
\hline 147 & $\ldots$ & 045415 & -6722 & 0.74 & 0.33 & . & - & -0.7 & $<-0.3$ & 1 & HD 31907 (M1III) \\
\hline 1870 & $\ldots$ & 045550 & .6440 & 0.15 & - & - & . & $<-0.2$ & - & 2 & HD 32108 (G8IV, V=9.1) \\
\hline${ }^{*} 233$ & $\ldots$ & 045723.8 & -710002 & 0.44 & - & - & - & $<-0.6$ & - & 1 & HD 32415 (G8/KOIII, V=7.2) \\
\hline${ }^{*} 1871$ & $\ldots$ & 045733.0 & -644021 & 0.22 & 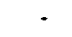 & 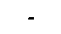 & 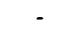 & $<-0.3$ & - & 1 & HD 32339 (K3III, V=9.1) \\
\hline 294 & $\ldots$ & 050003 & -6839 & $0.15:$ & & - & - & $<-0.2$ & - & 2 & HD $32762(\mathrm{~A} 5 \mathrm{III}, \mathrm{V}=8.0)$ \\
\hline${ }^{*} 1872$ & $\ldots$ & 050014.2 & -642747 & 0.89 & 0.17 & & & -1.0 & $<+0.0$ & 1 & HD $32714(\mathrm{~K} 1 / 2 \mathrm{III}, \mathrm{V}=7.3)$ \\
\hline${ }^{*} 321$ & $\ldots$ & 050139.1 & .680554 & 2.92 & 0.78 & - & - & -0.9 & $<-0.7$ & 1 & HD 32972 (M3III, $V=8.2$ ) \\
\hline${ }^{*} 324$ & $\ldots$ & 050141.4 & .681003 & 4.40 & 2.66 & - & 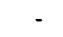 & -0.5 & $<-1.2$ & 1 & RX Dor $(M 7$, mira, $B=11.4)$ \\
\hline 327 & $\ldots$ & 050155 & .6934 & 0.19 & $0.11:$ & - & - & -0.6 & $<+0.2$ & 1 & HD $33031(A 7 \mathrm{~V}, \mathrm{~V}=8.1)$ \\
\hline
\end{tabular}


Table 6. continued

\begin{tabular}{|c|c|c|c|c|c|c|c|c|c|c|c|}
\hline LI & TRM & $\mathrm{RA}(1950)$ & $\operatorname{DEC}(1950)$ & $s_{12}$ & $\mathrm{~S}_{25}$ & $S_{60}$ & $S_{100}$ & $\mathrm{C}_{21}$ & $\mathrm{C}_{32}$ & group & identification \\
\hline$* 332$ & $\ldots$ & 050200.5 & -690322 & 0.41 & 0.33 & 1.2: & C & -0.4 & +0.2 & 1 & HD $33030(\mathrm{~K} 5 \mathrm{III}, \mathrm{V}=9.0)$ \\
\hline${ }^{*} 1873$ & $\ldots$ & 050257.0 & .643611 & 0.59 & 0.11 & - & - & -1.0 & $<+0.2$ & 1 & HD 33076 (MOIII, V=8.7) \\
\hline${ }^{*} 352$ & $\ldots$ & 050306.3 & -715435 & 0.15 & & - & - & $<-0.2$ & - & 2 & HD $33263(\mathrm{~K} 1 \mathrm{IV}, \mathrm{V}=8.3)$ \\
\hline *359 & $\ldots$ & 050321.0 & -712258 & 2.29 & 0.67 & - & - & -0.9 & $<-0.6$ & 1 & HR 1677 (G8III, V=5.3) \\
\hline${ }^{*} 1874$ & $\ldots$ & 050341.9 & -650445 & 4.81 & 1.44 & $0.2:$ & - & -0.8 & -1.2 & 1 & HD $33213(\mathrm{M} 4 \mathrm{III}, \mathrm{V}=8.1)$ \\
\hline 376 & $\ldots$ & 050400 & .7129 & 0.15 & - & - & - & $<-0.2$ & - & 2 & WOH SG136 (M0) \\
\hline 1876 & $\ldots$ & 050430 & -6437 & 0.19 & . & - & - & $<-0.3$ & - & 2 & HD $33293(\mathrm{KOIII}, \mathrm{V}=7.7)$ \\
\hline *406 & $\ldots$ & 050509.0 & .685811 & 0.15 & $0.11:$ & . & - & -0.5 & $<+0.2$ & 1 & HD $33477(\mathrm{~K} 1 \mathrm{III}, \mathrm{V}=8.0)$ \\
\hline 424 & $\ldots$ & 050548 & -7229 & 0.26 & & - & - & $<-0.4$ & - & 1 & HD $33652(\mathrm{KOIII}, \mathrm{V}=8.2)$ \\
\hline${ }^{*} 437$ & $\ldots$ & 050626.7 & .652626 & 0.30 & $0.11:$ & - & - & -0.8 & $<+0.2$ & 1 & HD $33616(\mathrm{~K} 2 \mathrm{III} / \mathrm{IV}, \mathrm{V}=7.8)$ \\
\hline$*_{470}$ & $\ldots$ & $\begin{array}{llll}0 & 07 & 44.2\end{array}$ & -711953 & 0.59 & 0.22 & - & - & -0.7 & $<-0.1$ & 1 & SAO 256161 (K2III) \\
\hline 500 & $\ldots$ & 050853 & -6714 & 0.22 & 0.11: & - & - & -0.6 & $<+0.2$ & 1 & HD $33986(\mathrm{~F} 5 \mathrm{~V}, \mathrm{~V}=9.1)$ \\
\hline 522 & & 050945 & -6913 & $0.15:$ & & - & - & $<-0.2$ & - & 2 & HD $34144(A 4 I I I / I V, V=9.4)$ \\
\hline 526 & $\ldots$ & 050950 & -6947 & $0.11:$ & & - & - & $<+0.0$ & - & 2 & HD $34170(\mathrm{~F} 6 \mathrm{~V}, \mathrm{~V}=8.4)$ \\
\hline$*_{532}$ & 091 & 051000.2 & -662903 & 0.78 & 0.22 & - & - & -0.9 & $<-0.1$ & 1 & HD 34127 (K5III, $\mathrm{V}=7.9)$ \\
\hline 554 & $\ldots$ & 051050 & -6923 & $0.11:$ & - & - & - & $<+0.0$ & - & 2 & HD 34276 (KIIII, V=8.7) \\
\hline${ }^{*} 565$ & $\ldots$ & 051101.4 & .720813 & 0.11 & - & - & - & $<+0.0$ & - & 2 & HD 34397 (G8/KOIII, $\mathrm{V}=7.9)$ \\
\hline${ }^{*} 585$ & $\ldots$ & 051149.7 & .693616 & 0.96 & 0.33 & 1.2 & - & -0.8 & +0.2 & 1 & HD $34437(\mathrm{~K} 5 \mathrm{III}, \mathrm{V}=7.6)$ \\
\hline 1879 & $\ldots$ & 051150 & .6514 & 0.19 & - & - & - & $<-0.3$ & - & 2 & HD $34349(F 5 \mathrm{~V}, \mathrm{~V}=7.0)$ \\
\hline${ }^{*} 639$ & $\ldots$ & 051347.3 & -671430 & 6.47 & 1.72 & - & - & -0.9 & $<-1.0$ & 1 & HR $1744(\mathrm{~K} 2.5 \mathrm{III}, \mathrm{V}=4.8)$ \\
\hline${ }^{*} 664$ & $\ldots$ & 051455.4 & .720557 & $0.22:$ & $0.17:$ & . & - & -0.4 & $<+0.0$ & 1 & WOH SG203 (MO+) \\
\hline 670 & $\ldots$ & 051500 & .7133 & $0.15:$ & - & - & - & $<-0.2$ & - & 2 & HD 34916 (KOIII, $\mathrm{V}=8.6)$ \\
\hline${ }^{*} 678$ & 110 & 051524.2 & .653548 & 4.07 & 2.00 & 1.2: & - & -0.6 & -0.6 & 1 & $\mathrm{HD} 271114(\mathrm{~F} 0, \mathrm{~B}=11.7)$ \\
\hline 714 & $\ldots$ & 051633 & -7031 & $0.11:$ & - & - & - & $<+0.0$ & - & 2 & HD $35095(\mathrm{~A} 4 / 5 \mathrm{IV} / \mathrm{V}, \mathrm{V}=8.6)$ \\
\hline$*_{732}$ & $\ldots$ & 051711.8 & .704842 & 0.26 & 0.17 & $0.4:$ & - & -0.5 & +0.0 & 1 & HV $928(\mathrm{M} 3, \mathrm{~V}=13.2)$ \\
\hline${ }^{*} 750$ & $\ldots$ & 051749.1 & -683840 & 0.26 & $0.06:$ & - & - & -1.0 & $<+0.5$ & 1 & HD $35230(G 8 I I I, V=7.6)$ \\
\hline 761 & $\ldots$ & 051805 & -6535 & 0.19 & - & - & - & $<-0.3$ & - & 2 & $\mathrm{HD} 271151(\mathrm{M} 0, \mathrm{~V}=9.4)$ \\
\hline${ }^{* 763}$ & $\ldots$ & 051812.4 & -724456 & 0.26 & . & - & - & $<-0.4$ & - & 1 & HD $35390($ K $4 \mathrm{III}, \mathrm{V}=\mathbf{8 . 3})$ \\
\hline 775 & 028 & 051830 & -6736 & 0.22 & $0.11:$ & - & - & -0.6 & $<+0.2$ & 1 & $(\mathrm{~V}=9.8)$ \\
\hline$* 800$ & 008 & 051923.6 & -675439 & 0.30 & $0.11:$ & - & - & -0.8 & $<+0.2$ & 1 & HD $269344(\mathrm{~K} 5 \mathrm{III}, \mathrm{V}=9.8)$ \\
\hline 814 & 090 & 051947 & -6630 & 0.26 & - & - & - & $<-0.4$ & - & 1,2 & HD 35461 (K1III, V=8.5) \\
\hline${ }^{* 838}$ & $\ldots$ & 052050 & .7101 & 0.19 & & - & - & $<-0.3$ & - & 2 & HD 35705 (KOIII, $V=8.3$ ) \\
\hline${ }^{*} 1883$ & $\ldots$ & 052050.6 & -645930 & 0.33 & 0.22 & - & - & -0.5 & $<-0.1$ & 1 & WOH G281 (M, V=12.5) \\
\hline 841 & $\ldots$ & 052100 & -6802 & 0.15 & $0.33:$ & $\mathrm{C}$ & $\mathrm{C}$ & +0.0 & - & 2 & HD $35665(\mathrm{KOIII} / \mathrm{IV}, \mathrm{V}=8.5)$ \\
\hline *871 & $\ldots$ & 052155.1 & -720827 & 0.30 & 0.17 & - & - & -0.6 & $<+0.0$ & 1 & HD $35906(\mathrm{~K} 4 \mathrm{III}, \mathrm{V}=8.5)$ \\
\hline 893 & $\ldots$ & 052240 & -6724 & 0.19 & $0.11:$ & - & - & -0.6 & $<+0.2$ & 1 & HD 35905 (KOIII, V=9.2) \\
\hline 954 & $\ldots$ & 052410 & -6933 & $0.11:$ & & - & - & $<+0.0$ & - & 2 & HD $269474(\mathrm{~V}=9.4)$ \\
\hline${ }^{*} 971$ & $\ldots$ & 052440.2 & -700349 & 1.22 & 0.33 & - & - & -0.9 & $<-0.3$ & 1 & HD $36241(\mathrm{M} 3 / 4, \mathrm{~V}=9.6)$ \\
\hline 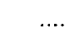 & 111 & 052505.8 & -675629 & 0.12 & - & - & - & $<-0.1$ & - & 2 & HD 36207 (K1III, V=9.0) \\
\hline & 013 & 052645.6 & -675037 & 0.13 & 0.09 & - & - & -0.5 & $<+0.3$ & 1 & $\mathrm{HD} 36347(\mathrm{G} 2 \mathrm{~V})$ \\
\hline 1075 & $\ldots$ & 052705 & -7231 & $0.19:$ & - & - & - & $<-0.3$ & - & 2 & HD $36637(\mathrm{G} 6 / 8 \mathrm{II} / \mathrm{III}, \mathrm{V}=9.7)$ \\
\hline$*^{*} 1077$ & $\ldots$ & 052706.6 & .700610 & 0.30 & - & - & - & $<-0.5$ & - & 1 & $\mathrm{HD} 36598(\mathrm{Kp} / \mathrm{C}, \mathrm{V}=8.1)$ \\
\hline 1081 & $\ldots$ & 052716 & .6840 & 0.44 & 0.22 & 1.2: & - & -0.6 & +0.4 & 1 & HD $36584($ Folv $/ \mathrm{V}, \mathrm{V}=6.0)$ \\
\hline 1095 & $\ldots$ & 052753 & .6807 & 0.11 & - & - & - & $<+0.0$ & - & 2 & HD $36650($ KOIII, V=8.8) \\
\hline$*_{* 1117}^{*}$ & 085 & $\begin{array}{l}052835.2 \\
052906.4\end{array}$ & $\begin{array}{l}-652912 \\
-664331\end{array}$ & $\begin{array}{l}0.59 ; \\
0.30\end{array}$ & $\begin{array}{l}0.17: \\
0.17\end{array}$ & 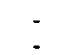 & : & $\begin{array}{l}-0.9 \\
-0.6\end{array}$ & $\begin{array}{l}<+0.0 \\
<+0.0\end{array}$ & $\begin{array}{l}1 \\
1\end{array}$ & HD $36805(\mathrm{~K} 2 \mathrm{III}, \mathrm{V}=8.1)$ \\
\hline 1174 & $\ldots$ & 053005 & .7018 & 0.19 & - & - & . & $<-0.3$ & - & 2 & HD $37084(\mathrm{~K} 2 / 3)$ \\
\hline${ }^{*} 1188$ & $\ldots$ & 053024.5 & .700023 & 0.30 & - & - & . & $<-0.5$ & - & 1 & HD $37122(\mathrm{~K} 2 / 3 \mathrm{III})$ \\
\hline${ }^{*} 1884$ & $\ldots$ & 053053.4 & -650925 & 0.30 & - & - & - & $<-0.5$ & - & 1 & HD 37093 (K5III) \\
\hline${ }^{*} 1266$ & 104 & 053225.5 & -655134 & 4.25 & 1.55 & - & - & -0.8 & $<-1.0$ & 1 & HD 37298 (M6III, V=10.1) \\
\hline${ }^{*} 1319$ & $\ldots$ & 053351.9 & -715942 & 0.70 & 0.22 & - & - & -0.8 & $<-0.1$ & 1 & HV $12830(M, V=12.4)$ \\
\hline${ }^{*} 1346$ & $\ldots$ & 053452.3 & -681412 & 0.41 & 0.22 & - & - & -0.6 & $<-0.1$ & 1 & HD $37668(\mathrm{~K} 2 \mathrm{III}, \mathrm{V}=8.0)$ \\
\hline${ }^{*} 1380$ & $\ldots$ & 053555.3 & -711001 & 1.04 & 0.22 & - & - & -1.0 & $<-0.1$ & 1 & HD $37899(\mathrm{~K} 3 \mathrm{HI}, \mathrm{V}=7.7)$ \\
\hline${ }^{*} 1385$ & & 053606.1 & -714221 & 0.33 & & - & - & $<-0.5$ & - & 1 & HD $37936(\mathrm{G} 8 \mathrm{III}, \mathrm{V}=8.2)$ \\
\hline${ }^{* 1395}$ & 098 & 053619.5 & -661909 & 1.59 & 0.44 & - & - & -0.9 & $<-0.4$ & 1 & SAO $249320(\mathrm{M} 4 \mathrm{III}, \mathrm{V}=8.7)$ \\
\hline 1418 & $\ldots$ & 053655 & -6635 & 0.30 & 0.22 & - & - & -0.5 & $<-0.1$ & 1 & HD $37935(B 9.5 e, v=6.3)$ \\
\hline${ }^{*} 1427$ & $\ldots$ & 053708.7 & -704515 & 0.26 & - & 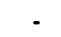 & & $<-0.4$ & - & 1 & HD $269838(G, B=11.3)$ \\
\hline 1476 & $\ldots$ & 053904 & .7141 & $0.11:$ & - & - & - & $<+0.0$ & - & 2 & HD $38330(\mathrm{G} 5 \mathrm{III} / \mathrm{IV}, \mathrm{V}=10.2)$ \\
\hline 1517 & $\ldots$ & 054030 & -7107 & 0.59 & 0.11 & - & - & -1.0 & +0.2 & 1 & \\
\hline 1530 & $\ldots$ & 054052 & -7230 & 0.19 & & - & - & $<-0.3$ & - & 2 & HD $3860 ?(\mathrm{~K} 21 \mathrm{II}, \mathrm{V}=8.6)$ \\
\hline${ }^{*} 1556$ & $\ldots$ & 31.4 & .72042 & 0.89 & 0.22 & 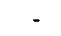 & - & -0.9 & $<-0.1$ & 1 & \\
\hline$*^{*} 1558$ & $\ldots$ & 054133.1 & -684738 & 0.89 & 0.44 & - & - & -0.6 & $<-0.4$ & 1 & HD 38617 (K3III, $\mathrm{V}=7.4)$ \\
\hline 1565 & $\ldots$ & 054149 & -6726 & 0.19 & & - & - & $<-0.3$ & - & 2 & HD $38616(\mathrm{~A} 2 \mathrm{Ib} / \mathrm{II}, \mathrm{V}=7.1)$ \\
\hline${ }^{*} 1566$ & $\ldots$ & 054150.2 & -685408 & $0.19:$ & $\mathrm{C}$ & $\mathrm{C}$ & $\mathrm{C}$ & - & - & 2 & $\mathrm{HD} 38654(\mathrm{M} 0 / 1, \mathrm{~V}=10.0)$ \\
\hline$*^{*} 1567$ & $\ldots$ & 054158.8 & -700320 & 2.96 & 0.78 & - & $\mathrm{C}$ & -0.9 & $<-0.7$ & 1 & HD $38706(\mathrm{M} 4 \mathrm{III}, \mathrm{V}=9.9)$ \\
\hline 1575 & $\ldots$ & 054220 & -6844 & 0.19 & $0.11:$ & - & - & -0.6 & $<+0.2$ & 1 & HD $38727(\mathrm{G} 5 \mathrm{III}, \mathrm{V}=8.5)$ \\
\hline 1587 & 055 & 054246 & .6710 & 0.15 & 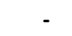 & - & - & $<-0.2$ & - & 2 & $\mathrm{HD} 38746(\mathrm{~F} 7 \mathrm{~V}, \mathrm{~V}=7.5)$ \\
\hline${ }^{*} 1616$ & $\ldots$ & 054343.5 & -682927 & 0.70 & 0.17 & 1.2: & 2.1: & -0.9 & +0.5 & 1 & $\mathrm{HD} 38922(\mathrm{M}, \mathrm{V}=10.4)$ \\
\hline${ }^{*} 1623$ & 019 & 054354.4 & -674310 & 8.18 & 4.55 & $0.8:$ & $\mathrm{C}$ & -0.6 & -1.1 & 1 & HD $38941(\mathrm{M} 5 / 6 \mathrm{III}, \mathrm{V}=9.3)$ \\
\hline${ }^{*} 1644$ & $\ldots$ & 054439.5 & -654519 & 1.22 & 0.33 & $0.4:$ & - & -0.9 & -0.3 & 1 & $\mathrm{HR} 2015(\mathrm{~A} 7 \mathrm{~V}, \mathrm{~V}=4.3)$ \\
\hline
\end{tabular}


Table 6. continued

\begin{tabular}{|c|c|c|c|c|c|c|c|c|c|c|c|}
\hline LI & TRM & RA(1950) & $\operatorname{DEC}(1950)$ & $\mathrm{S}_{12}$ & $\mathrm{~S}_{25}$ & $S_{60}$ & $S_{100}$ & $\mathrm{C}_{21}$ & $C_{32}$ & group & identification \\
\hline 1686 & $\ldots$ & 054615 & -6744 & $0.15:$ & - & - & . & $<-0.2$ & - & 2 & $\mathrm{HD} 39282(\mathrm{~A} 1 / 5 \mathrm{III}+\mathrm{F} 0, \mathrm{~V}=)$ \\
\hline$*_{1710}$ & $\ldots$ & 054731.0 & -674629 & 2.77 & 0.89 & - & - & -0.8 & $<-0.7$ & 1 & WOH SG500 (M4, I=2.9) \\
\hline 1725 & $\ldots$ & 054814 & -7100 & 0.15 & $0.11:$ & - & - & -0.5 & $<+0.2$ & 1 & HD $39675(\mathrm{~F} 5 \mathrm{~V}, \mathrm{~V}=8.9)$ \\
\hline 1728 & $\ldots$ & 054819 & -6639 & 0.22 & $0.11:$ & - & . & -0.6 & $<+0.2$ & 1 & HD 39580 (KoIII, V=7.9) \\
\hline${ }^{*} 1891$ & $\ldots$ & 054824.7 & -651054 & 0.44 & & - & - & $<-0.6$ & - & 1 & HD $39567(\mathrm{~K} 2 \mathrm{III}, \mathrm{V}=7.5)$ \\
\hline${ }^{*} 1731$ & $\ldots$ & 054826.6 & -694553 & 1.11 & $0.33:$ & - & - & -0.8 & -0.3 & 1 & HD 39674 (M3III, V=9.0) \\
\hline$*_{1736}$ & $\ldots$ & 054849.0 & .724304 & $0.78:$ & 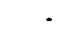 & - & - & $<-0.9$ & - & 1 & HR 2062 (KOIII, V=6.5) \\
\hline$*_{1748}$ & $\ldots$ & 054955.5 & -665453 & 0.26 & - & - & - & $<-0.4$ & - & 1 & HR $2064(\mathrm{~B} 6 \mathrm{~V}, \mathrm{~V}=5.1)$ \\
\hline$*_{1752}$ & $\ldots$ & 055024.1 & .694148 & $0.26:$ & $0.11:$ & - & - & -0.7 & $<+0.2$ & 1 & HD $39980($ K3III, V=8.6) \\
\hline${ }^{*} 1782$ & $\ldots$ & 055417.4 & -691455 & $0.11:$ & - & 0.4 & - & $<+0.0$ & $>-0.1$ & 2 & HD $40597(\mathrm{~K} 2 / 3 \mathrm{III}, \mathrm{V}=8.9)$ \\
\hline${ }^{*} 1788$ & $\ldots$ & 055538.0 & .673431 & 0.19 & - & - & - & $<-0.3$ & - & 2 & HD 40749 (K1III, V=8.1) \\
\hline${ }^{*} 1789$ & $\ldots$ & 055550.4 & .680315 & 0.44 & $0.11:$ & - & - & -0.9 & $<+0.2$ & 1 & HD $40810(\mathrm{~K} 2 \mathrm{III}, \mathrm{V}=7.5)$ \\
\hline${ }^{*} 1793$ & $\ldots$ & 055610.7 & -673251 & 0.26 & - & - & - & $<-0.4$ & - & 1 & HD $40845(\mathrm{~K} 2 / 3 \mathrm{III}, \mathrm{V}=8.4)$ \\
\hline${ }^{*} 1797$ & $\ldots$ & 055651.3 & .661842 & 0.11 : & - & - & - & $<+0.0$ & - & 2 & HD $40924(\mathrm{~K} 2 \mathrm{III}, \mathrm{V}=8.3)$ \\
\hline${ }^{*} 1800$ & $\ldots$ & 055712.5 & -700701 & 0.19 & - & - & . & $<-0.3$ & - & 2 & WOH SG523 (M) \\
\hline${ }^{*} 1802$ & $\ldots$ & 055830.9 & -690129 & $0.26:$ & - & - & - & $<-0.4$ & - & 1 & WOH SG524 (M2, I=7.4) \\
\hline${ }^{*} 1804$ & $\ldots$ & 055859.7 & -695129 & 0.81 & 0.17 & - & 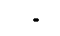 & -1.0 & $<+0.0$ & 1 & HD $41356(\mathrm{~K} 4 \mathrm{III}, \mathrm{V}=8.1)$ \\
\hline 1805 & $\ldots$ & 055903 & -7120 & $0.19:$ & - & - & - & $<-0.3$ & - & 2 & HD $41412(\mathrm{~K} 2 \mathrm{III}, \mathrm{V}=8.3)$ \\
\hline${ }^{*} 1809$ & $\ldots$ & 060214.3 & -700641 & 0.74 & 0.22 & - & . & -0.8 & $<-0.1$ & 1 & HD $41925(\mathrm{~K} 3 \mathrm{III} / \mathrm{IV}, \mathrm{V}=8.0)$ \\
\hline${ }^{*} 1811$ & $\ldots$ & 060225.4 & .703529 & 2.77: & 1.66: & - & - & -0.5 & $<-1.0$ & 1 & RU Men (Me, B=12.3) \\
\hline${ }^{*} 1812$ & $\ldots$ & 060225.5 & -664554 & 0.15 & - & . & - & $<-0.2$ & - & 2 & $\mathrm{OM} 88(\mathrm{~B} 3, \mathrm{~B}=10.5)$ \\
\hline$*_{1814}$ & $\ldots$ & 060238.2 & .720844 & 1.37: & $0.33:$ & - & - & -0.9 & $<-0.3$ & 1 & HD $42082($ MOIII, $\mathrm{V}=7.8)$ \\
\hline$*_{1815}$ & $\ldots$ & 060240.4 & .704022 & 1.55 & 0.56 & - & - & -0.8 & $<-0.5$ & 1 & $\mathrm{HD} 42030(\mathrm{M} 2 \mathrm{HI}, \mathrm{V}=8.5)$ \\
\hline 1816 & $\ldots$ & 060251 & -7103 & $0.15:$ & - & - & - & $<-0.2$ & - & 2 & HD $42080(F m, V=9.2)$ \\
\hline${ }^{*} 1820$ & & 060413.0 & -694222 & $0.89:$ & $0.56:$ & - & - & -0.5 & $<-0.5$ & 1 & NSV $2830($ M3III, V=9.0) \\
\hline
\end{tabular}

Notes to Table 6 : LI-SMC 90 could be identified with IRAS 00515-7455; LI-LMC 147 could be identified with IRAS 04544-6722; LI-LMC 838 could be identified with IRAS 05209-7101; LI-LMC $1812=$ IRAS 06024-6645A.

Table 7. Ruled out sources

\begin{tabular}{|c|c|c|c|c|c|c|c|c|c|c|c|}
\hline LI & TRM & $\mathrm{RA}(1950)$ & $\operatorname{DEC}(1950)$ & $\mathrm{S}_{12}$ & $S_{25}$ & $\mathrm{~S}_{60}$ & $S_{100}$ & $C_{21}$ & $\mathrm{C}_{32}$ & group & identification \\
\hline$*_{2}$ & $\ldots$ & 003343.2 & -733749 & 0.07 & 0.44 & 0.8 & 1.0 & +0.5 & -0.1 & 1 & AGN candidate \\
\hline 32 & $\ldots$ & 004351 & .7339 & 0.33 & 0.22 & C & $\mathrm{C}$ & -0.5 & - & 1 & LHA $115-\mathrm{N} 13(\mathrm{AB})$, HII region \\
\hline *36 & $\ldots$ & 004447.0 & .732229 & 0.52 & 1.78 & 21.0 & 42.0 & +0.2 & +0.7 & 3 & LHA $115-N$ 12A, HII region [5] \\
\hline${ }^{*} 54$ & $\ldots$ & 004726.9 & .733045 & 0.19 & $\mathrm{C}$ & $\mathrm{C}$ & $\mathrm{C}$ & - & - & 2 & SNR $0047-73.5$ \\
\hline 74 & $\ldots$ & 004930 & -7300 & 0.19 & 0.22 & $\mathrm{C}$ & $\mathrm{C}$ & -0.3 & - & 2 & Lindsay 41 , cluster \\
\hline$* \ldots$ & $\cdots$ & 005206.0 & -705421 & $0.25:$ & 0.84 & 1.11 & - & $+0.2:$ & - & 3 & galaxy [5] \\
\hline * 155 & $\ldots$ & 010119 & -7218 & 0.26 & 0.78 & $\mathrm{C}$ & C & +0.2 & - & 2 & $\mathrm{OB}$ assoc. \\
\hline $\begin{array}{l}* 156 \\
* 158\end{array}$ & $\ldots$ & 010131.0 & -722216 & 0.37 & 0.67 & C & $\mathrm{C}$ & -0.1 & - & 2 & OB assoc. \\
\hline $\begin{array}{r}158 \\
172\end{array}$ & $\begin{array}{l}\ldots \\
\ldots\end{array}$ & 010132.8 & -710659 & 0.67 & - & - & - & $<-0.8$ & - & 1 & NGC 362, cluster \\
\hline$* 173$ & $\cdots$ & 010350 & -7212 & - & 0.28 & 0.8 & $\mathrm{C}$ & $>+0.0$ & +0.1 & 1 & $\mathrm{AzV} 358 \mathrm{a}(\mathrm{B} 0)$ \\
\hline 182 & $\ldots$ & 010356.9 & -730559 & 0.19 & 1.00 & 3.7 & 6.3 & +0.4 & +0.2 & 3 & {$[5]$} \\
\hline $\begin{array}{l}102 \\
* 200\end{array}$ & $\cdots$ & 010641 & -7310 & 0.19 & - & $\mathrm{C}$ & $\mathrm{C}$ & $<-0.3$ & - & 2 & NGC 419 , cluster \\
\hline$* 200$ & $\ldots$ & 011241.2 & $\begin{array}{lll}-73 & 3242\end{array}$ & 0.70 & 4.00 & C & $\mathrm{C}$ & +0.4 & - & 2 & NGC 456 , cluster \\
\hline *201 & $\ldots$ & 011319.1 & -733342 & 0.30 & 2.00 & $\mathrm{C}$ & $\mathrm{C}$ & +0.5 & - & 2 & NGC 460 , cluster \\
\hline$*_{205}$ & $\ldots$ & 011418.1 & -732604 & - & 0.44 & C & $\mathrm{C}$ & $>+0.1$ & - & 2 & HW 72, cluster \\
\hline *1838 & $\ldots$ & 043514.5 & -664359 & - & $\cdot$ & 0.4 & - & - & -0.1 & 2 & ESO $84-23$, galaxy \\
\hline 63 & $\ldots$ & 044955 & .6917 & 0.48 & 1.44 & $\mathrm{C}$ & $\mathrm{C}$ & +0.2 & - & 2 & LHA $120-\mathrm{N} 77 \mathrm{E}, \mathrm{HIl}$ region \\
\hline${ }^{*} 67$ & $\ldots$ & 045029.8 & -693447 & 0.56 & 0.89 & 14.9 & 47.8 & -0.1 & +0.8 & 3 & {$[5]$} \\
\hline *138 & $\ldots$ & 045346.0 & -692236 & 0.22 & 1.11 & $\mathrm{C}$ & $\mathrm{C}$ & +0.4 & - & 2 & LHA $120-N 82$ (WC9) \\
\hline *162 & $\ldots$ & 045440.6 & -691539 & 1.41 & 12.76 & 41.4: & $\mathrm{C}$ & +0.6 & +0.1 & 1 & NGC 1748, HII region \\
\hline 182 & $\ldots$ & 045520 & -6925 & 0.30 & 0.22 & $\mathrm{C}$ & $\mathrm{C}$ & -0.5 & - & 1 & LHA $120-N 89$ \\
\hline 222 & $\ldots$ & 045700 & -6639 & 0.26 & $\mathrm{C}$ & $\mathrm{C}$ & $\mathrm{C}$ & - & - & 2 & HD 268732 (B1Ia, V $=11.6)$ \\
\hline${ }^{*} 225$ & $\ldots$ & 045708.5 & -695458 & 0.81 & 1.22 & 0.4 & - & -0.1 & -0.9 & 1 & HD 268835 (B8Ia, LBV) \\
\hline$* 226$ & $\ldots$ & 045709.2 & -662745 & 1.00 & 4.99: & $\mathrm{C}$ & $\mathrm{C}$ & +0.4 & - & 2 & LHA $120-\mathrm{N} 11 \mathrm{~A}$, HII region \\
\hline *235 & $\ldots$ & 045725.9 & -682936 & 2.81 & 12.9 & 118.0 & 228.4 & +0.3 & +0.6 & 3 & HD 268804 (B2Iab, V =11.2) [5] \\
\hline *276 & $\ldots$ & 045856.7 & -654738 & $0.07:$ & 0.44 & - & - & +0.5 & $<-0.4$ & 1 & LH 15 , cluster \\
\hline 283 & $\ldots$ & 045915 & .6617 & 0.11 & 0.11 & 1.7 & $\mathrm{C}$ & -0.3 & +0.8 & 1 & SK-66 $47(09, V=13.1)$ \\
\hline 309 & $\ldots$ & 050050 & -6600 & 0.07 & 0.22 & - & - & +0.2 & $<-0.1$ & 1 & HD $270948(0)$; X ray \\
\hline *346 & $\ldots$ & 050244.2 & -712415 & 0.81 & 8.88 & 5.0 & 2.1 & +0.7 & -0.6 & 1 & HD 269006 (LBV) \\
\hline 355 & $\ldots$ & 050315 & -6553 & 0.22 & 0.11 & - & - & -0.6 & $<+0.2$ & 1 & SK.65 $26(0)$ \\
\hline 379 & $\ldots$ & 050407 & -6631 & 0.19 & - & - & - & $<-0.3$ & - & 2 & NGC 1818 , cluster \\
\hline$\ldots$ & 113 & 050454.3 & -673606 & 0.13 & 0.13 & - & - & -0.3 & $<+0.1$ & 1 & LH 22, cluster \\
\hline 411 & $\ldots$ & 050515 & .6806 & 0.19 & 0.11 & $\mathrm{C}$ & $\mathrm{C}$ & -0.6 & - & 1 & CPD-68 $312(\mathrm{~B} 1, \mathrm{~V}=11.7)$ \\
\hline$* 423$ & $\ldots$ & 050546.4 & -675644 & $\cdot$ & 0.17 & 0.4 & - & $>-0.3$ & +0.0 & 1 & SNR0505-67.9 \\
\hline
\end{tabular}


Table 7. continued

\begin{tabular}{|c|c|c|c|c|c|c|c|c|c|c|c|}
\hline LI & TRM & $\mathbf{R A}(1950)$ & $\operatorname{DEC}(1950)$ & $s_{12}$ & $\mathrm{~S}_{25}$ & $\mathrm{~S}_{60}$ & $S_{300}$ & $\mathrm{C}_{21}$ & $\mathrm{C}_{32}$ & group & identification \\
\hline$*^{*} 510$ & $\ldots$ & 0.50916 .1 & -684815 & 0.52 & 1.00 & 14.5 & 45.8 & 0.0 & +0.8 & 3 & cluster [5] \\
\hline$*_{520}$ & $\ldots$ & 050938.6 & .684930 & 0.41 & 1.55 & $\mathrm{C}$ & $\mathrm{C}$ & +0.3 & - & 2 & LHA $120-\mathrm{N} 103 \mathrm{~A}$, HII region \\
\hline 573 & $\ldots$ & 051120 & -6857 & 0.26 & 0.44 & $\mathrm{C}$ & $\mathrm{C}$ & -0.1 & - & 2 & CSI-68-05114 (B8Ib) \\
\hline${ }^{*} 588$ & $\ldots$ & 051151.7 & -684717 & . & $0.11:$ & - & - & $>-0.5$ & $<+0.2$ & 2 & HD $269158(\mathrm{~A})$, NGC 1863 \\
\hline 594 & $\ldots$ & 051210 & -6715 & 0.26 & 0.44 & $\mathrm{C}$ & $\mathrm{C}$ & -0.1 & - & 2 & CSI-67-05122 1 and 2 (B9Ib, B9II) \\
\hline$*_{620}$ & $\ldots$ & 051300.2 & -702759 & 0.74 & 2.00 & 19.0 & 25.0 & +0.1 & +0.6 & 3 & LHA $120-\mathrm{N} 193 \mathrm{C}$, HII region [5] \\
\hline $\begin{array}{l}734 \\
736\end{array}$ & $\begin{array}{l}\ldots \\
\ldots\end{array}$ & $\begin{array}{l}051715 \\
051720\end{array}$ & $\begin{array}{l}-6856 \\
-6911\end{array}$ & $\begin{array}{l}0.15 \\
0.15\end{array}$ & $\begin{array}{l}0.11 \\
0.11\end{array}$ & 2.1: & $\mathrm{C}$ & $\begin{array}{l}-0.4 \\
-0.5\end{array}$ & $\begin{array}{r}+0.9 \\
<+0.2\end{array}$ & $\begin{array}{l}1 \\
1\end{array}$ & $\begin{array}{l}\text { cluster } \\
\text { BI } 122(B 111, V=13.1)\end{array}$ \\
\hline$* 772$ & $\ldots$ & 051828.7 & -693542 & 0.59 & 0.56 & 4.1: & $\mathrm{C}$ & -0.3 & +0.5 & 1 & cluster \\
\hline$* 816$ & $\ldots$ & 051948.4 & .694140 & 1.85 & 4.44 & $\mathrm{C}$ & $\mathrm{C}$ & +0.1 & - & 2 & HD 269382 (B5), HD 35517 (WC) \\
\hline$* 859$ & $\ldots$ & 0.52130 .7 & -670800 & $0.11:$ & 0.11 : & 1.2 & 2.1: & -0.3 & +0.7 & 1 & HD $269400(B 5, V=11.6)$ \\
\hline & 105 & 052134.9 & -654754 & 0.18 & - & - & - & $<-0.2$ & - & 2 & HD $271191(B)$, SK-65 $52(B 0)$ \\
\hline *866 & $\ldots$ & 052145.7 & .700154 & 0.56 & 0.78 & 9.1 & 18.7 & -0.2 & +0.7 & 3 & [5] \\
\hline$* 876$ & $\ldots$ & 052203.5 & -675816 & 0.56 : & 8.88 & 20.7: & $\mathrm{C}$ & +0.9 & +0.0 & 1 & NGC 1929 , cluster \\
\hline$* 887$ & $\ldots$ & 052223.7 & -680128 & 1.11: & 22.20 & $\mathrm{C}$ & $\mathrm{C}$ & +1.0 & - & 2 & HII region \\
\hline$* 908$ & $\ldots$ & 052301.3 & -685819 & 0.22 & 0.33 & - & - & -0.1 & $<-0.3$ & 1 & CSI-68-052302 $(\mathrm{A} 1 \mathrm{Ib}, \mathrm{B}=13.6)$ \\
\hline 913 & $\ldots$ & 052310 & -6648 & 0.15 & 0.22 & $\mathrm{C}$ & $\mathrm{c}$ & -0.2 & - & 2 & LMC-CO21, molec. cloud \\
\hline 926 & $\ldots$ & 052325 & -6712 & 0.07 & 0.11 & $\mathrm{C}$ & $\mathrm{C}$ & -0.1 & - & 2 & BI $153(\mathrm{~B} 2 \mathrm{II}, \mathrm{V}=13.6)$ \\
\hline 951 & $\ldots$ & 052406 & .7115 & 0.15 & 0.17 : & $\mathrm{C}$ & $\mathrm{C}$ & -0.3 & - & 2 & CSI-71-05240 1 (B9II, $B=13.0)$ \\
\hline${ }^{*} 961$ & $\ldots$ & 052426.9 & .683232 & 0.63 & 1.66 & 11.2 & $\mathrm{C}$ & +0.1 & +0.5 & 3 & LHA $120-\mathrm{N} 138 \mathrm{D}$, HII region [5] \\
\hline 985 & $\ldots$ & 052454 & -7137 & $0.26:$ & 0.33 : & $\mathrm{C}$ & $\mathrm{C}$ & -0.2 & - & 2 & HS 274 , cluster \\
\hline$\ldots$. & 128 & 052501.3 & .661457 & 0.15 & - & . & & $<-0.2$ & - & 2 & Brey $30(\mathrm{WR})$ \\
\hline & 037 & 0.52606 .9 & -673104 & 0.11 & . & - & - & $<0.0$ & - & 2 & BI $169(\mathrm{~B} 11 \mathrm{I}, \mathrm{V}=12.5)$, SK-67 101 (O9III, $\mathrm{V}=12.6)$ \\
\hline$*_{1100}$ & $\ldots$ & 052800.3 & -691025 & 3.88 & 23.31 & 10.3 & 4.2 & +0.5 & -0.7 & 1 & NGC 1984 , cluster \\
\hline 1112 & $\ldots$ & 052830 & .6743 & $0.11:$ & 0.11 : & 2.5 & 4.2 & -0.3 & +1.0 & 1 & $\mathrm{HD} 269593(\mathrm{~B} 5, \mathrm{~V}=11.4)$ \\
\hline$* 1126$ & $\ldots$ & 052842.1 & -661626 & 0.19 & - & - & - & $<-0.3$ & - & 2 & NGC 1978 \\
\hline${ }^{*} 1127$ & $\ldots$ & 052843.1 & -691059 & 1.11 & 1.44 & $\mathrm{C}$ & C & -0.2 & - & 2 & NGC 1994 7, cluster \\
\hline$* 1130$ & 099 & 052858.9 & -661741 & 0.11 & 0.44 & & & +0.3 & $<-0.4$ & 1 & {$[7]$} \\
\hline 1160 & $\ldots$ & 052945 & .7009 & 0.22 & 0.33 & $0.8:$ & $\mathrm{C}$ & -0.1 & +0.0 & 1 & BI 180 (B1II:) \\
\hline$*_{1199}$ & $\ldots$ & 053042.4 & .710715 & 0.52 & 1.33 & 24.8 & 52.0 & +0.1 & +0.9 & 3 & cluster [5] \\
\hline & 050 & 053043.1 & .671916 & 0.21 & 0.12 & - & & -0.6 & $<+0.2$ & 1 & cluster \\
\hline *1209 & $\ldots$ & 053103.1 & -691347 & 0.11 : & 0.33 : & $\mathrm{C}$ & $\mathrm{C}$ & +0.2 & - & 2 & HV $2605(\mathrm{Be})$ \\
\hline 1211 & $\ldots$ & 053105 & -6845 & 0.15 & $0.22:$ & $\mathrm{C}$ & $\mathrm{C}$ & -0.2 & - & 2 & SK-68 109 (A0Iab, V=12.5) \\
\hline 1215 & $\ldots$ & 053110 & .6908 & 0.11 & 0.33 & $\mathrm{C}$ & $\mathrm{C}$ & +0.2 & - & 2 & SK -69 $168(\mathrm{~B} 1.5, \mathrm{~V}=12.8)$ \\
\hline$*_{1239}$ & & 053145.3 & -690739 & . & 0.78 & $\mathrm{C}$ & $\mathrm{C}$ & $>+0.4$ & - & 2 & HD 269687 (Ofpe/WN9) \\
\hline${ }^{*} 1259$ & 112 & 053210.8 & -674430 & 1.48 & 5.99 & $\mathrm{C}$ & $\mathrm{C}$ & +0.3 & - & 2,1 & HII region (?) \\
\hline & 030 & 053227.7 & .673435 & 0.18 & 0.18 & - & - & -0.3 & $<+0.0$ & 1 & HD 269726 (B8Iab) \\
\hline *1274 & 022 & 053234.9 & .674341 & 1.78 & 8.88 & 113.3 & 280.8 & +0.4 & +0.7 & 3 & LHA $120-\mathrm{N} 57 \mathrm{~A}$, HII region \\
\hline * 1295 & & 053314.8 & .702529 & 0.15 : & - & - & - & $<-0.2$ & - & 2 & NOVA LMC 1981 \\
\hline${ }^{* 1317}$ & 149 & 053348.7 & .661729 & - & $0.11:$ & - & - & $>-0.5$ & $<+0.2$ & 2 & BI 206 (B1II) \\
\hline 1342 & $\ldots$ & 053445 & .6912 & 0.22 & 0.33 & $\mathrm{C}$ & $\mathrm{C}$ & -0.1 & - & 2 & SK-69 $192(B 1.5, V=12.8)$ \\
\hline$*_{1370}$ & $\ldots$ & 053535.2 & -691555 & & 1.33: & $\mathrm{C}$ & $\mathrm{C}$ & $>+0.6$ & - & 2 & Brey $56(\mathrm{WN}, \mathrm{V}=13.6)$, BI $227(\mathrm{O}, \mathrm{V}=13.7)$ \\
\hline${ }^{*} 1383$ & $\ldots$ & 053602.2 & .691422 & 1.48 & 5.55 & 124.2 & 228.8 & +0.2 & +1.0 & 3 & Brey $58(W N)$, nebula [5] \\
\hline 1387 & $\ldots$ & 053610 & .6732 & 0.15 & $0.22:$ & $\mathrm{C}$ & $\mathrm{C}$ & -0.2 & - & 2 & SK-67 221 (V=12.9) \\
\hline${ }^{*} 1406$ & $\ldots$ & 053638.0 & .694300 & 0.59 & 1.11 & 24.8 & $\mathrm{C}$ & -0.0 & +1.0 & 3 & [5] \\
\hline$*_{1413}$ & $\ldots$ & 053648.6 & -692443 & 1.00 & 0.78 & $\mathrm{C}$ & $\mathrm{C}$ & -0.4 & - & 1 & HD $37974(\mathrm{Be}, \mathrm{LBV})$ \\
\hline$*^{*} 1425$ & $\ldots$ & $\begin{array}{l}053707.6 \\
0538.7\end{array}$ & $\begin{array}{l}-693127 \\
-6927\end{array}$ & $\begin{array}{l}0.07: \\
0.38:\end{array}$ & $\begin{array}{l}1.33 \\
0.85\end{array}$ & 2.1: & $\begin{array}{l}\mathrm{C} \\
\mathrm{C}\end{array}$ & $\begin{array}{l}+1.0 \\
+0.1\end{array}$ & -0.2 & $\begin{array}{l}1 \\
2\end{array}$ & $\begin{array}{l}\text { NGC } 2055 \\
{[5]}\end{array}$ \\
\hline 1467 & $\ldots$ & 053855 & -6901 & 0.93 & 2.77: & $\mathrm{C}$ & $\mathrm{C}$ & +0.2 & - & 2 & HD $269926(\mathrm{WN}, \mathrm{V}=13.1)$ \\
\hline 1483 & $\ldots$ & 053921.3 & -693052 & 0.74 & $\mathrm{C}$ & $\mathrm{C}$ & $\mathrm{C}$ & - & - & 2 & HD 269923 (B6Iab), Brey 91 (WN) \\
\hline${ }^{*} 1519$ & & 054033.5 & -690054 & $0.19:$ & 0.56 & $\mathrm{C}$ & $\mathrm{C}$ & +0.2 & - & 2 & BI 264 (B1II) \\
\hline${ }^{*} 1522$ & $\ldots$ & 054036.7 & -692414 & 0.85 & 0.78 & $\mathrm{C}$ & $\mathrm{C}$ & -0.4 & - & 1 & HD 38489 (Be) \\
\hline 1523 & $\ldots$ & 054040 & -6951 & 0.56 & 1.66 & $\mathrm{C}$ & $\mathrm{C}$ & +0.2 & - & 2 & HII region \\
\hline${ }^{*} 1582$ & $\ldots$ & 054232.1 & -691423 & 0.37 & & $\mathrm{C}$ & $\mathrm{C}$ & $<-0.5$ & - & 1 & LH $111, \mathrm{OB}$ assoc. \\
\hline${ }^{*} 1622$ & $\ldots$ & 054352.0 & -692605 & 0.37 : & 1.11 & 12.4: & $\mathrm{C}$ & $+0.2:$ & $+0.7:$ & 3 & {$[5]$} \\
\hline${ }^{*} 1678$ & $\ldots$ & 054557.0 & .671535 & $0.07:$ & 1.66 & 1.2 & $\mathrm{C}$ & +1.1 & -0.5 & 1 & SK - 67266 (O8Iab) \\
\hline$*_{1763}$ & ... & 055130.1 & -710345 & 0.15 & $0.11:$ & & $\mathrm{C}$ & -0.5 & $<+0.2$ & 1 & HD 270190 (A3); NGC 2134 \\
\hline
\end{tabular}

Notes to Table 7 : [], references as defined in Table 2; see also section 4.2. LI-LMC 1130: Zijlstra et al. (paper II) find a bright R counterpart (SP 44-29), and Reid et al. find 2 possible optical identifications; the infrared colours indicate that the star is not obscured; either the star is not associated with the IRAS source (Zijlstra et al.), either it is a hot star, or it is embedded in a nebula. 
Table 8. Comparison between the IRAS flux determinations

\begin{tabular}{|c|c|c|c|c|c|c|c|c|c|c|c|c|c|c|}
\hline \multirow[t]{2}{*}{ LI } & \multirow{2}{*}{ TRM } & \multirow{2}{*}{ IRAS-PSC } & \multicolumn{3}{|c|}{$s_{12}$} & \multicolumn{3}{|c|}{$S_{25}$} & \multicolumn{3}{|c|}{$S_{60}$} & \multicolumn{3}{|c|}{$S_{100}$} \\
\hline & & & LI & TRM & PSC & LI & TRM & PSC & LI & TRM & PSC & LI & TRM & PSC \\
\hline 375 & 117 & $05039-6724$ & 0.37 & 0.30 & 0.71 & 1.22 & 0.69 & 1.96 & $\mathrm{C}$ & - & $<34.3$ & $\mathrm{C}$ & - & $<83.6$ \\
\hline 383 & 048 & $05042-6720$ & 0.56 & 0.58 & 0.54 & 0.56 & 0.27 & 0.24 & 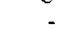 & 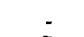 & $<34.3$ & - & - & $<83.6$ \\
\hline 416 & 134 & $05054-6739$ & & & $<0.51$ & 0.33 & 0.18 & 0.11 & . & $\mathrm{C}$ & $<3.6$ & 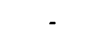 & $\mathrm{C}$ & $<19.2$ \\
\hline $\begin{array}{l}710 \\
463\end{array}$ & 009 & $05073-6752$ & 0.22 & 0.15 & $<0.25$ & 0.44 & 0.30 & 0.35 & $0.8:$ & - & $<3.2$ & - & - & $<18.1$ \\
\hline 513 & 012 & $05094-6751$ & $0.15:$ & 0.13 & $<0.25$ & 0.44 & 0.42 & 0.44 & & & $<2.4$ & - & 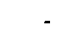 & $<12.8$ \\
\hline 528 & 023 & $05099-6740$ & 0.19 & 0.18 & $<0.25$ & 0.44 & 0.41 & 0.44 & 0.8 & 0.38 & 0.7 & 4.2 & . & $<19.0$ \\
\hline 532 & 091 & $05100-6629$ & 0.78 & 0.93 & 0.99 & 0.22 & 0.19 & 0.23 & - & - & $<0.4$ & & . & $<9.8$ \\
\hline 564 & 140 & $05110-6711$ & $0.07:$ & 0.19 & $<0.25$ & 0.28 & & 0.27 & $\mathrm{C}$ & - & $<5.8$ & $\mathrm{C}$ & - & $<35.2$ \\
\hline 567 & 100 & $05110-6616$ & 0.19 & 0.17 & $<0.27$ & 0.33 & 0.30 & 0.28 & - & - & $<0.40$ & & - & $<10.9$ \\
\hline 570 & 004 & $05112-6755$ & 0.41 & 0.42 & 0.48 & 0.33 & 0.29 & 0.34 & 1.2 & - & 0.91 & 2.1: & - & $<21.8$ \\
\hline 571 & 024 & $05113-6739$ & 0.33 & 0.26 & 0.30 & 0.17 & 0.17 & $<0.25$ & 1.2 & - & $<2.3$ & & & $<16.9$ \\
\hline 578 & 072 & & 0.19 & 0.14 & & $0.11:$ & & & $\mathrm{C}$ & & & $\mathrm{C}$ & . & \\
\hline 612 & 043 & $0.5128-6723$ & 0.26 & 0.36 & 0.37 & 0.33 & 0.31 & 0.33 & & 0.3 & $<2.6$ & & . & $<15.2$ \\
\hline 612 & $\begin{array}{l}450 \\
036\end{array}$ & $05148-6730$ & $\begin{array}{l}0.26 \\
0.26\end{array}$ & $\begin{array}{l}0.30 \\
0.34\end{array}$ & 0.42 & 0.44 & 0.23 & 0.26 & $0.8:$ & - & $<2.3$ & $\mathrm{C}$ & 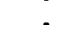 & $<72.0$ \\
\hline 678 & 110 & $05154-6535$ & 4.07 & 4.07 & 5.31 & 2.00 & 1.69 & 1.80 & $1.2:$ & & 0.49 & - & . & $<1.3$ \\
\hline 775 & 028 & & 0.22 & 0.21 & & 0.11 : & - & & 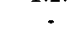 & - & . & - & . & \\
\hline 793 & 020 & $05190-6748$ & 0.30 & 0.34 & 0.37 & 0.22 & 0.23 & 0.28 &. & $\ldots$ & $<2.4$ & - & - & $<13.4$ \\
\hline 800 & 008 & 05193.6754 & 0.30 & 0.38 & 0.30 & 0.11 : & - & $<0.25$ & 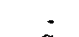 & - & $<2.7$ & & - & $<25.5$ \\
\hline 812 & 006 & $\ldots$ & 0.15 & 0.19 & & 0.11 & - & 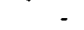 & $\mathrm{C}$ & - & - & $\mathrm{C}$ & 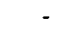 & - \\
\hline 814 & 090 & & 0.26 & 0.14 & & & & & & 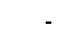 & 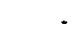 & & 政 & \\
\hline 855 & 014 & $05214-6749$ & 0.41 & 0.23 & 0.24 & 2.66 & 1.88 & 1.60 & 6.2 & 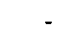 & $<32.9$ & 10.4: & - & $<27.6$ \\
\hline 861 & 011 & $05216-6753$ & 3.22 & 3.90 & 4.10 & 13.43 & 12.39 & 14.56 & 26.1 & 31.7 & $<32.9$ & 20.8 : & - & $<77.8$ \\
\hline 914 & $\begin{array}{l}011 \\
059\end{array}$ & & 0.37 & $\begin{array}{l}.90 \\
0.12\end{array}$ & & 0.33 & 0.11 & & $\mathrm{C}$ & $\mathrm{C}$ & & $\mathrm{C}$ & . & \\
\hline 932 & 108 & $05235-6544$ & 0.41 & 0.32 & 0.30 & 0.22 & 0.18 & 0.19 & $0.8:$ & - & $<1.6$ & 8.3: & . & $<35.2$ \\
\hline 938 & 001 & $05237-6755$ & 0.15 & 0.15 & 0.23 & 1.66 & 1.16 & 1.30 & $4.1:$ & 4.9 & $<27.2$ & $\mathrm{C}$ & - & $<19.7$ \\
\hline $\begin{array}{l}900 \\
957\end{array}$ & 016 & $05242-6748$ & 0.19 & 0.18 & $<0.25$ & 0.22 & 0.26 & 0.34 & 7.1. & 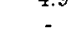 & $<2.6$ & - & - & $<17.7$ \\
\hline 1055 & 040 & & 0.19 & 0.16 & & 0.11 & & & $\mathrm{C}$ & 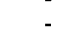 & & $\mathrm{C}$ & - & $<11.8$ \\
\hline 1082 & 095 & $05273-6624$ & 0.30 & 0.25 & 0.29 & 0.44 & 0.29 & 0.37 & . & : & $<2.0$ & & : & $<16.2$ \\
\hline 1107 & 065 & $\ldots$ & 0.19 & 0.15 & - & 0.11 : & & - & $0.4:$ & - & - & 2.1: & - & - \\
\hline 1116 & 114 & & 0.15 & 0.12 & & 0.22 & 0.18 & & $\mathrm{C}$ & 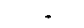 & & $\mathrm{C}$ & - & \\
\hline 1130 & 099 & $05289-6617$ & 0.11 & 0.13 & $<0.26$ & 0.44 & 0.37 & 0.43 & - & - & $<1.9$ & . & - & $<32.5$ \\
\hline 1135 & 085 & $05291-6643$ & 0.30 & 0.32 & 0.35 & 0.17 & 0.10 & $<0.25$ & - & - & $<2.0$ & - & . & $<19.1$ \\
\hline 1149 & 054 & $05293-6715$ & 0.41 & 0.27 & 0.32 & 0.33 & 0.22 & 0.18 & . & 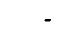 & $<3.9$ & - & . & $<23.6$ \\
\hline 1155 & 069 & & 0.19 & 0.19 & & 0.22 & 0.15 & & . & . & & ـ & & \\
\hline 1170 & 049 & $05299-6720$ & 0.19 & 0.18 & 0.21 & 0.22 & 0.12 & $<0.25$ & - & . & $<1.7$ & - & - & $<23.2$ \\
\hline 1177 & 079 & $05300-6651$ & $0.26:$ & 0.22 & 0.30 & 0.22 & 0.15 & $<0.25$ & . & . & $<1.3$ & - & - & $<8.3$ \\
\hline 1186 & 075 & $05303-6655$ & 0.22 & 0.19 & 0.25 & 0.22 & 0.12 & $<0.25$ & - & : & $<5.0$ & - & . & $<29.6$ \\
\hline 1190 & 046 & $05304-6722$ & 0.48 & 0.42 & 0.48 & 0.22 & 0.24 & 0.25 & . & $=$ & $<3.4$ & . & - & $<23.0$ \\
\hline 1234 & $\begin{array}{l}080 \\
089\end{array}$ & $05315-6631$ & 0.33 & 0.26 & 0.28 & 0.22 : & 0.21 & 0.19 & - & - & $<2.6$ & . & - & $<135$. \\
\hline 1238 & 101 & $05316-6604$ & 0.41 & 0.36 & 0.40 & 0.44 & 0.35 & 0.38 & : & $=$ & $<2.2$ & - & - & $<13.6$ \\
\hline 1241 & 087 & & 0.11 & 0.16 & & $0.17:$ & 0.12 & & & 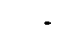 & & -5 & - & \\
\hline 1259 & 112 & $05321-6744$ & 1.48 & 0.82 & 0.98 & 5.99 & & 5.82 & $\mathrm{C}$ & . & $<58.5$ & C & - & $<192$. \\
\hline 1266 & 104 & $05324-6551$ & 4.2 & 5.54 & 5.12 & 1.55 & 1.17 & 1.52 & - & 0.2 & $<1.9$ & - & - & $<13.6$ \\
\hline 1280 & 058 & & 0.52 & 0.30 & & 0.44 & 0.31 & & & & & - & - & \\
\hline 1281 & 005 & $05327-6757$ & 0.41 & 0.53 & 0.70 & 0.56 & 0.44 & 0.55 & - & . & $<3.2$ & . & - & $<22,2$ \\
\hline 1286 & 060 & $05329-6708$ & 0.85 & 0.92 & 0.98 & 1.83 & 1.59 & 1.48 & 0.4 & . & $<2.7$ & - & - & $<27.6$ \\
\hline 1292 & 021 & $05330-6743$ & 1.18 & 0.72 & 0.74 & 4.22 & 3.23 & 3.18 & $\mathrm{C}$ & . & $<58.5$ & $\mathrm{C}$ & - & 91.7 \\
\hline $\begin{array}{l}1292 \\
1294\end{array}$ & $\begin{array}{l}021 \\
078\end{array}$ & $\begin{array}{l}05330-614.3 \\
05331-6650\end{array}$ & $\begin{array}{l}1.88 \\
0.26\end{array}$ & 0.27 & $\begin{array}{l}.1 .4 \\
0.25\end{array}$ & $\begin{array}{l}4.22 \\
0.11:\end{array}$ & 0.19 & $\begin{array}{l}3.10 \\
0.12\end{array}$ & . & $\cdot$ & $<2.5$ & . & - & $<12.1$ \\
\hline 1304 & 063 & $05334-6706$ & 0.37 & 0.3 & 0.30 & 0.22 & 0.18 & 0.21 & - & 0.30 & $<3.4$ & - & . & $<27.9$ \\
\hline $\begin{array}{l}1304 \\
1315\end{array}$ & $\begin{array}{l}003 \\
039\end{array}$ & UDS34-0/00 & 0.19 & 0.14 & 0.50 & 0.3 & $\begin{array}{l}.10 \\
0.09\end{array}$ & 0.21 & $\bar{C}$ & $\mathrm{C}$ & & $\dot{C}$ & $\dot{C}$ & \\
\hline 1317 & 149 & $05338-6617$ & & & $<0.25$ & 0.1 & 0.15 & 0.18 & - & - & $<2.5$ & . & - & $<11.9$ \\
\hline 1360 & 062 & & 0.19 & 0.12 & & 0 . & 0.13 & & & 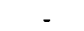 & & - & - & \\
\hline 1366 & 068 & $05354-6657$ & 0.33 & 0.30 & 0.33 & 0.2 & 0.22 & 0.29 & & - & $<2.8$ & - & . & $<23.5$ \\
\hline 1382 & 077 & $05360-6648$ & 0.22 & 0.22 & 0.24 & 0.22 & 0.15 & $<0.25$ & $0.8:$ & & $<2.3$ & 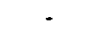 & - & $<13.1$ \\
\hline 1386 & 145 & & 0.26 & & & 0.22 & 0.10 & & 5.0 & 2.3 & & C & - & \\
\hline 1395 & 098 & $05363-6619$ & 1.59 & 1.84 & 1.84 & 0.44 & 0.43 & 0.51 & & - & $<3.5$ & - & - & $<72.5$ \\
\hline 1399 & 067 & $05364-6657$ & 0.33 & 0.32 & 0.35 & 0.33 & 0.27 & 0.34 & . & - & $<3.3$ & - & - & $<19.3$ \\
\hline 1513 & 148 & $05404-6619$ & & - & $<0.25$ & 0.44 & 0.33 & 0.41 & - & . & $<2.6$ & - & . & $<13.8$ \\
\hline 1587 & 055 & & 0.15 & 0.16 & & & & & & & & & & \\
\hline 1602 & 135 & 05 & 0.1 & & $<0.25$ & 0.22 & 0.12 & 0.10 & 0.8 & . & $<3.5$ & C & & $<27.0$ \\
\hline 1623 & 019 & 743 & 8.18 & 10.66 & 12.22 & 4.55 & 4.55 & 4.57 & 0.8 & - & $<1.5$ & $\mathrm{C}$ & - & $<21.4$ \\
\hline
\end{tabular}

